\title{
Superconductivity in ultrasmall metallic grains ${ }^{\text {a }}$
}

\author{
Jan von Delft \\ Institut für Theoretische Festkörperphysik \\ Universität Karlsruhe \\ 76128 Karlsruhe \\ Germany \\ vondelft@th.physik.uni-bonn.de \\ Received 01.08.2000, accepted 22.08.2000 by ue
}

\begin{abstract}
We review recent experimental and theoretical work on superconductivity in ultrasmall metallic grains, i.e. grains sufficiently small that the conduction electron energy spectrum becomes discrete. The discrete excitation spectrum of an individual grain can be measured by the technique of single-electron tunneling spectroscopy, and reveals parity effects indicative of pairing correlations in the grain. After introducing the discrete BCS model that has been used to model such grains, we review a phenomenological, grand-canonical, variational BCS theory describing the paramagnetic breakdown of these pairing correlations with increasing magnetic field. We also review recent canonical theories that have been developed to describe how pairing correlations change during the crossover, with decreasing grain size, from the bulk limit to the limit of few electrons, and compare their results to those obtained using Richardson's exact solution of the discrete BCS model.
\end{abstract}

Keywords: superconductivity, metallic grains

PACS: $74.20 . \mathrm{Fg}, 74.20 .-\mathrm{z}, 74.25 . \mathrm{Ha}, 74.80 . \mathrm{Fp}$

\section{Contents}

\begin{tabular}{lll}
\hline & Introduction & 3
\end{tabular}

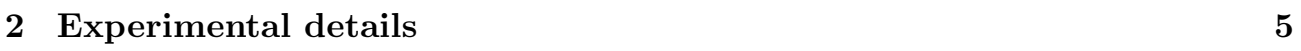

$\begin{array}{lll}3 \text { A gap in the excitation spectrum } & 10\end{array}$

$4 \quad$ A discrete BCS model for ultrasmall grains with pairing correlations 11

4.1 A simple reduced BCS interaction plus a Zeeman term . . . . . . . . . 11

4.2 Why orbital diamagnetism is negligible in ultrasmall grains . . . . . . 13

4.3 Choice of numerical values for model parameters . . . . . . . . . . . . 14

4.4 Some general properties of the eigenstates - the blocking effect . . . . 15

\begin{tabular}{|lll}
5 & Canonical characterization of pairing correlations & 17
\end{tabular}

5.1 The grand-canonical BCS wavefunction . . . . . . . . . . . 18

5.2 Canonically meaningful definition for the pairing parameter . . . . . . 18

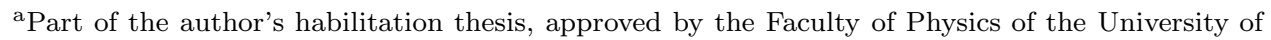
Karlsruhe, July 2000.
} 
5.3 Redistribution of occupation probability across $\varepsilon_{\mathrm{F}} \ldots \ldots \ldots 20$

5.4 Gauge symmetry breaking . . . . . . . . . . . . . . . . . 21

5.5 Making contact with standard BCS theory . . . . . . . . . . . . . 22

$\begin{array}{lll}6 & \text { Generalized variational BCS approach } & 23\end{array}$

6.1 The generalized variational Ansatz . . . . . . . . . . . . . . . 24

6.2 General numerical solution - illustration of the blocking effect . . . . . 26

\begin{tabular}{|lll}
7 & Softening of the $H$-induced transition to a paramagnetic state & 29
\end{tabular}

8 Excitation spectrum in a magnetic field 32

9 Measurable consequences of the blocking effect: parity effects 35

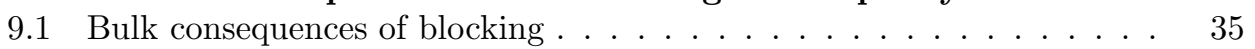

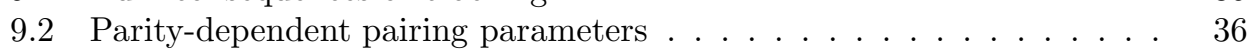

9.3 Matveev-Larkin parity parameter . . . . . . . . . . . . . . . . . 36

9.4 Parity effect for pairbreaking energies . . . . . . . . . . . . 38

\begin{tabular}{|ll}
\hline 10 Richardson's exact solution & 40
\end{tabular}

10.1 General eigenstates . . . . . . . . . . . . . . . . . . . 40 40

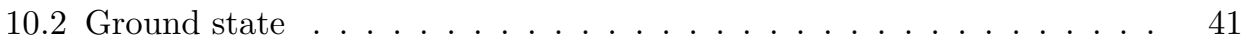

10.3 General comment . . . . . . . . . . . . . . . . . . . . 42

11 Comparison of other canonical methods with the exact solution 42

12 Qualitative differences between the bulk and the few-electron regimes 45

\begin{tabular}{ll}
\hline 13 Effect of level statistics & 48
\end{tabular}

$\begin{array}{ll}14 \text { Finite temperature parity effects } & 49\end{array}$

14.1 Parity-projected mean-field theory ... . . . . . . . . . . . . . . . . . 49

14.2 Variational extensions of BCS theory . . . . . . . . . . . . . . 51

14.3 Static path approximation . . . . . . . . . . . . . . . . . 51

14.4 Re-entrant spin susceptibility . . . . . . . . . . . . . . . . . 54

\begin{tabular}{ll}
\hline 15 Summary and outlook & 55
\end{tabular} 


\section{Introduction}

Since its discovery by Kammerlingh Onnes in 1911, superconductivity has become one of the most-studied phenomena in condensed matter physics, and its microscopic explanation via the highly successful pairing theory proposed in 1957 by Bardeen, Cooper and Schrieffer (BCS) [1] is one of the landmark achievements of 20th century physics. Yet, despite the long history of the subject, to this day experimental advances in sample fabrication and measurement techniques continue to reveal novel aspects of superconductivity, which often require extensions or modifications of the existing theoretical framework.

The subject of this review, superconductivity in ultrasmall metallic grains, is a case in point: In the mid 1990's, Ralph, Black and Tinkham (RBT) succeeded for the first time to directly measure the discrete excitation spectrum of individual ultrasmall metallic grains (of radii $r \lesssim 5 \mathrm{~nm}$ and mean level spacings $d \gtrsim 0.1 \mathrm{meV}$ ) using a technique called single-electron-tunneling spectroscopy: by attaching such a grain via oxide tunnel barriers to two leads they constructed a single-electron transistor having the grain as central island, and showed that a well-resolved, discrete excitation spectrum could indeed be extracted from the conductance [2]-[7].

This opened up a new frontier in the study of electron correlations in metals, since the ability to resolve discrete energy levels allows the nature of electron correlations to be studied in unprecedented detail. Since 1995, RBT's technique has been used to probe superconducting pairing correlations in Al grains [3, 7, nonequilibrium excitations [7]-[10] and spin-orbit interactions [2, 4, 11, 12, 13 in normal grains, and ferromagnetic correlations in Co grains 14, 15. A comprehensive survey of all experimental and theoretical developments (up to March 2000) relating to spectroscopic studies of ultrasmall metallic grains may be found in the review of von Delft and Ralph [16]. The present review is an excerpt of Ref. [16], and is devoted exclusively to superconductivity (more precisely, to pairing correlations) in ultrasmall metallic grains.

For several reasons, RBT's experiments on superconducting pairing correlations in ultrasmall $\mathrm{Al}$ grains attracted quite some attention [17]-40]:

First, for largish $(r \gtrsim 5 \mathrm{~nm})$ Al grains, RBT's measurements revealed a rather striking parity effect [3, 7, 17]: a grain with an even number of electrons had a distinct spectroscopic gap $(\gg d)$ but an odd grain did not. This is clear evidence for the presence of superconducting pairing correlations in these grains, and indicates that a BCS-like theory would be appropriate for their description.

Second, the spectroscopic gap for even grains was driven to zero by an applied magnetic field, hence the paramagnetic breakdown of pairing correlations could be studied in detail 18, 19.

Third, even the "largish" grains were so small that standard grand-canonical BCS mean field theory is no longer applicable: (a) the single-particle mean level spacing $d=1 / \mathcal{N}\left(\varepsilon_{F}\right) \sim 1 / \mathrm{Vol}$ [where $\mathcal{N}(\varepsilon)$ is the density of states per spin species] was comparable to the bulk superconducting gap, which we shall denote by $\tilde{\Delta}$, so that a mean field approach is no longer reliable (it requires $d \ll \tilde{\Delta}$ ); and (b), the number of electrons on such a grain is well-defined, hence a canonical theory is required. RBT's experiments stimulated the development of corresponding extensions of BCS theory.

Fourth, in RBT's smallest grains $(r \lesssim 3 \mathrm{~nm})$, the distinct spectroscopic gap ob- 
served for largish even grains could no longer be unambiguously discerned. This observation revived an old but fundamental question: What is the lower size limit for the existence of superconductivity in small grains?: Anderson had addressed this question already in 1959 [41, arguing that if the sample is so small that its electronic eigenspectrum becomes discrete, "superconductivity would no longer be possible" when its mean level spacing $d$ becomes larger than the bulk gap $\tilde{\Delta}$. Heuristically, this is obvious (see Fig. 7 below): $\tilde{\Delta} / d$ is the number of free-electron states that pair-correlate (those with energies within $\tilde{\Delta}$ of $\varepsilon_{\mathrm{F}}$ ), i.e. the "number of Cooper pairs" in the system; when this becomes $\lesssim 1$, it clearly no longer makes sense to call the system "superconducting".

Although Anderson's answer is correct in general, it generates further questions: What, precisely, does "superconductivity" mean in ultrasmall grains, for which many of the standard criteria such as zero resistivity, Meissner effect and Josephson effect, are not relevant $\mathrm{A}$ ? What happens in the regime $d \gtrsim \tilde{\Delta}$ in which superconductivity has broken down? Is the breakdown parity dependent? How is it influenced by a magnetic field? This review attempts to provide detailed answers to these and related questions.

The review is divided into two distinct parts. Part I (Secs. 2 to 9) is devoted to RBT's experiments and their detailed theoretical interpretation. After briefly discussing the experimental setup and summarizing the main experimental results, we analyze and qualitatively explain the latter in the framework of a phenomenological theory by Braun et al. [18, 19, 42]. This theory offers a simple intuitive picture for visualizing the pairing correlations and how these change when the grain size is decreased.

Part II (Secs. 10 to 14) is devoted to further theoretical developments, inspired by RBT's experiments but not directly concerned with their interpretation [17]- 40 . In particular, RBT's new experiments stimulated a number of theoretical attempts to quantitatively describe the crossover from the bulk limit $d \ll \tilde{\Delta}$, where superconductivity is well-developed, to the fluctuation-dominated regime of $d \gg \tilde{\Delta}$, where pairing correlations survive only in the form of weak fluctuations. Describing this crossover constituted a conceptual challenge, since the standard grand-canonical mean-field BCS treatment of pairing correlations [17]-[24] breaks down for $d \gtrsim \tilde{\Delta}$. This challenge elicited a series of increasingly sophisticated canonical treatments of pairing correlations 29- 37], based on a simple reduced BCS Hamiltonian for discrete energy levels, which showed that the crossover is completely smooth, but, interestingly, depends on the parity of the number of electrons on the grain [17]. Very recently, the main conclusions of these works were confirmed [35] using an exact solution of the discrete-level BCS model, discovered by Richardson in the context of nuclear physics in the 1960s [43- 51]. (The existence of this solution came as a surprise - in the form of a polite letter from its inventor - to those involved with ultrasmall grains, since hitherto it had apparently completely escaped the attention of the condensed-matter community.)

A detailed outline of the contents of the two parts may be found in the opening paragraph of each, or in the table of contents.

${ }^{\mathrm{a}}$ For an isolated nm-scale grain, (i) its resistivity is not defined, since electron motion is ballistic and the mean free path is boundary-limited; (ii) the grain radius is smaller than the penetration depth, so that no Meissner effect occurs; and (iii) the electron number is fixed, so that the order parameter cannot have a well-defined phase. 


\section{Part I: Experiment and phenomenological theory}

Part I of this review is devoted to RBT's experiments and their detailed theoretical interpretation. It is organized as follows: section

(2) is devoted to experimental details;

(3) summarizes RBT's main experimental results;

(4) proposes a phenomenological model for an isolated ultrasmall grain;

(5) discusses how pairing correlations can be visualized in a fixed- $N$ system and explains when and in what sense it can be called "superconducting";

(6) presents a generalized variational BCS approach for calculating the eigenenergies of various variational eigenstates of general spin $s$;

(7) discusses how an increasing magnetic field induces a transition from a paircorrelated state to a normal paramagnetic state;

(8) presents theoretical tunneling spectra of the RBT type, which are in qualitative agreement with RBT's measurements;

(9) discusses various parity effects that are expected to occur in ultrasmall grains.

\section{$2 \quad$ Experimental details}

In the first generation of RBT's experiments of 1995 [2, 3, 4], a grain made from $\mathrm{Al}$ (a superconducting material) was connected to two metal leads via high-resistance tunnel junctions, with capacitances $C_{\mathrm{L}}$ and $C_{\mathrm{R}}$, say. In the next generation of 1997 [7], the grain was also coupled capacatively to a gate, with capacitance $C_{\mathrm{g}}$. The resulting device, schematically depicted in Fig. 1 (a), has the structure of a SET, with the grain as central island. The circuit diagram for an SET is shown in Fig. 1(b). Applying a bias voltage $V$ between the two leads causes a tunnel current $I$ to flow between the leads through the grain, via incoherent sequential tunneling through the tunnel junctions. The current can be influenced by changing the gate voltage $V_{\mathrm{g}}$ (hence the name "transistor"), which tunes the electrostatic potential on the grain and thereby also its average number of electrons $N$. (For devices without a gate these two quantities cannot be tuned and instead have some sample-dependent, fixed value. For such devices, set $C_{\mathrm{g}}=0$ in all formulas below.)
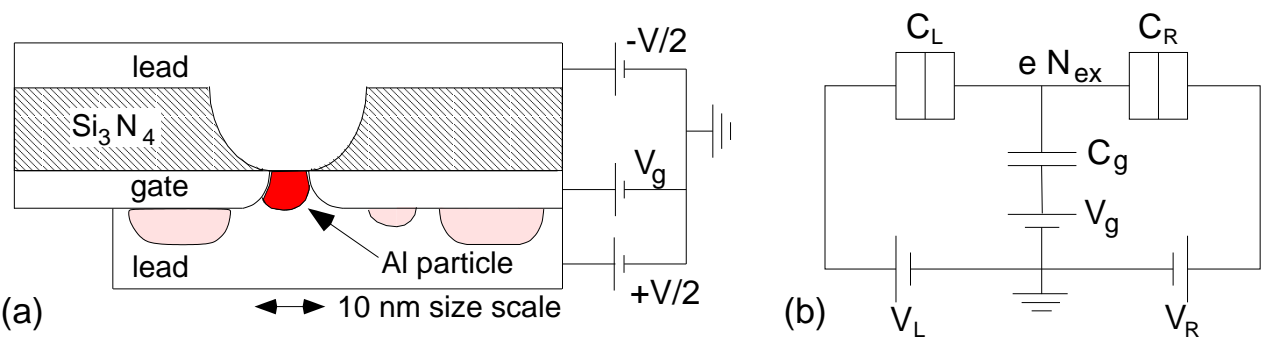

Fig. 1 (a) Schematic cross section of the ultrasmall SETs studied by RBT in [], and (b) the corresponding circuit diagram. 
The physics of SETs had been clarified in the early 1990s [52 through extensive studies of lithographically defined SETs of mesoscopic size, i.e. with micron-scale central islands. The fundamentally new aspect of RBT's work was that their SETs, made by a novel fabrication technique (described in Ref. [2]), were nanoscopic in size: they had ultrasmall grains with radii between $15 \mathrm{~nm}$ and $2 \mathrm{~nm}$ as central islands, which were thus several orders of magnitude smaller in volume than in previous experiments. This had two important consequences:

1. The grain's charging energy $E_{\mathrm{C}} \equiv e^{2} / 2 C$ was much larger than for mesoscopic $S E T s$, ranging roughly between 5 and $50 \mathrm{meV}$ (where $C \equiv C_{\mathrm{L}}+C_{\mathrm{R}}+C_{\mathrm{g}}$ ). $E_{\mathrm{C}}$ is the scale that determines the energy cost for changing the electron number $N$ by one. Since for ultrasmall grains it far exceeds all other typical energy scales of the SET, such as those set by the bias voltage $(V \lesssim 1 \mathrm{mV})$, the temperature $(T \lesssim 4.2 \mathrm{~K})$ and the bulk superconducting gap for $\mathrm{Al}\left(\Delta_{\text {bulk }}=0.18 \mathrm{meV}\right)$, fluctuations in electron number are strongly suppressed.

2. Discrete eigenstates of the conduction electron energy spectrum became resolvable - their mean level spacing $d$ ranged from 0.02 to $0.3 \mathrm{meV}$. This agrees in order of magnitude with the estimate $d=1 / \mathcal{N}\left(\varepsilon_{\mathrm{F}}\right)$ obtained using the free-electron expression for the density of states $\mathcal{N}\left(\varepsilon_{\mathrm{F}}\right)$ at the Fermi surface of a 3D grain,

$$
d=\frac{2 \pi^{2} \hbar^{2}}{m k_{\mathrm{F}} \mathrm{Vol}}=\frac{1.50 \mathrm{eV} \cdot \mathrm{nm}^{2}}{k_{\mathrm{F}} \mathrm{Vol}}
$$

where, for example, $k_{\mathrm{F}}=17.5 \mathrm{~nm}^{-1}$ for Al. The measured $d$-values are much larger than $k_{\mathrm{B}} T$ for the lowest temperatures attained (around $T \simeq 30 \mathrm{mK}$ ), but on the order of $\Delta_{\text {bulk }}$. However, the number of conduction electrons for grains of this size is still rather large (between $10^{4}$ and $10^{5}$ ).

Since the two scales $E_{\mathrm{C}}$ and $d$ differ by at least an order of magnitude, they manifest themselves in two distinct and easily separable ways in the low-temperature $I$ - $V$ curves of RBT's devices:

1. Coulomb-blockade phenomena: When $V$ is varied on a large scale of tens of $\mathrm{mV}$ for fixed $V_{\mathrm{g}}$ [Fig. 2], the $I-V$ curves have a typical "Coulomb-staircase form" characteristic of SETs: zero current at low $|V|$ (the "Coulomb blockade" regime), sloping steps equally spaced in $V$, and step thresholds sensitive to $V_{\mathrm{g}}$. This proves that the tunnel current flows only through one grain. The maximal width of the flat step of zero current around $|e V|=0$ is governed, in order of magnitude, by $E_{\mathrm{C}}$ and typically varies between 5 and $50 \mathrm{mV}$.

As $V_{g}$ is varied, the $I-V$ curves periodically repeat. The "orthodox theory" for Coulomb blockade phenomena [54, 55, 56, 16] explains this as follows: the electrostatic work required to add $N_{\text {ex }}$ excess electrons with a total charge of $e N_{\text {ex }}($ with $e<0$ ) to a grain with initial random off-set charge $Q_{0}$, while the time-independent voltages $V_{\mathrm{L}}, V_{\mathrm{R}}$ and $V_{\mathrm{g}}$ of the left and right leads and the gate electrode, respectively, are held fixed, has the form

$$
E_{\text {pot }}\left(N_{\text {ex }}\right)=e V_{\mathrm{D}} N_{\mathrm{ex}}+E_{\mathrm{C}} N_{\mathrm{ex}}^{2} .
$$




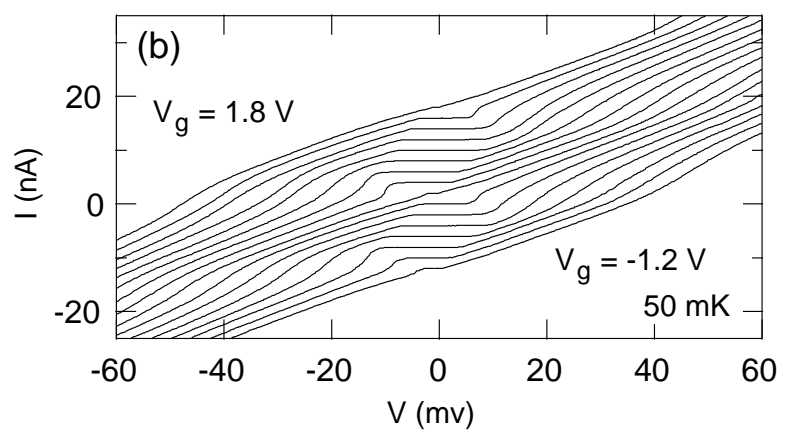

Fig. 2 Current-voltage curves for an ultrasmall SET [1] at $50 \mathrm{mK}$, artificially offset on the vertical axis for a set of equally-spaced values of $V_{\mathrm{g}}$ between -1.2 and $1.8 \mathrm{~V}$. The $I$ - $V$ curves display Coulomb-staircase structure on a bias voltage scale of tens of $\mathrm{mV}$. By fitting these to standard SET theory [53, the SET's basic parameters can be determined: $R_{\mathrm{L}}=3.5 \mathrm{M} \Omega$, $R_{\mathrm{R}}=0.2 \mathrm{M} \Omega, C_{\mathrm{L}}=3.5 \mathrm{aF}, C_{\mathrm{R}}=9.4 \mathrm{aF}, C_{\mathrm{g}}=0.09 \mathrm{aF}, E_{\mathrm{C}}=46 \mathrm{meV}$. Assuming the grain shape to be hemispherical and using the measured capacitances to estimate its surface areas (see [1, 16 for details), the grain's radius and mean level spacing were estimated as $r \simeq 4.5 \mathrm{~nm}$ and $d \simeq 0.45 \mathrm{meV}$.

Here $V_{\mathrm{D}} \equiv\left(Q_{0}+\sum_{r=\mathrm{L}, \mathrm{R}, \mathrm{g}} C_{r} V_{r}\right) / C$ represents the electrostatic potential on the grain, and $E_{\mathrm{C}} N_{\mathrm{ex}}^{2}$ represents the Coulomb interaction energy of the $N_{\text {ex }}$ excess electrons due to their mutual repulsion. Since, for given $V_{\mathrm{g}}$, the system adjusts $N_{\mathrm{ex}}$ such as to minimize $E_{\text {pot }}\left(N_{\mathrm{ex}}\right)$, the $I-V$ characteristics are $V_{\mathrm{g}}$-periodic, with period $e / C_{\mathrm{g}}$.

2. Fine-structure due to level discreteness: When $V$ is varied on the much smaller scale of a few $\mathrm{mV}$ around the threshold of the Coulomb blockade regime and the temperature is sufficiently low $(T \ll d)$, the $I$ - $V$ curves have a step-like substructure, and correspondingly, the differential conductance $(\mathrm{d} I / \mathrm{d} V)$ curves contain a series of fine peaks, see Fig. 3. As first pointed out by Averin and Korotkov [57], such small steps in the $I-V$ curve are expected to occur whenever the voltage drop across one of the tunnel junctions (say $r=\mathrm{L}, \mathrm{R}$ ) equals the threshold energy at which the rate for tunneling across that junction into or out of one of the grain's discrete energy eigenstates becomes nonzero, since this opens up another channel for carrying current across that junction. Such tunneling processes are ilustrated schematically in Fig. A.

More formally, let $\Sigma_{\alpha_{N} \alpha_{N^{\prime}}^{\prime}}^{r \pm}$ denote the rate for the tunneling transition $\left|\alpha^{\prime}\right\rangle_{N^{\prime}} \rightarrow$ $|\alpha\rangle_{N}$ between two grain eigenstates (with electron number $N^{\prime}$ and $N=N^{\prime} \pm 1$, and eigenenergies $\mathcal{E}_{\alpha^{\prime}}^{N^{\prime}}, \mathcal{E}_{\alpha}^{N}$, respectively,), induced by transferring an electron across barrier $r$ onto (upper sign) or off (lower sign) the grain. A golden rule calculation (see Sec. 2.3.3 of Ref. [16] for details) then shows that these rates have the form

$$
\Sigma_{\alpha \alpha^{\prime}}^{r \pm}=f\left(\mathcal{E}_{\alpha}^{N}-\mathcal{E}_{\alpha^{\prime}}^{N^{\prime}} \mp e\left(V_{r}-V_{\mathrm{D}}\right)\right) \Gamma_{\alpha \alpha^{\prime}}^{r \pm},
$$

where $f(E)=1 /\left(e^{E / k_{\mathrm{B}} T}+1\right)$ is the Fermi function, $V_{r}-V_{\mathrm{D}}$ is the voltage drop (electrostatic potential difference) between lead $r$ and the grain, and $\Gamma_{\alpha \alpha^{\prime}}^{r \pm}$ are transition probabilities. With increasing transport voltage $V=V_{\mathrm{L}}-V_{\mathrm{R}}$, a current step thus occurs each time a "bottleneck rate" $\Sigma_{\alpha \alpha^{\prime}}^{r \pm}$ (associated with a rate-limiting tunneling 


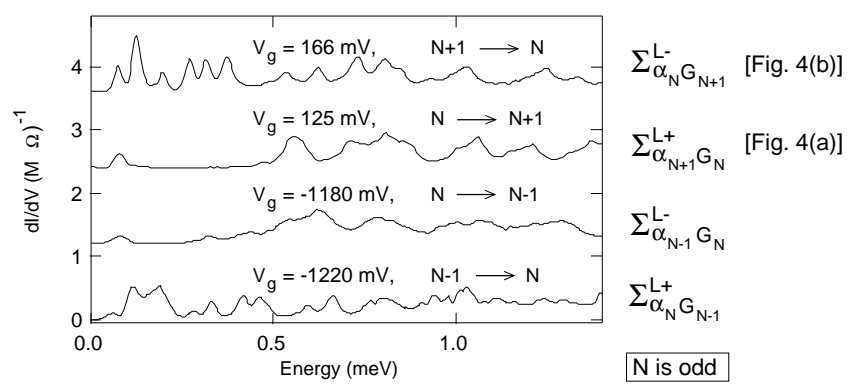

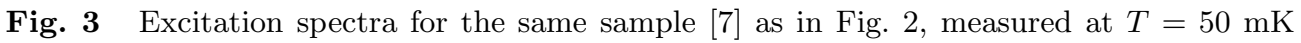
and $H=0.05 \mathrm{~T}$ (to drive the $\mathrm{Al}$ leads normal), for four different $V_{\mathrm{g}}$-values, corresponding to different values for the grain's average electron number (from top to bottom: $N+1, N, N, N-$ 1 ). The curves are artificially offset on the vertical axis and each is labeled by the associated bottleneck tunneling rate $\Sigma_{\alpha_{N} \mathrm{G}_{N^{\prime}}}^{r \pm}$ (from initial state $|\mathrm{G}\rangle_{N^{\prime}}$ to final state $|\alpha\rangle_{N}$, two of which are illustrated schematically in Fig. (1), the bottleneck barrier being $r=\mathrm{L}$ in this case. Plotted is $\mathrm{d} I / \mathrm{d} V$ vs. energy, where the latter is given by the voltage-to-energy conversion factor $|e V|\left(C_{\mathrm{R}}+C_{\mathrm{g}} / 2\right) / C=0.73|e V|$, which reflects the voltage drop across barrier $\mathrm{L}$ (for a derivation of this factor, see Sec. 2.3 of Ref. [16]). The sizeable spectroscopic gap between the first two peaks in the middle two curves, and its absence in the top and bottom curves, reflects the pairbreaking energy cost in the excitation spectrum of a superconducting grain with an even number of electrons, and implies that $N$ is odd.

process) is switched from "off" (exponentially small) to "on" (of order $\Gamma_{\alpha \alpha^{\prime}}^{r \pm}$ ), i.e. each time $e\left(V_{r}-V_{\mathrm{D}}\right)$ passes through a threshold at which one of the inequalities

$$
\pm e\left(V_{r}-V_{\mathrm{D}}\right) \geq \mathcal{E}_{\alpha}^{N}-\mathcal{E}_{\alpha^{\prime}}^{N^{\prime}}
$$

becomes true. The tunneling spectrum yields particularly useful information if the initial state for all bottleneck tunneling processes is always the ground state, $\left|\alpha^{\prime}\right\rangle_{N^{\prime}}=$ $|\mathrm{G}\rangle_{N^{\prime}}$ (whose electron number $N^{\prime}$ is determined by $V_{\mathrm{g}}$ ). This will be the case if the following conditions are met: (i) for the given (fixed) value of gate voltage $V_{\mathrm{g}}$, all bottleneck processes involve the same barrier; (ii) the temperature is sufficiently low $(T \ll d)$; and (iii) nonequilibrium effects are negligible (requiring relaxation rates on the grain to be much greater than tunneling rates). Under these conditions, which are satisfied by the data of Fig. 3 (and Fig. 5 below), the distances between the current steps or conductance peaks directly reflect the so-called fixed- $N$ excitation spectrum of the grain, i.e. the set of energy differences

$$
\delta \mathcal{E}_{\alpha \bar{\alpha}}^{N}=\mathcal{E}_{\alpha}^{N}-\mathcal{E}_{\bar{\alpha}}^{N}
$$

between the eigenenergies $\mathcal{E}_{\alpha}^{N}$ of all those $N$-electron eigenstates $|\alpha\rangle_{N}$ that are accessible final states from the initial ground state $|\mathrm{G}\rangle_{N^{\prime}}$, via a tunneling processes onto the grain if $N=N^{\prime}+1$, or off the grain if $N=N^{\prime}-1$.

For devices having a gate, two very interesting options exist: Firstly, by tuning $V_{\mathrm{g}}$

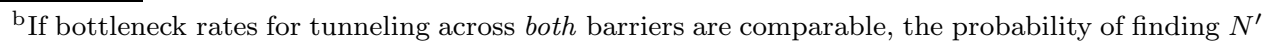
and $N$ electrons on the grain will be comparable, and the analysis is considerably more complicated; see Sec. 2.3.4 of 16 for details.
} 


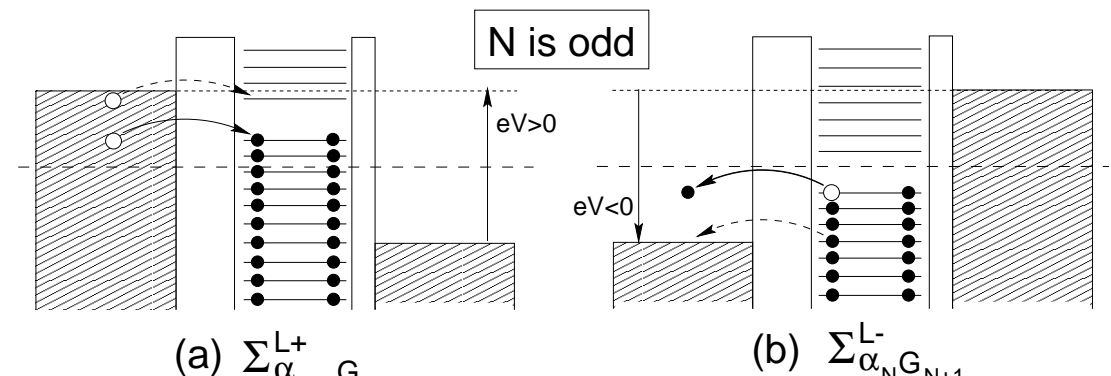

Fig. 4 Schematic depiction of bottleneck tunneling processes governing the excitation spectra of Fig. 3, for $N$ being odd, with rates: (a) $\Sigma_{\alpha_{N+1} G_{N}}^{L+}$ and (b) $\Sigma_{\alpha_{N} G_{N+1}}^{L-}$, corresponding to two different choices of $V_{\mathrm{g}}$ just below or above the degeneracy point at which the $N$ - and $(N+1)$-electron ground states are degenerate. The long-dashed line indicates the equilibrium, $V=0$ chemical potential of the $\mathrm{L}$ and $\mathrm{R}$ leads. Solid (dashed) arrows depict bottleneck tunneling transitions into the lowest- (highest) energy final states accessible for the chosen value of $V$, and filled circles represent the electron configuration of the lowest-energy final state. (For a more detailed discussion of such diagrams, in particular how they change with $V_{\mathrm{g}}$, see Sec. 2 of Ref. [16].)

such that the Coulomb blockade regime is large or small, so that the $V$-threshold at which current begins to flow is large or small, nonequilibrium effects can be maximized or minimized, respectively, depending on whether one chooses to study them or not. In this review, we consider only the near-equilibrium situation. Strong nonequilibrium was studied in Refs. [7]- $[10$ and is reviewed in Sec. 6 of Ref. [16]. Secondly, by tuning $V_{\mathrm{g}}$ by an amount large enough $\left(\simeq E_{\mathrm{C}} / e\right)$ to change $N^{\prime}$ by one unit, the influence on the spectrum of the parity of the number of electrons on the grain can be studied.

Parity effects occur, for instance, in the magnetic-field dependence of the fixed$N$ excitation spectrum, which can be obtained by simply tracing the motion of the conductance peak positions as a magnetic field is turned on (at fixed $V_{\mathrm{g}}$ ). This is shown in Fig. 5 below, which nicely illustrates the breaking of Kramers degeneracy by the applied field: For $H=0$, the grain will have time-reversal symmetry. For an even- $N$ grain, the many-electron wave function for the ground state will be a spin singlet, in order that the orbital energy be minimized. In contrast, the ground state of an odd- $N$ grain for $H=0$ necessarily is two-fold degenerate, by Kramers' theorem, forming a Kramers doublet. When $H$ is turned on, this doublet is Zeeman-split by $\pm \frac{1}{2} \mu_{\mathrm{B}} g H$. Therefore, for an even- $N$ grain at small $H$, the lowest-lying tunneling excitations correspond to transitions from the even-grain ground state singlet to the odd-grain ground state doublet, i.e. to two states split by $H$, so that the lowest- $V$ conductance peak will exhibit Zeeman splitting in an applied field [Fig. E(b)]. On the other hand, for an odd- $N$ grain with $T \ll \mu_{\mathrm{B}} g H / k_{\mathrm{B}}$, the odd-grain ground state will be the lower-energy state of the Kramers doublet; the lowest-lying tunneling excitation will thus consist only of a single transition from this odd-grain ground state to the even-grain ground state singlet, so that the lowest- $V$ conductance peak will not split

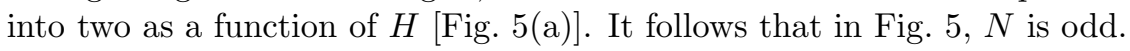




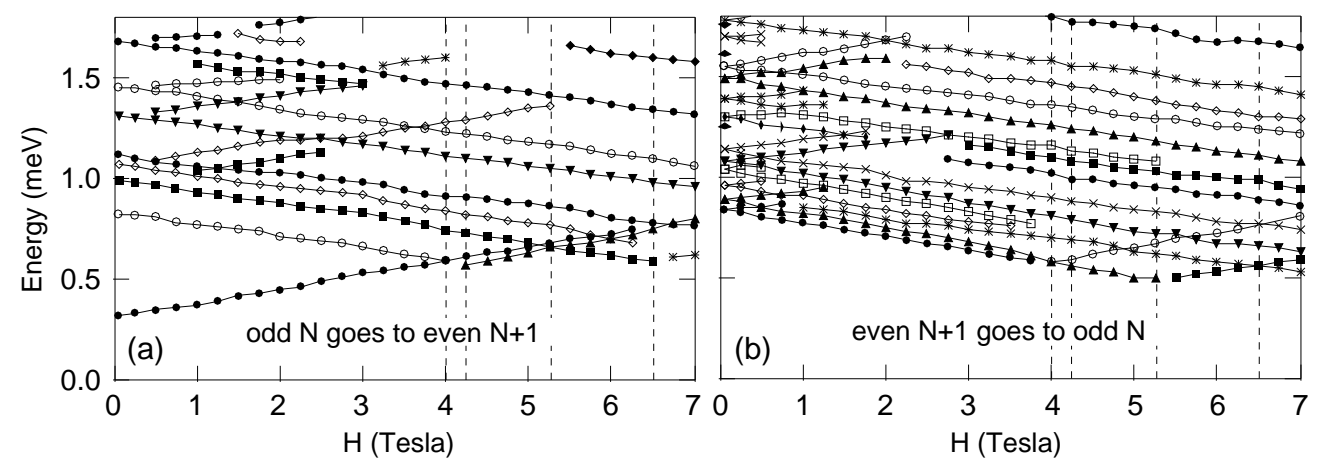

Fig. 5 Magnetic field dependence 7] of excitation spectra such as those of Fig. 3 and taken for the same grain, at (a) $V_{\mathrm{g}} \approx 110 \mathrm{mV}$ and (b) $V_{\mathrm{g}} \simeq 180 \mathrm{mV}$ [corresponding to the second and topmost curves of Fig. 3, respectively]. Each line represents a distinct conductance peak in the $\mathrm{d} I / \mathrm{d} V$ curve and traces how its energy changes with $H$. Upward-moving peaks are broader and less distinct than downward-moving ones (for reasons poorly understood) and can be followed only for a limited range of $H$ before they are lost in the background. The distances between lines directly give the grain's (a) fixed- $(N+1)$ and (b) fixed- $N$ excitation spectrum, where $N$ is odd; the corresponding bottleneck processes are schematically illustrated in Figs. 4(a) and (b), respectively. The vertical dashed lines indicate the first four level-crossing fields $H_{s, s^{\prime}}$ (defined in Eq. (41) and assigned by comparison with Fig. 12, see Sec. 8), namely $H_{0,1}=4 \mathrm{~T}, H_{1 / 2,3 / 2}=4.25 \mathrm{~T}, H_{1,2}=5.25 \mathrm{~T}$ and $H_{3 / 2,5 / 2}=6.5 \mathrm{~T}$ with uncertainty $\pm 0.13 \mathrm{~T}$ (half the $H$-resolution of $0.25 \mathrm{~T}$ ).

\section{$3 \quad$ A gap in the excitation spectrum}

The spectra shown in Figs. 3 and 5, which are typical for RBT's results for largish Al grains $(r \gtrsim 5 \mathrm{~nm})$, reveal a very striking feature: if the number of electrons on the grain in the final state after the bottleneck tunneling process is even (middle two curves of Fig. 3), the excitation spectra display a spectroscopic gap between the first two conductance peaks that is significantly larger than the mean spacing between subsequent peaks, whereas no such gap occurs if the final-state electron number is odd (top and bottom curves of Fig. 33). In other words, even- $N$ excitation spectra (number parity $p=0)$ are gapped, whereas odd- $N$ excitation spectra $(p=1)$ are not. This is even more clearly apparent when comparing Figs. 5(a) and (b), which give the magnetic-field $(H)$ dependence of an even- $N$ and odd- $N$ excitation spectrum, respectively. However, in their smallest grains $(r \lesssim 3 \mathrm{~nm})$ no such clear paritydependent spectroscopic gap could be discerned.

BRT interpreted these observations as evidence for superconducting pairing correlations in their larger grains, using notions from the BCS theory of superconductivity: in an even grain, all excited states involve at least one broken Cooper pair, i.e. two BCS quasiparticles, and hence lie at least $2 \Delta$ above the fully-paired BCS ground state; in contrast, in an odd grain all states have at least one unpaired electron, i.e. at least one quasiparticle, and hence no significant gap exists between ground- and excited states. Fig. 1 is a cartoon illustration of this interpretation, if one imagines two electrons drawn on the same energy level to represent a "Cooper pair" (making this 
cartoon precise will be one of the main goals of review): in Fig. 1. (a) the final electron number is even, and all final excited states (reached via dashed arrows) have one less "Cooper pair" than the final ground state (reached via the solid arrow); in contrast, in Figs. $4(\mathrm{~b})$ the final electron number is odd, and the final ground and excited states have the same number of "Cooper pairs".

The approximately linear $H$-dependence of the excitation spectra in Fig. 同 was attributed by RBT to the Zeeman energy shifts of discrete levels in a magnetic field (its effect on orbital motion is neglible, as will be discussed in Sec. 1.2). The fact that the lowest state in Figs. 同(a) or (b) does not or does display Zeeman splitting, respectively, implies that $N$ is odd, as mentioned above. The reduction of the spectroscopic gap in Fig. 5(a) therefore is purely due to Zeeman energy shifts and has nothing to do with the reduction of the BCS gap parameter due to pair-breaking that occurs in bulk samples in a magnetic field [58. A detailed discussion of the spectra's magnetic field dependence will be given in Secs. 7 and 8 .

For completeness, it should be remarked that a spectral gap in ultrasmall superconducting grains was observed as long ago as 1968 by Giaever and Zeller [59, 60], who studied tunneling through granular thin films containing electrically insulated Sn grains. They found gaps for grain sizes right down to the critical size estimated by Anderson (radii of $2.5 \mathrm{~nm}$ in this case), but were unable to prove that smaller particles are always "normal". RBT's experiments are similar in spirit to this pioneering work, but their ability to focus on individual grains makes a much more detailed study possible.

\section{A discrete BCS model for ultrasmall grains with pairing correlations}

In this section we construct a model for an isolated ultrasmall grain with pairing correlations, using phenomenological arguments valid for the regime $d \lesssim \tilde{\Delta}$. The model, which we shall call "discrete BCS model", allows a rather detailed qualitative understanding of the measurements of RBT [18, 19] and hence is "phenomenologically successful". For $d \gg \tilde{\Delta}$ it is unrealistically simple, however, and should rather be viewed as a toy model for studying how pairing correlations change as the grain is made smaller and smaller.

\subsection{A simple reduced BCS interaction plus a Zeeman term}

Following the philosophy of the "orthodox theory" for Coulomb blockade phenomena [54, 16], we assume that the only effect of the Coulomb interaction is to contribute an amount $E_{\mathrm{C}} N_{\mathrm{ex}}^{2}$ [cf. Eq. (2)] to the eigenenergy of each eigenstate of the grain. Since the charging energy is huge ( 5 to $50 \mathrm{meV}$ ) in ultrasmall grains, this term strongly suppresses number fluctuations around the optimal value of $N_{\mathrm{ex}}$, so that, to an excellent approximation, all energy eigenstates will also be number eigenstates. 9 Since $E_{\mathrm{C}} N_{\mathrm{ex}}^{2}$ is constant within every fixed- $N$ Hilbert space, we shall henceforth ignore it, with the understanding that the model we are about to construct should always be solved in

\footnotetext{
c An exception occurs at a so-called degeneracy point, where $E_{\text {pot }}\left(N_{\text {ex }}\right)=E_{\text {pot }}\left(N_{\text {ex }}+1\right)$; the resulting complications will not be considered here.
} 
a fixed- $N$ Hilbert space (and that the use of grand-canonical approaches below, after having dropped $E_{\mathrm{C}} N_{\mathrm{ex}}^{2}$, simply serves as a first approximation to the desired canonical solution).

The only symmetry expected to hold in realistic, irregularly-shaped ultrasmall grains at zero magnetic field is time-reversal symmetry. We therefore adopt a singleparticle basis of pairs of time-reversed states $|j \pm\rangle$, enumerated by a discrete index $j$. Their discrete energies $\varepsilon_{j}$ are assumed to already incorporate the effects of impurity scattering and the average of electron-electron interactions, etc. As simplest conceivable model that incorporates pairing interactions and a Zeeman coupling to a magnetic field, we adopt a Hamiltonian $\hat{H}=\hat{H}_{0}+\hat{H}_{\text {red }}$ of the following reduced BCS form:

$$
\hat{H}_{0}=\sum_{j, \sigma= \pm}\left(\varepsilon_{j}-\mu-\sigma h\right) c_{j \sigma}^{\dagger} c_{j \sigma}, \quad \hat{H}_{\mathrm{red}}=-\lambda d \sum_{i j} c_{i+}^{\dagger} c_{i-}^{\dagger} c_{j-} c_{j+} .
$$

Here $-\sigma h \equiv \sigma \frac{1}{2} \mu_{B} g H$ is the Zeeman energy of a spin $\sigma$ electron in a magnetic field $H$, and we shall take $h>0$ below. Models of this kind had previously been studied by Strongin et al. [61], Mühlschlegel et al. [62, 63] and Kawataba 664, 65]. The first application to RBT's grains for $h=0$ was by von Delft et al. 117 and for $h \neq 0$ by Braun et al. 17, 18, 19].

Due to level repulsion the $\varepsilon_{j}$ 's will, to first approximation, be uniformly spaced. Unless otherwise specified, we shall for simplicity always (except in Sec. 13) take a completely uniform spectrum with level spacing $d$. Fluctuations in the level spacings have been studied with methods of random matrix theory [20], with qualitatively similar results (see Sec. 13). For a system with a total of $N$ electrons, where the electron number parity $p \equiv N \bmod 2$ is equal to 0 for even $N$ and 1 for odd $N$, we use the label $j=0$ for the lowest-lying non-doubly-occupied level (with occupation number $p$ ) in the $T=0$ Fermi sea, which we shall denote by $\left|\mathrm{F}_{N}\right\rangle$. We choose the Fermi energy at $\varepsilon_{\mathrm{F}} \equiv 0$ writed

$$
\varepsilon_{j}=j d+(1-p) d / 2,
$$

thereby taking the doubly-occupied and empty levels of $\left|\mathrm{F}_{N}\right\rangle$ to lie symmetrically above and below $\varepsilon_{\mathrm{F}}$ (see e.g. Fig. 8 below) The parameter $\mu$ in Eq. (5) is, in g.c. theories, the chemical potential, whose valued determines the average particle number. For canonical theories, which make no reference to a chemical potential, $\mu$ is not needed and can be dropped (i.e. set equal to 0 ).

The pairing interaction is of the reduced BCS form, in that it scatters a pair of electrons from one pair of time-reversed states into another. . It is taken to include only states whose energy separation from the Fermi energy lies within the cutoff given by the Debye frequency: $\left|\varepsilon_{j}\right|<\omega_{\mathrm{D}}$. The pair-coupling constant in Eq. (6) is written as

\footnotetext{
$\mathrm{d}$ This convention differs slightly from that used in 17, 18, 19, namely $\varepsilon_{j}=j d+\varepsilon_{0}$. The latter is a little less convenient, resulting, e.g., in a $p$-dependent chemical potential for the variational BCS ground states discussed below, $\mu_{p}^{\mathrm{BCS}}=\varepsilon_{0}+(p-1) d / 2$, whereas (7) results simply in $\mu_{p}^{\mathrm{BCS}}=0$.

e Note that the use of a reduced BCS interaction means that couplings between non-time-reversed pairs of states are neglected. A theoretical motivation, based on random matrix theory, for this reduced form may be found in Ref. [10], or in Sec. 6.13 of Ref. [16]. Experimental evidence for the sufficiency of the reduced form is discussed in Refs. 18, 19] and, in most detail, in Sec. 4.7 of Ref. [16.
} 
$\lambda d$, where $\lambda$ is a dimensionless parameter independent of the grain's volume, to make it explicit that both $\hat{H}_{0}$ and $\hat{H}_{\text {red }}$ make extensive $(\propto \mathrm{Vol})$ contributions to the ground state energy (since the number of terms in each sum $\sum_{j}$ in Eq. (6) scales with $N$, and $\left.d \propto \varepsilon_{\mathrm{F}} / N\right)$. The "bulk gap" of the model, obtained by solving the standard BCS gap equation [Eq. (27)] at $T=0$ in the bulk limit, thus is

$$
\tilde{\Delta}=\omega_{\mathrm{D}} / \sinh (1 / \lambda)
$$

To be precise, by "bulk limit" we shall always mean $d / \tilde{\Delta} \rightarrow 0$ and $N \rightarrow \infty$ while the product $N d$ is kept fixed, and use $d \sum_{j} \rightarrow \int \mathrm{d} \varepsilon_{j}$.

An applied magnetic field will completely penetrate an ultrasmall grain, since its radius (typically $r \lesssim 5 \mathrm{~nm}$ ) is much smaller than the penetration length of $50 \mathrm{~nm}$ for bulk Al. The Zeeman term in Eq. (6) models the fact that RBT's measured tunnel spectra of Fig. 5 evolve approximately linearly as a function of magnetic field, with $g$ factors between 1.95 and 2 (determined from the differences between measured slopes of up- and downward-moving lines). Deviations from $g=2$ probably result from spinorbit scattering, known to be small but nonzero in thin Al films 66, 67, but neglected below (where $g=2$ is used).

Intuitively speaking, it is clear that the discrete BCS model introduced above contains all ingredients necessary to make contact with the spectra of Fig. 5: it is formulated in terms of discrete levels, it contains a pairing interaction which is known, from bulk BCS theory [1, 58, to cause a gap in the excitation spectrum, and it contains a Zeeman term that will cause eigenenergies to linearly depend on an applied magnetic field. Indeed, we shall see in Sec. 8 that it can be used to obtain a rather detailed qualitative understanding of the spectra of Fig. 5.

\subsection{Why orbital diamagnetism is negligible in ultrasmall grains}

Of course, a magnetic field in principle also couples to the orbital motion of the conduction electrons - in bulk samples, this is the origin of the Meissner effect. Orbital effects in spherical and cylindrical superconductors whose dimensions are smaller than the penetration depth were first considered by Larkin [68]. However, in grains as small as those of RBT, orbital diagmagnetic effects are negligible [11], just as for thin films in a parallel magnetic field 66, 67]. The reason is as follows:

Let $H_{\text {orb }}$ denote the field scale above which orbital diamagnetism becomes important. If, in a random-matrix description of the grain's spectrum, $H_{\text {orb }}$ is formally associated with the field at which the crossover between the symplectic and unitary ensembles, driven by the orbital effects of the magnetic field, is complete, it is found [69] that

$$
H_{\mathrm{orb}} \approx \frac{\Phi_{0}}{r^{2} \sqrt{E_{\text {Thouless }} / d}},
$$

\footnotetext{
f Claims of smaller $g$ factors made in Ref. [3 are wrong, the result of confusing different orbital states as Zeeman-split spin states. This was made clear in Ref. [f, where it was observed that upward-trending Zeeman states can have significantly smaller amplitude than downward-trending states, making them difficult to observe.
} 
where $E_{\text {Thouless }}$ is the Thouless energy and $\Phi_{0}\left(=h c / 2 e=2067 \mathrm{~T} \cdot \mathrm{nm}^{2}\right)$ is the flux quantum. An intuitive understanding for the origin of this result can be obtained by the following argument 70]: Associate $H_{\text {orb }}$ with the field at which the orbital splitting of the eigenenergies of two time-reversed states $|j \pm\rangle$ becomes comparable to the mean level spacing. Denoting the angular momentum of these states by $\pm\left\langle\hat{l}_{z}\right\rangle_{j} \hbar$, the orbital diamagnetic contribution to their eigenenergies is $\pm\left\langle\hat{l}_{z}\right\rangle_{j} \mu_{\mathrm{B}} H$, hence $H_{\text {orb }} \approx d /\left(2\left\langle\hat{l}_{z}\right\rangle_{j} \mu_{\mathrm{B}}\right)$. Now, the angular momentum of an electron traversing the "closed trajectory" corresponding to a discrete quantum level can be estimated as $\left\langle\hat{l}_{z}\right\rangle \hbar \approx m\left(A_{\mathrm{typ}} d / \hbar\right)$, where the bracketed factor is the typical (directed) area $A_{\text {typ }}$ covered by its trajectory divided by the period $\hbar / d$ of its motion. The number of bounces off the grain's boundaries during this time is roughly $E_{\text {Thouless }} / d$, since the $\hbar / E_{\text {Thouless }}$ is the time to cross the grain once. Hence the directed area is $A_{\text {typ }} \approx r^{2} \sqrt{E_{\text {Thouless }} / d}$, where the square root accounts for the fact that the direction of motion after each bounce is random [71]. Collecting the various estimates results in $H_{\text {orb }} \approx \Phi_{0} /\left(\pi r^{2} \sqrt{E_{\text {Thouless }} / d}\right)$, which, up to a factor of $\pi$, agrees with Eq. (9).

Now, assuming ballistic electron motion in the grain, the Thouless energy has the form $E_{\text {Thouless }} \approx \hbar v_{\mathrm{F}} /(a 2 r)$, where $a$ is a geometrical constant of order unity. Using $d$ from Eq. (1), we see that $H_{\text {orb }}$ grows like $r^{-3}$ with decreasing grain size. Taking $a=3$ (as in Ref. [12]), we find from Eq. (9) that hemispherical Al grains with radii of (say) $r \approx 3$ or $5 \mathrm{~nm}$ have $H_{\text {orb }} \approx 19$ or $7 \mathrm{~T}$, respectively. If larger values are used for $a$, as would be appropriate for more pancake-shaped grains [8], $H_{\text {orb }}$ would be even larger.

We may thus conclude that orbital diagmagnetic effects only begin to play a role for largish grains ( $\gtrsim 5 \mathrm{~nm}$ ), and then only for the highest fields (of $7 \mathrm{~T}$ ) studied by RBT. Indeed, some larger grains do show slight deviations from $H$-linearity [3] for large fields, which probably reflect the onset of such orbital effects; however, these are much smaller than Zeeman effects in the grains of present interest, and will be neglected here. Thus, the discrete BCS model assumes that Pauli paramagnetism due to the Zeeman energy completely dominates orbital diamagnetism, similarly to the case of thin films in parallel magnetic fields [66, 67.

\subsection{Choice of numerical values for model parameters}

When doing numerical calculations for the discrete BCS model, some choices must be made for the numerical values of its parameters (though slight changes in their values will not change the results qualitatively). We shall follow the choices made by Braun et al. [19], since these led to reasonable agreement between experimental and theoretical excitation spectra. For the Debye frequency they used the textbook value [72 for $\mathrm{Al}$ of $\omega_{\mathrm{D}}=34 \mathrm{meV}$. Making an appropriate choice for the "bulk gap" $\tilde{\Delta}$ is less straightforward, since its experimental value for systems of reduced dimensionality often differs from that of a truly bulk system, presumably due to (poorly-understood) changes in the phonon spectrum and the effective electron-phonon coupling. For example, for thin Al films [61, 73] it is known that $\tilde{\Delta}_{\text {thin film }} \simeq 0.38 \mathrm{meV}$, which is about twice as large as the gap of a truly bulk system, $\tilde{\Delta}_{\text {bulk }}=0.18 \mathrm{meV}$. (This increase in $\tilde{\Delta}$ is not universal, though; e.g., for $\mathrm{Nb} \tilde{\Delta}$ is smaller in thin films than in the bulk.) Since ultrasmall grains are in many ways analogous to thin films in a parallel magnetic field [see Sec. 7], Braun et al. adopted the thin-film value for grains too, i.e. 
used $\tilde{\Delta} \simeq 0.38 \mathrm{meV}$. These choices imply that the dimensionless pair-coupling constant $\lambda=\left[\sinh ^{-1}\left(\omega_{\mathrm{D}} / \tilde{\Delta}\right)\right]^{-1}$ [cf.Eq. (8)] has the value $\lambda=0.194$. (In Sec. 8 we shall see, a posteriori, that the choices $\tilde{\Delta}=0.34$ and $\lambda=0.189$ would have been slightly more appropriate.) Finally, for those numerical calculations that are explicitly cut-off dependent, Braun et al. smeared the cutoff of the BCS interaction over two singleelectron levels; this smooths out small discontinuities that would otherwise occur in $d$-dependent quantities each time the energy $\left|\varepsilon_{j}\right|$ of some large- $|j|$ level moves beyond the cutoff $\omega_{\mathrm{D}}$ when $d$ is increased.

Note that the above way of choosing $\lambda$ lumps into a single phenomenological constant all the poorly-understood effects of reduced dimensionality [61 on the phonons that mediate the attractive electron-electron interaction. Studying these effects in detail would be interesting in its own right, but would require systematic investigations with grains of well-controlled shapes and sizes. For the case of RBT's irregularlyshaped grains, using a phenomenological coupling constant seems the best one can do. Note, though, that the precise value of $\lambda$ is not very important as long as all energies are measured in units of $\tilde{\Delta}$ (as we shall do for all numerical calculations), since most of the $\lambda$-dependence is thereby normalized away. Therefore, the slight difference between the $\lambda$-values proposed above and those used in [31, 42, 35] (namely 0.224) hardly matters.

\subsection{Some general properties of the eigenstates - the blocking effect}

The eigenstates of the discrete BCS model of Eq. (6) have some simple but general properties that are worth stating at the outset.

Firstly, every eigenstate of $\hat{H}$ will also be an eigenstate of the number operator $\hat{N}=\sum_{j \sigma} c_{j \sigma}^{\dagger} c_{j \sigma}$, since $[\hat{H}, \hat{N}]=0$.

Secondly, since the interaction only involves levels within the cutoff energy $\omega_{\mathrm{D}}$ of $\varepsilon_{\mathrm{F}}$, the dynamics of those lying outside this range is trivial. We shall thus ignore them henceforth and focus only on the remaining set of interacting levels, denoting this set by $I$.

Thirdly, singly-occupied levels do not participate in the pair scattering described by $\hat{H}$ : "unpaired" electrons in such levels are not scattered to other levels, hence the labels of singly-occupied levels are good quantum numbers. Moreover, every unpaired electron Pauli-blocks the scattering of other pairs into its own singly-occupied level, i.e. it restricts the phase space available to pair scattering and thereby weakens the amount of pairing correlations, as we shall see in detail later. This was called the "blocking effect" by Soloviev [74], who discussed it extensively in the early 1960's in the context of nuclear physics. The eigenstates $|\alpha\rangle$ and corresponding eigenenergies $\mathcal{E}_{\alpha}$ of $\hat{H}$ thus have the following general forms:

$$
\begin{aligned}
|\alpha\rangle & =\left|\Psi_{n}, B\right\rangle=\prod_{i \in B} c_{i \sigma_{i}}^{\dagger}\left|\Psi_{n}\right\rangle \\
\left|\Psi_{n}\right\rangle & =\sum_{j_{1}, \ldots, j_{n}}^{U} \psi\left(j_{1}, \ldots, j_{n}\right) \prod_{\nu=1}^{n} b_{j_{\nu}}^{\dagger}|\mathrm{Vac}\rangle
\end{aligned}
$$




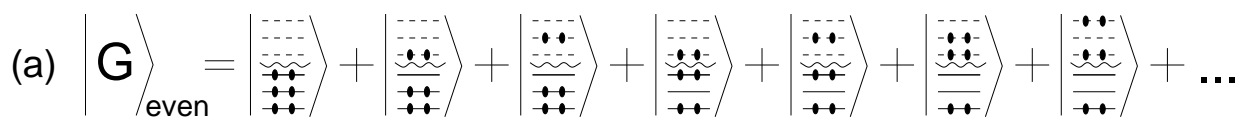

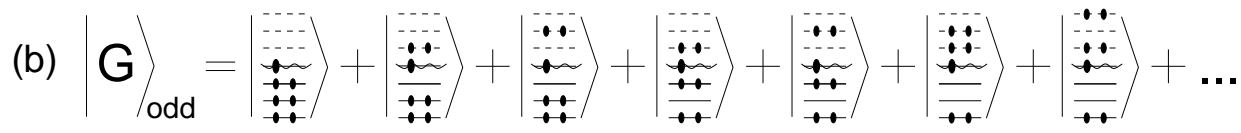

Fig. 6 A cartoon depiction of the exact ground state for a reduced BCS Hamiltonian, for $N$ being even (a) or odd (b): they are coherent superpositions of eigenstates of $\hat{H}_{0}$ (whose respective amplitudes are not depicted) that all have the same $N$; the leftmost of these is (a) the even or (b) the odd Fermi ground state $\left|F_{N}\right\rangle$, whose Fermi energy is indicated by the wavy line.

$$
\mathcal{E}_{\alpha}=\mathcal{E}_{n}+\mathcal{E}_{B}(h), \quad \mathcal{E}_{B}(h)=\sum_{i \in B}\left(\varepsilon_{i}-\mu-\sigma_{i} h\right)
$$

This describes $N=2 n+b$ electrons, $b$ of which are unpaired and sit in a set $B$ of singly-occupied, blocked levels, making a contribution $\mathcal{E}_{B}(h)$ to the total eigenenergy. The remaining $n$ pairs of electrons, created by the pair operators $b_{j}^{\dagger}=c_{j+}^{\dagger} c_{j-}^{\dagger}$, are distributed among the remaining set $U=I \backslash B$ of unblocked levels, with wavefunction $\psi\left(j_{1}, \ldots, j_{n}\right)\left(\sum_{j}^{U} \equiv \sum_{j \in I \backslash B}\right.$ denotes a sum over all unblocked levels in $\left.I\right)$. The corresponding state $\left|\Psi_{n}\right\rangle$ is an eigenstate of the pair number operator and a Hamiltonian $\hat{H}_{U}$ involving only pair operators:

$$
\begin{aligned}
& \sum_{j}^{U} b_{j}^{\dagger} b_{j}\left|\Psi_{n}\right\rangle=n\left|\Psi_{n}\right\rangle, \quad \hat{H}_{U}\left|\Psi_{n}\right\rangle=\mathcal{E}_{n}\left|\Psi_{n}\right\rangle \\
& \hat{H}_{U}=\sum_{i j}^{U}\left[2\left(\varepsilon_{j}-\mu\right) \delta_{i j}-\lambda d\right] b_{i}^{\dagger} b_{j} .
\end{aligned}
$$

Each eigenstate $\left|\Psi_{n}, B\right\rangle$ may be visualized as a coherent superposition of eigenstates of $\hat{H}_{0}$ that all lie in the same fixed- $N$ Hilbert space, and in all of which each pair of unblocked $(j \in U)$, time-reversed levels $|j \pm\rangle$ is either doubly occupied or empty. This is illustrated in Figs. 6(a) and (b), which schematically depict the exact ground states for even and odd $N$, respectively. The odd ground state has a single blocked level, at the Fermi energy, containing an unpaired electron. The latter somewhat weakens pairing correlations relative to the even ground state and hence leads to parity effects, which will be extensively discussed in later sections.

A useful measure for the amount of energy gained by $|\alpha\rangle$ via its correlations is its "condensation energy" relative to the uncorrelated state $|\alpha\rangle_{0}$,

$$
E_{\alpha}^{\text {cond }}=\mathcal{E}_{\alpha}-{ }_{0}\langle\alpha|\hat{H}| \alpha\rangle_{0}, \quad \text { where } \quad|\alpha\rangle_{0}=\prod_{i \in B} c_{i \sigma_{i}}^{\dagger}|U\rangle_{0}
$$

and $|U\rangle_{0}$ is the "Fermi ground state" in $U$, for which the $n$ pairs occupy the $n$ lowestlying levels in $U$. 
Note that $\hat{H}_{U}$ is $h$-independent, since the total Zeeman energy of any pair of electrons is zero. Hence the full $h$-dependence of the eigenenergies resides in the rather trivial contribution $\mathcal{E}_{B}(h)$ of the blocked levels, which is a very important and useful simplification.

Diagonalizing $\hat{H}_{U}$ would be trivial if the $b$ 's were true bosons. However, they are not, and in the subspace spanned by the set $U$ of all non-singly-occupied levels instead satisfy the "hard-core boson" relations,

$$
b_{j}^{\dagger 2}=0, \quad\left[b_{j}, b_{j^{\prime}}^{\dagger}\right]=\delta_{j j^{\prime}}\left(1-2 b_{j}^{\dagger} b_{j}\right), \quad\left[b_{j}^{\dagger} b_{j}, b_{j^{\prime}}^{\dagger}\right]=\delta_{j j^{\prime}} b_{j}^{\dagger},
$$

which reflect the Pauli principle for the fermions from which the $b$ 's are constructed. In particular, $b_{j}^{\dagger 2}=0$ implies that only those terms in (11) are non-zero for which the indices $j_{1}, \ldots j_{n}$ are all distinct.

The task of finding the eigenstates $\left|\Psi_{n}\right\rangle$ is thus highly non-trivial. Nevertheless, an exact solution does exist. Unbeknownst to most of the condensed-matter physics community, it was found and studied extensively by Richardson in the 1960's and will be presented in Sec. 10. Throughout the present part I, however, we shall use more well-known approaches based on the variational wavefunctions introduced by BCS [1], and that had been used to study the discrete BCS model before Richardson's solution was revived towards the end of 1998 .

\section{Canonical characterization of pairing correlations}

Since the discrete BCS model has the standard reduced BCS form, the most natural first step toward understanding its $T=0$ properties is to use BCS-like variational wavefunctions (or equivalently Bogoljubov's mean-field approach), and indeed this will be done in Secs. 6 to 9 . However, the limitations of such an approach should be realized at the outset: the spectra measured by RBT are excitation spectra for a grain with a fixed electron number $N$, and hence should be calculated for a grain with definite electron number $N$ (i.e. completely isolated from the rest of the world, e.g. by infinitely thick oxide barriers). In contrast, the variational wavefunctions of BCS [Eq. (17) below] do not have the fixed- $N$ form [Eq. (11)] which any true eigenstate should have, but instead are formulated in a grand-canonical (g.c.) framework (as is the Bogoljubov mean-field approach to which they are intimately related).

When considering a truly isolated superconductor such as a perfectly insulated grain (another example would be a superconductor levitating in a magnetic field due to the Meissner effect), one therefore needs to address the following question, which will be the main theme of the present section: how is one to incorporate the fixed- $N$ condition into BCS theory, and how important is it to do so? This issue is well understood and was discussed at length in the early days of BCS theory (Rickayzen's book gives a beautiful discussion [75), in particular in its application to pairing correlations in nuclei [76, p. 439] (see also the general remarks in [77). Nevertheless, for pedagogical reasons the arguments are well worth recapitulating in the present context.

We shall first remind the reader that the use of a g.c. framework is only a matter

of convenience, since the essence of the pairing correlations that lie at the heart of 
BCS theory is by no means inherently g.c. and can easily be formulated in canonical language 17. We then show how standard BCS theory fits into this scheme, point out that the differences between results obtained using g.c. and canonical wavefunctions are negligible for $d \ll \tilde{\Delta}$, and conclude that for the purposes of gaining a phenomenological understanding of the experimental data, standard grand-canonical BCS theory should be sufficient. Nevertheless, the fundamental question of how to improve on this theory, in order to achieve a truly canonical description and to properly treat fluctuation effects, which become important for $d \gtrsim \tilde{\Delta}$ [78, 79, 24, is interesting and important in its own right and will be addressed at length in Sec. 9.4.

For simplicity, throughout the present subsection 5 we shall consider only the even ground state in the thermodynamic limit (in which even-odd differences are negligible), so that $U=I$ and blocking effects need not be worried about.

\subsection{The grand-canonical BCS wavefunction}

Conventional BCS theory describes the pairing correlations induced by an attractive pairing interaction such as $\hat{H}_{\text {red }}$ of Eq. (6) within a g.c. ensemble, formulated on a Fock space of states in which the total particle number $N$ is not fixed. This is illustrated by BCS's famous variational ground state Ansatz

$$
|\mathrm{BCS}\rangle=\prod_{j}\left(u_{j}+\mathrm{e}^{\mathrm{i} \phi_{j}} v_{j} b_{j}^{\dagger}\right)|\mathrm{Vac}\rangle, \quad \text { with } \quad u_{j}^{2}+v_{j}^{2}=1,
$$

where the variational parameters $u_{j}$ and $v_{j}$ are real and $\phi_{j}$ is a phase (which, it turns out, must be $j$-independent, for reasons discussed below). |BCS $\rangle$ is not an eigenstate of $\hat{N}$ and its particle number is fixed only on the average by the condition $\langle\hat{N}\rangle_{\text {BCS }}=N$, which determines the g.c. chemical potential $\mu$. Likewise, the commonly used g.c. definition

$$
\Delta_{\mathrm{gc}} \equiv \lambda d \sum_{j}\left\langle b_{j}\right\rangle_{\mathrm{BCS}}=\lambda d \sum_{j} u_{j} v_{j} \mathrm{e}^{\mathrm{i} \phi_{j}}
$$

for the superconducting pairing parameter only makes sense in a g.c. ensemble, since $\left\langle b_{j}\right\rangle$ would trivially give zero when evaluated in a canonical ensemble, formulated on a strictly fixed- $N$ Hilbert space of states. (We shall use the term "pairing parameter" instead of "order parameter", since the latter carries the connotation of a phase transition, which would require the thermodynamic limit $N \rightarrow \infty$, which is not applicable for ultrasmall grains).

\subsection{Canonically meaningful definition for the pairing parameter}

A theory of strictly fixed- $N$ superconductivity must evidently entail modifications of conventional BCS theory. However, these are only of technical, not of conceptual nature, since the essence of the pairing correlations discovered by BCS can easily be formulated in a canonically meaningful way, including a definition for the pairing parameter. We shall now attempt to explain, in intuitive, non-technical terms, how this may be done (our discussion is indebted to that of Rickayzen [75]). Readers with a 
preference for rigor may consult Secs. 10 to 12 for a corroboration, using Richardson's exact solution, of the arguments presented below.

Let $|G\rangle$ be the exact even ground state of the system, depicted in Fig. 6(a). As explained in Sec. 4.4, it is a coherent superposition of eigenstates of $\hat{H}_{0}$ that all have the same $N$ and in all of which each pair of time-reversed levels $|j \pm\rangle$ is either doubly occupied or empty. Due to this coherent superposition, $|G\rangle$ entails strong pairing correlations, whose essential properties may be understood by investigating how they modify the correlators

$$
C_{i j} \equiv\left\langle b_{i}^{\dagger} b_{j}\right\rangle, \quad \bar{v}_{j}^{2} \equiv C_{j j}=\left\langle b_{j}^{\dagger} b_{j}\right\rangle, \quad \bar{u}_{j}^{2} \equiv\left\langle b_{j} b_{j}^{\dagger}\right\rangle,
$$

relative to the form these take on for the Fermi ground state $\left|\mathrm{F}_{N}\right\rangle$ :

$$
\left(C_{i j}\right)_{\mathrm{F}}=\delta_{i j}\left(\bar{v}_{j}^{2}\right)_{\mathrm{F}}, \quad\left(\bar{v}_{j}^{2}\right)_{\mathrm{F}}=\theta\left(-\varepsilon_{j}\right), \quad\left(\bar{u}_{j}^{2}\right)_{\mathrm{F}}=\theta\left(\varepsilon_{j}\right) .
$$

$C_{i j}\left(=C_{j i}^{*}\right)$ is the matrix element for the interaction to be able to scatter a pair of electrons from level $j$ to $i$, and $\bar{v}_{j}^{2}$ and $\bar{u}_{j}^{2}$ are the probabilities to find level $j$ doubly occupied or empty, respectively. The pairing correlations in $|G\rangle$ must be such that $\hat{H}_{\text {red }}$ lowers the ground state energy below that of the uncorrelated Fermi sea $\left|\mathrm{F}_{N}\right\rangle$ by an amount that is extensive $\left(\propto N \propto d^{-1}\right)$ in the thermodynamic limit. Clearly, this requires that $\left\langle\hat{H}_{\text {red }}\right\rangle_{\mathrm{G}}-\left\langle\hat{H}_{\text {red }}\right\rangle_{\mathrm{F}}$ is negative and extensive, i.e. that

$$
\left.\lambda d \sum_{i j}\left[C_{i j}-\left(C_{i j}\right)_{\mathrm{F}}\right] \simeq \lambda d \sum_{i} \sum_{j<i} 2 \operatorname{Re}\left(C_{i j}\right) \propto N \quad \text { (and positive }\right) .
$$

In the second expression we neglected the diagonal terms, since their number is so small $($ only $\propto N)$ that $\lambda d \sum_{j}\left[\bar{v}_{j}^{2}-\left(\bar{v}_{j}^{2}\right)_{\mathrm{F}}\right]$ is at best of order unity in the thermodynamic limit. For Eq. (21) to hold, $|\mathrm{G}\rangle$ must have two properties:

(i) the number of $C_{i j}$ 's that differ significantly from zero (i.e. are of order unity) should scale like $N^{2}$, i.e. one power of $N$ per index [75. p. 167];

(ii) most or all of the $C_{i j}$ for $i<j$ should have the same phase, since a sum over random phases would average out to zero.

Since a suitable pairing parameter should vanish in the thermodynamic limit unless both these conditions hold, the definition

$$
\Delta_{\text {can }}^{2} \equiv(\lambda d)^{2} \sum_{i j}\left(C_{i j}-\left\langle c_{i+}^{\dagger} c_{j+}\right\rangle\left\langle c_{i-}^{\dagger} c_{j-}\right\rangle\right)
$$

(or its square root) suggests itself, where the subscript emphasizes that (22) is meaningful in a canonical ensemble too, and we subtracted the "normal-state contribution

\footnotetext{
${ }^{\mathrm{g}}$ This subtraction was suggested to us by Moshe Schechter, who pointed out that then Eq. (22) has a natural generalization to position space: it is the spatial average, $\Delta_{\text {can }}^{2} \equiv(\lambda d)^{2} \int \mathrm{d} \vec{r}_{1} \mathrm{~d} \vec{r}_{2} \mathcal{F}\left(\overrightarrow{\vec{r}_{1}}, \vec{r}_{2}\right)$, of the two-point function

$$
\mathcal{F}\left(\vec{r}_{1}, \vec{r}_{2}\right) \equiv\left\langle\psi_{+}^{\dagger}\left(\vec{r}_{1}\right) \psi_{-}^{\dagger}\left(\vec{r}_{1}\right) \psi_{-}\left(\vec{r}_{2}\right) \psi_{+}\left(\vec{r}_{2}\right)\right\rangle-\left\langle\psi_{+}^{\dagger}\left(\vec{r}_{1}\right) \psi_{+}\left(\vec{r}_{2}\right)\right\rangle\left\langle\psi_{-}^{\dagger}\left(\vec{r}_{1}\right) \psi_{-}\left(\vec{r}_{2}\right)\right\rangle
$$

(with $\psi_{\sigma}(\vec{r}) \equiv \mathrm{Vol}^{-1 / 2} \sum_{\vec{k}} \mathrm{e}^{\mathrm{i} \vec{k} \cdot \vec{r}} c_{\vec{k} \sigma}$ ), which evidently measures the amplitude for the propagation of pairs as opposed to uncorrelated electrons. Other definitions for a canonically meaningful pairing parameter have been suggested [17, 31, 19, such as $\lambda d \sum_{j} \bar{u}_{j} \bar{v}_{j}$ or $\lambda d \sum_{j}\left[\left\langle b_{j}^{\dagger} b_{j}\right\rangle-\left\langle c_{j+}^{\dagger} c_{j+}\right\rangle\left\langle c_{j-}^{\dagger} c_{j-}\right\rangle\right]^{1 / 2}$, but these focus only on requirement (i) and fail to incorporate requirement (ii). A quantity very similar to Eq. 22 was recently proposed in Eq. (55) of Ref. [26], namely $(\lambda d)^{2} \sum_{i j}\left[C_{i j}-\left(C_{i j}\right)_{\lambda=0}\right]$.
} 
to $C_{i j}$." If (i) and (ii) hold, $\Delta_{\text {can }}$ will take on a finite value; its relation to a gap in the spectrum will become clear below. In the bulk limit, $\Delta_{\text {can }}$ can be shown [see Sec. 12] to reduce to the "bulk pairing parameter" $\tilde{\Delta}$ of Eq. (8).

\subsection{Redistribution of occupation probability across $\varepsilon_{\mathrm{F}}$}

Now, property (i) can be realized if all $C_{i j}$ in a finite ( $d$-independent) range of $\varepsilon_{i}$ 's and $\varepsilon_{j}$ 's around the Fermi energy $\varepsilon_{\mathrm{F}}$ differ significantly from zero; the width of this range will evidently determine the magnitude of $\Delta_{\text {can }}$ (provided (ii) also holds), which conversely can be viewed as a measure of this width. But a nonzero $C_{i j}$ evidently requires both $b_{i}^{\dagger} b_{j}|\mathrm{G}\rangle \neq 0$, implying $\left(\bar{v}_{j}\right)_{\mathrm{G}} \neq 0$ and $\left(\bar{u}_{i}\right)_{\mathrm{G}} \neq 0$, and also $\langle\mathrm{G}| b_{i}^{\dagger} b_{j} \neq 0$, implying $\left(\bar{v}_{i}\right)_{\mathrm{G}} \neq 0$ and $\left(\bar{u}_{j}\right)_{\mathrm{G}} \neq 0$. The product $\left(\bar{u}_{j} \bar{v}_{j}\right)_{\mathrm{G}}$ must thus be different from zero [in contrast to $\left(\bar{u}_{j} \bar{v}_{j}\right)_{\mathrm{F}}=0$ ] for all $\varepsilon_{j}$ within a finite range around $\varepsilon_{\mathrm{F}}$ (cf. Fig. 五). This can be achieved by smearing out the sharp steps of the $\theta$-functions of $\left(\bar{v}_{j}\right)_{\mathrm{F}}$ and $\left(\bar{u}_{j}\right)_{\mathrm{F}}$, so that $\left(\bar{v}_{j}\right)_{\mathrm{G}}\left[\mathrm{or}\left(\bar{u}_{j}\right)_{\mathrm{G}}\right]$ is nonzero also for a finite range of $\varepsilon_{j}$ above [or below] $\varepsilon_{\mathrm{F}}$. In other words, for $|\mathrm{G}\rangle$ some occupation probability must be redistributed (relative to $\left|\mathrm{F}_{N}\right\rangle$ ) from below to above $\varepsilon_{\mathrm{F}}$, as illustrated in Fig. 6. This redistribution, which was called pair-mixing in [17, 19, frees up phase space for pair scattering and so achieves a gain in interaction energy (provided (ii) also holds) that more than compensates for the kinetic energy cost incurred thereby.

Furthermore, note that properties (i) and (ii) imply, even without detailed calculations, that the spectrum will be gapped. Consider, for example, a "blocking excitation" that disrupts pairing correlations by having $|j+\rangle$ definitely occupied and $|j-\rangle$ definitely empty; since pair-scattering involving level $j$ is blocked, the energy cost is

$$
\begin{aligned}
& \left.\left(\varepsilon_{j}-\mu\right)-\left[\left(\varepsilon_{j}-\mu\right) 2\left\langle b_{j}^{\dagger} b_{j}\right\rangle\right)-\lambda d \sum_{i(\neq j)}\left\langle b_{i}^{\dagger} b_{j}+b_{j}^{\dagger} b_{i}\right\rangle\right] \\
& =\left(\varepsilon_{j}-\mu\right)\left(1-2 \bar{v}_{j}^{2}\right)+\lambda d \sum_{i(\neq j)}\left(C_{i j}+C_{j i}\right),
\end{aligned}
$$

in which the restriction on the sum reflects the blocking of scattering events involving level $j$. Since the first term of (24) is positive definite (particle-hole symmetry ensures that $\left(\frac{1}{2}-\bar{v}_{j}^{2}\right) \gtrless 0$ if $\varepsilon_{j}-\mu \gtrless 0$ ) and the second of order $\Delta_{\text {can }}$, the excitation energy will be finite even for $d \rightarrow 0$, implying the existence of a gap of order $\Delta_{\text {can }}$. Similarly, "phase-breaking excitations" that violate the fixed-phase condition (ii) are gapped too: for example, if $\left(C_{i j}\right)_{\text {excited }}=-\left(C_{i j}\right)_{\text {ground }}$ for a given $j$ and all $i(\neq j)$, the energy cost is $-\lambda d \sum_{i(\neq j)}\left[\left(C_{i j}+C_{j i}^{*}\right)_{\text {excited }}-\left(C_{i j}+C_{j i}\right)_{\text {ground }}\right]$, which is at least of order $2 \Delta_{\text {can }}$.

We see, therefore, that the essence of pairing correlations can readily be formulated in a canonical framework: (i) a redistribution of occupation probability across $\varepsilon_{\mathrm{F}}$ occurs, such that each level $j$ in a finite range around $\varepsilon_{\mathrm{F}}$ has a finite probability of both being doubly occupied or empty, and (ii) any two components of the ground state wavefunction that differ only by the exchange of a pair of electrons between two levels $i$ and $j$ have the same phase.

Since pairing correlations with these properties are the microscopic property at the heart of all manifestations of "superconductivity", it seems reasonable to call a sample "superconducting" as long as it exhibits pairing correlations with measurable 

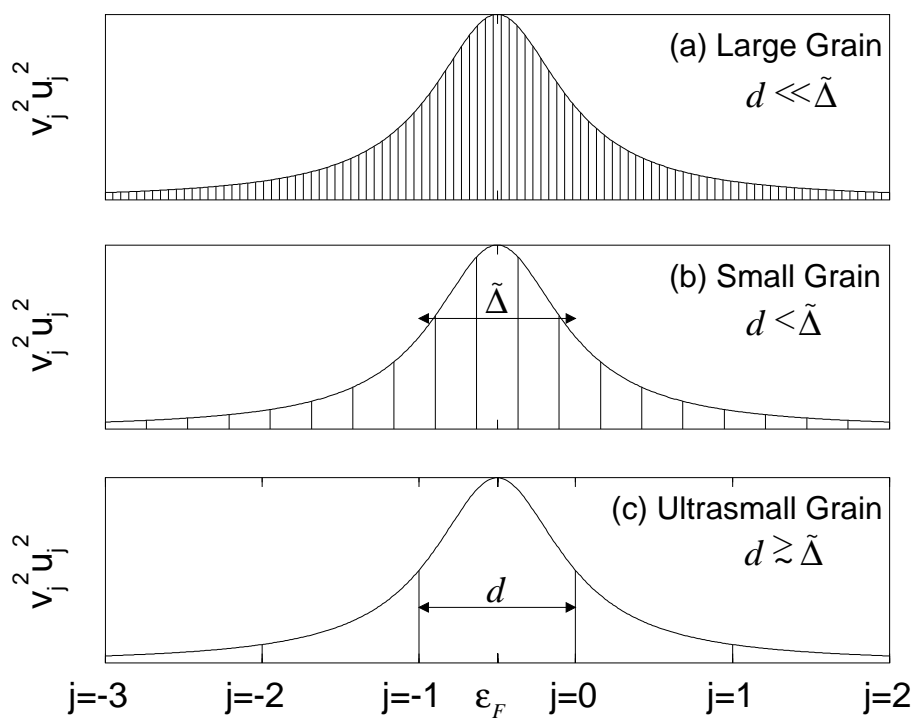

Fig. 7 A cartoon depiction of why "superconductivity breaks down" when the sample becomes sufficiently small. Vertical lines are drawn at each single-particle energy $\varepsilon_{j}$, spaced with a mean level spacing $d$ corresponding to (a) a "large" grain $(d \ll \tilde{\Delta})$; (b) a "small" grain $(d \simeq 0.25 \tilde{\Delta}) ;($ c) an "ultrasmall" grain $(d \simeq \tilde{\Delta})$. The lines' height represents the function $u_{j}^{2} v_{j}^{2}=\frac{1}{4} \tilde{\Delta}^{2} /\left(\varepsilon_{j}^{2}+\tilde{\Delta}^{2}\right)$ of standard bulk BCS theory, to illustrate the energy range (of width $\tilde{\Delta}$ around $\left.\varepsilon_{\mathrm{F}}\right)$ within which pairing correlations are strongest. Loosely speaking, the number of single-particle levels in this regime, namely $\tilde{\Delta} / d$, corresponds to "the number of Cooper pairs" of the system. Evidently, this number becomes less than one when $d \gtrsim \tilde{\Delta}$ as in (c), so that it then no longer makes sense to call the system "superconducting" [cf. Sec. 6.2]

consequences. And by this criterion the gap observed in the even grains of RBT certainly qualifies.

\subsection{Gauge symmetry breaking}

Note that property (ii) will be preserved under the gauge transformation $c_{j \sigma} \rightarrow \mathrm{e}^{\mathrm{i} \phi_{j}^{\prime}} c_{j \sigma}$, i.e. $C_{i j} \rightarrow \mathrm{e}^{-2 \mathrm{i}\left(\phi_{i}^{\prime}-\phi_{j}^{\prime}\right)} C_{i j}$, only if all $\phi_{j}^{\prime}$ are equal, say $\phi_{j}^{\prime}=\phi^{\prime}$. Property (ii), and likewise the pairing parameter $\Delta_{\text {can }}$, therefore (a) are not gauge invariant "locally" in $j$-space, but (b) are gauge invariant globally. These are obvious consequences of the facts that (a) a correlated fixed- $N$ state consists of a phase-coherent superposition of many different components, and hence cannot be invariant under arbitrary changes of the phases of individual components; and that (b) all of these components contain the same number of electrons $N$ and hence under a global gauge transformation all pick up the same phase factor $\mathrm{e}^{\mathrm{i} N \phi^{\prime}}$. Obviously, global gauge symmetry can therefore never be broken in a canonical ensemble. In contrast, the breaking of global gauge symmetry by the g.c. pairing parameter $\Delta_{\text {gc }}$ of Eq. (18), which transforms as $\Delta_{g c} \rightarrow \mathrm{e}^{2 i \phi^{\prime}} \Delta_{\text {gc }}$, is an inevitable consequence or artefact of its g.c. definition [75, p. 142]. 


\subsection{Making contact with standard BCS theory}

One of the breakthrough achievements of BCS was, of course, to propose a simple variational ground state which has precisely the properties (i) and (ii) described above: when evaluating the correlators of Eq. (19) using $|\mathrm{BCS}\rangle$ of Eq. (17), one finds

$$
\left(\bar{u}_{j}\right)_{\mathrm{BCS}}^{2}=u_{j}^{2}, \quad\left(\bar{v}_{j}\right)_{\mathrm{BCS}}^{2}=v_{j}^{2}, \quad\left(C_{i j}\right)_{\mathrm{BCS}}=u_{i} v_{i} u_{j} v_{j} \mathrm{e}^{-\mathrm{i}\left(\phi_{i}-\phi_{j}\right)},
$$

and also $\left(\Delta_{\text {can }}^{2}\right)_{\mathrm{BCS}}=\left|\Delta_{\mathrm{gc}}\right|^{2}$. The definite-phase requirement (ii) can thus be implemented by choosing all the phases $\phi_{j}$ to be the same, say $\phi_{j}=\phi$ for all $j$, thereby breaking local gauge invariance (usually one simply takes $\phi=0$ ); and requirement (i) is fulfilled automatically when minimizing the expectation value $\langle\hat{H}\rangle_{\mathrm{BCS}}$ w.r.t. $u_{j}$ and $v_{j}$, since this does yield smeared-out step functions, namely [1, 58]

$$
v_{j}^{2}=\frac{1}{2}\left[1-\left(\varepsilon_{j}-\mu\right) / E_{j}\right], \quad E_{j} \equiv \sqrt{\left(\varepsilon_{j}-\mu\right)^{2}+\left|\Delta_{\mathrm{gc}}\right|^{2}} .
$$

Here we neglected terms that vanish for $d \rightarrow 0$, and $\Delta_{\mathrm{gc}}$ is determined by the famous gap equation (for $T=0$ ),

$$
\frac{1}{\lambda}=d \sum_{\left|\varepsilon_{j}\right|<\omega_{\mathrm{D}}} \frac{1}{2 E_{j}} .
$$

The BCS wavefunction instructively illustrates some of the general properties discussed above. Firstly, the product $u_{j}^{2} v_{j}^{2}$, shown in Fig. 7, has a bell-shaped form with a welldeveloped peak around $\varepsilon_{\mathrm{F}}$ of width $\simeq\left|\Delta_{\mathrm{gc}}\right|$, illustrating that pairing correlations are strongest within a region of width $\left|\Delta_{\mathrm{gc}}\right|$ around the Fermi surface. Secondly, the energy of a blocking excitation [Eq. (24)] reduces to $\left(\varepsilon_{j}-\mu\right)\left(1-2 v_{j}^{2}\right)+2 u_{j} v_{j}\left|\Delta_{\mathrm{gc}}\right|=E_{j}$, which is just the well-known energy of the Bogoljubov quasiparticle state $\gamma_{j+}^{\dagger}|\mathrm{BCS}\rangle$, where

$$
\gamma_{j \sigma}=u_{j} c_{j \sigma}-\sigma v_{j} e^{\mathrm{i} \phi} c_{j-\sigma}^{\dagger} .
$$

Thirdly, an example of a phase-breaking excitation is

$$
\gamma_{j+}^{\dagger} \gamma_{j-}^{\dagger}|\mathrm{BCS}\rangle=\left(-v_{j} e^{-\mathrm{i} \phi}+u_{j} b_{j}^{\dagger}\right) \prod_{i(\neq j)}\left(u_{i}+v_{j} e^{\mathrm{i} \phi} b_{i}^{\dagger}\right)|\mathrm{Vac}\rangle,
$$

which has $\left(C_{i j}\right)_{\text {excited }}=-u_{i} v_{i} u_{j} v_{j}$ and energy $2 E_{j}$.

It should be appreciated, however, that BCS chose a grand-canonical construction purely for calculational convenience (as is made clear on p. 1180 of their original paper [1]): the trick of using a factorized form of commuting products in (17), at the cost of $N$-indefiniteness, makes it brilliantly easy to determine the variational parameters $u_{j}$ and $v_{j}$. In fact, BCS proposed themselves to use the projection of $|\mathrm{BCS}\rangle$ to fixed $N$ as the actual variational ground state, namely 75

$$
\begin{aligned}
|\mathrm{PBCS}\rangle & \equiv \int_{0}^{2 \pi} \mathrm{d} \phi \mathrm{e}^{-\mathrm{i} \phi N} \prod_{j}\left(u_{j}+\mathrm{e}^{2 \mathrm{i} \phi} v_{j} b_{j}^{\dagger}\right)|\mathrm{Vac}\rangle \\
& =\frac{1}{(N / 2) !}\left(\prod_{j} u_{j}\right)\left(\sum_{j} \frac{v_{j}}{u_{j}} b_{j}^{\dagger}\right)^{N / 2}|\mathrm{Vac}\rangle
\end{aligned}
$$


(PBCS for Projected BCS), which is of the general form of Eq. (11). In the bulk limit $(d / \tilde{\Delta} \ll 1)$, however, it is completely adequate to use $|\mathrm{BCS}\rangle$ : firstly, the relative error which its factorized form causes, by taking the occupation amplitude of level $j$ to be independent of that of level $i$, scales like $1 / N$ 75, pp. 150,163]; and secondly, the fluctuations in its particle number, $\left(\Delta N^{2}\right)_{\mathrm{BCS}} \equiv\left\langle N^{2}\right\rangle_{\mathrm{BCS}}-N^{2}=\sum_{j}\left(2 u_{j} v_{j}\right)^{2}$, are equal to $\pi \tilde{\Delta} / d$ in the bulk limit, in which the relative fluctuations $\left(\Delta N^{2}\right)_{\mathrm{BCS}} / N^{2} \propto$ $d \tilde{\Delta} / \varepsilon_{\mathrm{F}}^{2}$ therefore vanish. Thus, bulk results obtained from $|\mathrm{PBCS}\rangle$ or $|\mathrm{BCS}\rangle$ are essentially identical. In fact, Braun [31, 42 checked by explicit calculation that the functions $\left(\bar{v}_{j}^{2}\right)_{\mathrm{G}},\left(\bar{v}_{j}^{2}\right)_{\mathrm{PBCS}}$ and $v_{j}^{2}$ are practically indistinguishable even for $d / \tilde{\Delta}$ as large as 0.5 [see Sec. 12]. Significant differences do develop between them once $d / \tilde{\Delta}$ increases past 0.5, however, as will be discussed in Sec. 12 .

To end this section, note that Fig. 而 offers a very simple intuitive picture for why pairing correlations weaken with increasing level spacing until, in Anderson's words [41], "superconductivity is no longer is possible" when $d \gtrsim \tilde{\Delta}$ : an increase in level spacing implies a decrease in the number of levels within $\tilde{\Delta}$ of $\varepsilon_{\mathrm{F}}$ for which $u_{j}^{2} v_{j}^{2}$ differs significantly from zero, i.e. a decrease in the number of pairs with significant pairing correlations. This number, namely $\tilde{\Delta} / d$, can roughly speaking be viewed as the "number of Cooper pairs" of the system, and when it becomes less than one, as in Fig. If(c), it no longer makes sense to call the system "superconducting". However, this should not be taken to imply that pairing correlations cease altogether in this regime; remnants of them do persist, in the form of fluctuations, up to arbitrarily large $d / \tilde{\Delta}$, as will be discussed in detail in Sec. 12 .

\section{Generalized variational BCS approach}

In the next several sections we review the generalized variational BCS approach used by Braun et al. 31, 19, 42 to describe the paramagnetic breakdown of superconductivity in nm-scale grains in a magnetic field. This theory produces theoretical excitation spectra that are in good qualitative agreement with the measurements of BRT shown in Fig. 5 and thereby yields the most direct confirmation available of the relevance to experiment of the discrete BCS model. Moreover, it sheds considerable light on how "superconductivity breaks down" (more precisely, how pairing correlations weaken) with increasing $d$ and $h$ : As mentioned in the previous paragraph, in grains with $d \simeq \tilde{\Delta}$ (bulk gap), near the lower size limit [41] of observable superconductivity, the number of free-electron states with strong pairing correlations (those within $\tilde{\Delta}$ of $\varepsilon_{\mathrm{F}}$ ) is of order one. Thus, even in grains in which a spectral gap can still be observed, pairing correlations are expected to become so weak that they might be destroyed by the presence of a single unpaired electron [17. This can be probed directly by turning on a magnetic field, since its Zeeman energy favors paramagnetic states with nonzero total spin.

The theory reviewed below exploits analogies to thin films in a parallel magnetic field 66, 67, but explicitly takes account of the discreteness of the grain's spectrum. Since in RBT's experiments the temperature $T=50 \mathrm{mK}$ is much smaller than all other energy scales $(d, \tilde{\Delta})$, we shall neglect finite-temperature effects and set $T=0$. In Secs. 6.1 the eigenenergies $\mathcal{E}_{\alpha}$ of the grain's lowest-lying eigenstates $|\alpha\rangle$ are calculated 


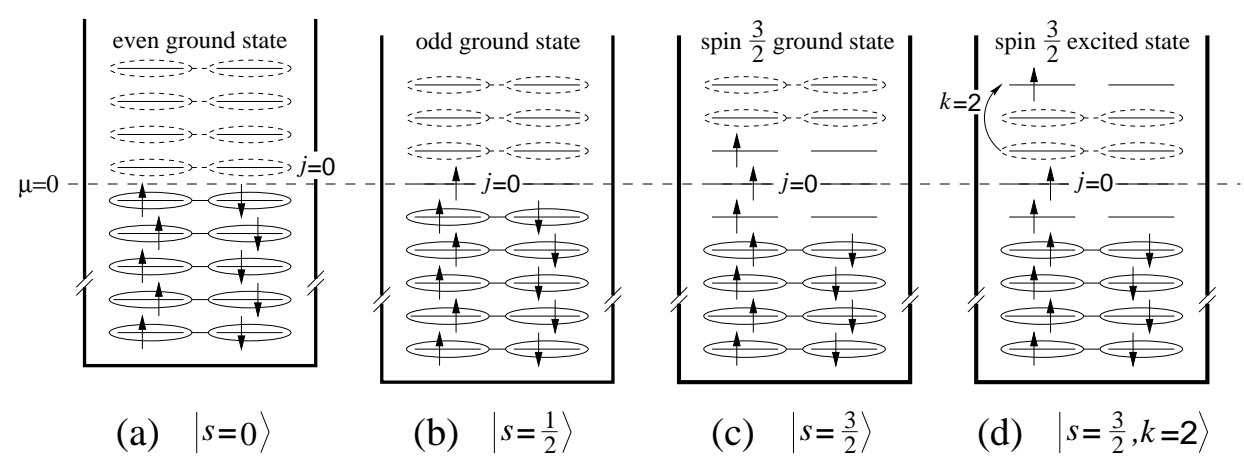

Fig. 8 Cartoon of four typical variational states, labeled using the notation of Eq. (33) for (a-c) and Eq. (34) for (d). They represent (a) the even ground state $|0\rangle$; (b) the odd ground state $\left|\frac{1}{2}\right\rangle ;$ (c) the spin- $\frac{3}{2}$ ground state $\left|\frac{3}{2}\right\rangle ;(\mathrm{d})$ a spin- $\frac{3}{2}$ excited state $\left|\frac{3}{2}, 2\right\rangle$. The single-particle levels are drawn for $h=0$, with the chemical potential half-way between levels 0 and 1 for even systems (a), but exactly on level 0 for odd ones (b,c,d). The ellipses joining states on the same level are meant to represent a "Cooper pair", and signify its being empty or doubly occupied with amplitude $\left(u_{j}+v_{j} b_{j}^{\dagger}\right)$; solid (dashed) ellipses are used for levels that would be completely filled (empty) in the absence of pairing correlations.

approximately using a generalized g.c. variational BCS approach that goes beyond standard mean-field theory by using a different pairing parameter $\Delta_{\alpha}$ for each $|\alpha\rangle$. The $\mathcal{E}_{\alpha}$ are then used to discuss various observable quantities, such as $h$-dependent excitation spectra (Sec. 8) and various parity effects (Sec. 9).

The reasons for deciding to calculate the excitation spectra, despite their fixed $N$ nature, within a grand-canonical framework are as follows: Firstly, its simplicity. Secondly and perhaps most importantly, the exact eigenenergies have the general form $\mathcal{E}_{\alpha}=\mathcal{E}_{n}+\mathcal{E}_{B}(h)$ [Eq. (12)], in which all h-dependence resides in the exactly known contribution $\mathcal{E}_{B}(h)$ from the blocked levels. The choice of approximation scheme therefore only affects $\mathcal{E}_{n}$, which determines the $h=0$ properties of the spectrum, such as the size of the zero-field spectral gap, etc., but not the qualitative features of the $h$-dependence. In particular, this means that all of the analysis below could easily be "made exact" by simply replacing the g.c. approximations for $\mathcal{E}_{n}$ by the exact values from Richardson's solution. However, this is expected to cause only slight quantitative differences, since, thirdly, canonical calculations (mentioned after Eq. (31) and discussed in Sec. 9.4) yield very similar results to g.c. ones as long as $d / \tilde{\Delta} \lesssim 0.5$, which, by inspection of Fig. 5 , does seem to be the case for the grain in question (the analysis of Sec. 8 yields $d / \Delta \simeq 0.67$ ).

\subsection{The generalized variational Ansatz}

The Zeeman term in the Hamiltonian of Eq. (6) favors states with a nonzero total $z$-component of the total spin, $s=\frac{1}{2} \sum_{j \sigma} \sigma c_{j \sigma}^{\dagger} c_{j \sigma}$ (henceforth simply called "spin"). Increasing $h$ will thus eventually lead to a series of ground state changes to states with successively larger spins. In general, therefore, we are interested in pair-correlated 
states with nonzero spin, and in particular in their eigenenergies. Following Braun et al. 18, 19, 42, we now show how this can be calculated variationally, using the following general BCS Ansatz for a state $|s, B\rangle$ with $N=2 n+2 s$ electrons and a definite total spin $s \geq 0$ (first introduced by Soloviev for application in nuclei [74]):

$$
|s, B\rangle=\prod_{i \in B} c_{i+}^{\dagger} \prod_{j}^{U}\left(u_{j}^{(s, B)}+v_{j}^{(s, B)} b_{j}^{\dagger}\right)|\mathrm{Vac}\rangle .
$$

If the spin is nonzero, it is built up by placing $2 s$ unpaired spin-up electrons in a set $B$ of $b=2 s$ single-particle levels [cf. Eq. (10)] while the remaining single-particle levels have BCS-like amplitudes to be either empty $\left(u_{j}^{(s, B)}\right)$ or doubly occupied by a pair $\left(v_{j}^{(s, B)}\right)$, with $\left(u_{j}^{(s, B)}\right)^{2}+\left(v_{j}^{(s, B)}\right)^{2}=1$. The subscript $U$ over products (and over sums below) indicates exclusion of the singly occupied levels in $B$, for which $u^{(s, B)}, v^{(s, B)}$ are not defined. The product $\prod_{j}^{U}$ thus constitutes a g.c. approximation to the state $\left|\Psi_{n}\right\rangle$ of Eq. (11).

More specifically, in a given spin- $s$ sector of Hilbert space the following two types of specializations of Eq. (32) were studied in detail $(p=2 s \bmod 2)$ :

$$
\begin{aligned}
|s\rangle & =\prod_{i=-s+p / 2}^{s-1+p / 2} c_{i+}^{\dagger} \prod_{j}^{U}\left(u_{j}^{s}+v_{j}^{s} b_{j}^{\dagger}\right)|\mathrm{Vac}\rangle . \\
|s, k\rangle & =c_{(s-1+p / 2+k)+}^{\dagger} c_{(s-1+p / 2)+}|s\rangle .
\end{aligned}
$$

$|s\rangle$ is the spin- $s$ state with the lowest energy, i.e. the "variational spin- $s$ ground state", obtained by placing the $2 s$ unpaired electrons as close as possible to $\varepsilon_{\mathrm{F}}$ [Fig. $\left.\mathrm{B}(\mathrm{b}, \mathrm{c})\right]$, in order to minimize the kinetic energy cost of having more spin ups than downs. $|s, k\rangle$ is a particular type of excited spin- $s$ state, obtained from $|s\rangle$ by moving one electron from its topmost occupied level $(s-1+p / 2)$ upwards by $k$ units of $d$ into a higher level $(s-1+p / 2+k)$. These constructions are illustrated in Fig. 8, of which (a) and (b) represent the variational ground states of a grain with an even or odd number of electrons, respectively.

The orthogonality of the wavefunctions, $\left\langle s, B \mid s^{\prime}, B^{\prime}\right\rangle=\delta_{s s^{\prime}} \delta_{B B^{\prime}}$, implies that the variational parameters $v_{j}^{(s, B)}$ and $u_{j}^{(s, B)}$ must be found anew for each $(s, B)$ (hence the superscript), by minimizing the variational "eigenenergies"

$$
\begin{aligned}
\mathcal{E}_{s, B}^{\mathrm{BCS}}(h, d) \equiv\langle s, B|\hat{H}| s, B\rangle & \\
= & -2 s h+\sum_{i \in B}\left(\varepsilon_{i}-\mu\right)+\sum_{j}^{U}\left[2\left(\varepsilon_{j}-\mu\right)\left(v_{j}^{(s, B)}\right)^{2}+\lambda d\left(v_{j}^{(s, B)}\right)^{4}\right] \\
& -\lambda d\left(\sum_{j}^{U} u_{j}^{(s, B)} v_{j}^{(s, B)}\right)^{2}
\end{aligned}
$$

which we use as approximations to the exact eigenenergies $\mathcal{E}_{s, B}^{\text {exact }}(h, d)$. The $v_{j}^{4}$ term is not extensive and hence neglected in the bulk case where only effects proportional to the system volume are of interest. Here it is retained, since in ultrasmall systems it 
is non-negligible (but not dominant either) 18, 19]. Solving the variational conditions $\frac{\partial \mathcal{E}_{s, B}^{\mathrm{BCS}}}{\partial v_{j}^{(s, B)}}=0$ in standard BCS fashion yields

$$
\left(v_{j}^{(s, B)}\right)^{2}=\frac{1}{2}\left(1-\xi_{j} /\left[\xi_{j}^{2}+\Delta_{s, B}^{2}\right]^{1 / 2}\right), \quad \xi_{j} \equiv \varepsilon_{j}-\mu-\lambda d\left(v_{j}^{(s, B)}\right)^{2},
$$

where the "pairing parameter" $\Delta_{s, B}$ is defined by the relation

$$
\Delta_{s, B} \equiv \lambda d \sum_{j}^{U} u_{j}^{(s, B)} v_{j}^{(s, B)}, \quad \text { or } \quad \frac{1}{\lambda}=d \sum_{j}^{U} \frac{1}{2 \sqrt{\xi_{j}^{2}+\Delta_{s, B}^{2}}},
$$

which in the limit $d / \tilde{\Delta} \rightarrow 0$ reduces to the standard bulk $T=0$ gap equation. Note that it is $h$-independent, because it involves only unblocked levels $j \in U$, which are populated by pairs with zero total Zeeman energy. Note also that in Eq. (37) the $\lambda d\left(v_{j}^{(s, B)}\right)^{2}$ shift in $\xi_{j}$, usually neglected because it simply renormalizes the bare energies, is retained, since for large $d$ it somewhat increases the effective level spacing near $\varepsilon_{\mathrm{F}}$ (and its neglect turns out to produce a significant upward shift in the $\mathcal{E}_{s, B}^{\mathrm{BCS}}(h, d)$ 's, which one is trying to minimize).

The chemical potential $\mu$ is fixed by requiring that

$$
2 n+2 s=\langle s, B|\hat{N}| s, B\rangle=2 s+2 \sum_{j}^{U}\left(v_{j}^{(s, B)}\right)^{2} .
$$

In contrast to conventional BCS theory, the pairing parameter $\Delta_{s, B}$ can in general not be interpreted as an energy gap and is not an observable. It should be viewed simply as a mathematical auxiliary quantity which was introduced to conveniently solve the variational conditions. However, by parameterizing $v_{j}^{(s, B)}$ and $u_{j}^{(s, B)}, \Delta_{s, B}$ does serve as a measure of the pairing correlations present in $|s, B\rangle$ : for vanishing $\Delta_{s, B}$ the latter reduces to an uncorrelated paramagnetic state $|s, B\rangle_{0}$ with spin $s$ and energy $\mathcal{E}_{s, B}^{0}$, namely

$$
|s, B\rangle_{0} \equiv \prod_{i \in B} c_{i+}^{\dagger} \prod_{j<0}^{U} b_{j}^{\dagger}|0\rangle, \quad \text { with } \quad \mathcal{E}_{s, B}^{0} \equiv{ }_{0}\langle s, B|\hat{H}| s, B\rangle_{0},
$$

and the condensation energy $E_{s, B}^{\mathrm{cond}} \equiv \mathcal{E}_{s, B}^{\mathrm{BCS}}-\mathcal{E}_{s, B}^{0}$ of $|s, B\rangle$ reduces to zero.

\subsection{General numerical solution - illustration of the blocking effect}

The simultaneous solution of Eqs. (37), (38) and (39) is a straightforward numerical exercise which Braun and von Delft performed 18, 19, for the sake of "numerical consistency", without further approximations. (Analytical solutions can be found only in the limits $d \ll \tilde{\Delta}$ and $d \gg \Delta_{s, B}$, see App. A of [19].) The numerical results are summarized in Fig. 9, which shows the pairing parameters $\Delta_{s, B}$ [Figs. 9(a,c)] and energies $\mathcal{E}_{s, B}^{\mathrm{BCS}}$ [Figs. 9 (b,d), solid lines] of some selected variational states $|s, B\rangle$, as well as the energies $\mathcal{E}_{s, B}^{0}$ of the corresponding uncorrelated states $|s, B\rangle_{0}$ [Figs. 9 (b,d), dashed 

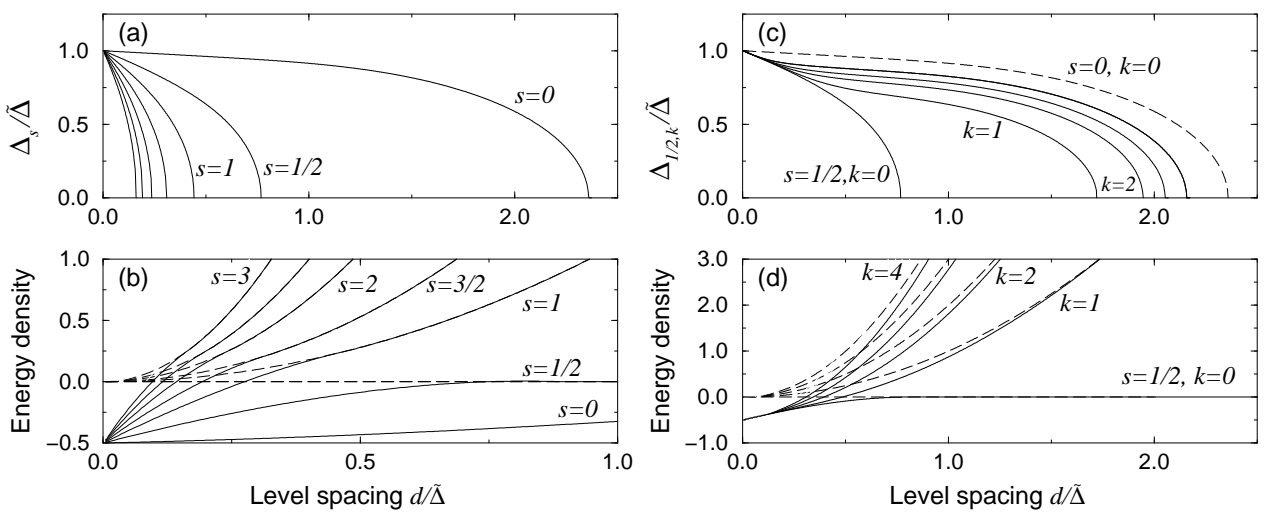

Fig. 9 Properties of (a,b) spin-s ground states $|s\rangle=|s, 0\rangle$ [Eq. (33)] and (c,d) spin- $\frac{1}{2}$ excited states $\left|\frac{1}{2}, k\right\rangle$ (for $k=0, \ldots, 4$ ) [Eq. (34)], as functions of $d / \tilde{\Delta}$ (i.e. decreasing grain size), calculated for $\lambda=0.194$. (a) The pairing parameters $\Delta_{s}(d) / \tilde{\Delta}$, which vanish at critical level spacings $d_{s}^{\mathrm{BCS}}$ of $2.36,0.77,0.44,0.31, \ldots$ for $s=0,1 / 2,1,3 / 2, \ldots$, respectively. (c) The pairing parameters $\Delta_{1 / 2, k}$, together with their $k \rightarrow \infty$ limit, which equals $\Delta_{0}$ (dashed line). (b,d) show relative energy densities (since normalized by $d / \tilde{\Delta}^{2} \propto \mathrm{Vol}^{-1}$ ) at $h=0$ for both correlated (solid) and uncorrelated (dashed) states, the latter obtained by setting $\Delta_{s, B}=0$ in the former. (b) shows $\left(\mathcal{E}_{s}^{\mathrm{BCS}}-\mathcal{E}_{p / 2}^{0}\right) d / \tilde{\Delta}^{2}$ (solid) and $\left(\mathcal{E}_{s}^{0}-\mathcal{E}_{p / 2}^{0}\right) d / \tilde{\Delta}^{2}$ (dashed), the energy differences of $|s\rangle$ and $|s\rangle_{0}$ relative to the uncorrelated spin- $p / 2$ Fermi sea $|p / 2\rangle_{0}$. (d) shows $\left(\mathcal{E}_{1 / 2, k}^{\mathrm{BCS}}-\mathcal{E}_{1 / 2}^{0}\right) d / \tilde{\Delta}^{2}$ (solid) and $\left(\mathcal{E}_{1 / 2, k}^{0}-\mathcal{E}_{1 / 2}^{0}\right) d / \tilde{\Delta}^{2}$ (dashed), the energy differences of $\left|\frac{1}{2}, k\right\rangle$ and $\left|\frac{1}{2}, k\right\rangle_{0}$ relative to the uncorrelated spin- $\frac{1}{2}$ ground state $\left|\frac{1}{2}, 0\right\rangle_{0}=\left|\frac{1}{2}\right\rangle_{0}$. Solid and dashed lines meet at the critical level spacing $d_{s, k}^{\mathrm{BCS}}$ at which $\Delta_{s, k}$ becomes 0 and the condensation energy $E_{s, k}^{\mathrm{cond}}=\mathcal{E}_{s, k}^{\mathrm{BCS}}-\mathcal{E}_{s, k}^{0}$ vanishes.

lines]; both $\mathcal{E}_{s, B}^{\mathrm{BCS}}$ and $\mathcal{E}_{s, B}^{0}$ are plotted relative to the energy $\mathcal{E}_{p / 2}^{0}$ of the uncorrelated spin- $p$ Fermi sea $|p / 2\rangle$. The results have a number of salient features:

(i) In the bulk limit $d / \tilde{\Delta} \rightarrow 0$, all of the pairing parameters $\Delta_{s, B}$ reduce to $\tilde{\Delta}$, as expected, and the energy differences $\mathcal{E}_{s, B}^{\mathrm{BCS}}-\mathcal{E}_{p / 2}^{0}$ between the correlated states $|s, B\rangle$ and the uncorrelated Fermi sea $|p / 2\rangle$ reduce to $-\frac{1}{2} \tilde{\Delta}^{2} / d=-\frac{1}{2} \mathcal{N}\left(\varepsilon_{\mathrm{F}}\right) \tilde{\Delta}^{2}$, which is the standard bulk result for the condensation energy.

(ii) Each $\Delta_{s, B}$ in Figs. 9 (a,c) decreases with increasing $d$. This reflects the fact that with increasing $d$, the number of pair-correlated states within $\tilde{\Delta}$ of $\varepsilon_{\mathrm{F}}$ decreases [cf. Fig. 7 and the last paragraph of Sec. 5.5, so that the amount of pairing correlations, for which $\Delta_{s, B}$ is a measure, decreases too.

(iii) Each $\Delta_{s, B}$ vanishes abruptly at a critical level spacing $d_{s, B}^{\mathrm{BCS}}$ (whose precise numerical value depends sensitively on model assumptions such as the value of $\lambda$ and the use of uniformly-spaced levels [20]). For $d>d_{s, B}^{\mathrm{BCS}}$ no pairing correlations exist at this level of approximation, so that that the condensation energy $E_{s, B}^{\text {cond }}$ (difference between solid and dashed lines) vanishes and the solid and dashed lines in Figs. 9(b,d) meet.

(iv) In Figs. 9(a), the pairing parameters $\Delta_{s}$ for the spin- $s$ ground states decrease 
rapidly with increasing $s$ at fixed $d$ (and $d_{s}^{\mathrm{BCS}}<d_{s^{\prime}}^{\mathrm{BCS}}$ if $s>s^{\prime}$ ). [This is a generalization of a parity effect discussed by von Delft et al. [17], who studied only ground state pairing correlations and found that these are weaker in odd $(s=1 / 2)$ grains than in even $(s=0)$ grains, $\Delta_{1 / 2}<\Delta_{0}$, cf. Sec. 9.] This tendency is a direct consequence of the blocking effect described in Sec. 1.4 and is independent of model details: larger $s$ means more unpaired electrons, more terms missing from the sum $\sum_{j}^{U}$, less correlated pairs and hence smaller $\Delta_{s, B}$.

(v) As $d$ increases the blocking effect described in (iv) becomes stronger, i.e. the difference between the various $\Delta_{s}$ for different $s$ becomes more pronounced, since then the relative weight of each term missing in the sum $\sum_{j}^{U}$ increases. The blocking effect is most dramatic in the regime $d / \tilde{\Delta} \in[0.77,2.36]$ in which $\Delta_{0} \neq 0$ but $\Delta_{s \neq 0}=0$. This is a regime of "minimal superconductivity" 18, 19], in the sense that all pairing correlations that still exist in the even variational ground state $|0\rangle$ (since $\Delta_{0} \neq 0$ ) are completely destroyed by the addition of a single electron or the flipping of a single spin ( since $\Delta_{s \neq 0}=0$ ).

(vi) Considering the spin- $\frac{1}{2}$ excited states $\left|\frac{1}{2}, k\right\rangle$ of Figs. 9(b,d), one finds that the larger $k$, the longer the pairing correlations survive with increasing $d$ : the critical spacings $d_{1 / 2, k}^{\mathrm{BCS}}$ increase with $k$, approaching the value $d_{0}^{\mathrm{BCS}}$ of the spin-0 case as $k \rightarrow \infty$; correspondingly, the larger $k$, the larger the $d$-value at which the condensation energies $E_{1 / 2, k}^{\text {cond }}$ [differences between solid and dashed lines in Fig. 9(d)] vanish. The intuitive reason why the amount of pairing correlations in an excited $|s, k\rangle$ increases with $k$ is of course quite simple: the further the unpaired electron sits from the Fermi surface where pairing correlations are strongest, the less it disrupts the latter (since $u_{k} v_{k}$ becomes very small for large $k$, see Fig. 7). In fact, the state $\left|\frac{1}{2}, k \rightarrow \infty\right\rangle$ will have just about the same amount of pairing correlations as the even ground state $|0\rangle$ $\left(\Delta_{1 / 2, k \rightarrow \infty} \simeq \Delta_{0}\right)$.

(vii) Similar effects hold for excited states in other spin sectors (not shown): The higher the excitation, the larger the pairing parameter $\Delta_{s, B}$. However, the concomittant gain in correlation energy is always less than the kinetic-energy cost of having an unpaired electron far from $\varepsilon_{\mathrm{F}}$.

(viii) The strong dependence of $\Delta_{s, B}$ on $s$ and $d$ for $d \gtrsim \tilde{\Delta}$ illustrates why in this regime a conventional mean-field treatment is no longer sufficient: the system cannot be characterized by a single pairing parameter, since the amount of pairing correlations vary from state to state, each of which is characterized by its own pairing parameter. Instead, the present variational approach is, roughly speaking, equivalent to a doing a separate mean-field calculation for each new choice $U$ of unblocked levels within the Fock space spanned by them (i.e. replacing $b_{j} \rightarrow\left\{b_{j}-\left\langle b_{j}\right\rangle\right\}+b_{j}$ and neglecting terms quadratic in the fluctuations $\left.\left\{b_{j}-\left\langle b_{j}\right\rangle\right\}\right)$. Indeed, the behavior of $\Delta_{s, B}(d)$ near $d_{s, B}^{\mathrm{BCS}}$ has the standard mean-field form $\sqrt{1-d / d_{s, B}^{\mathrm{BCS}}}$, as can be shown analytically [19, App. A].

To summarize: pairing correlations decrease with increasing $d$ and $s$ and decreasing $k$. These features survive also in more accurate canonical calculations. This is not the case, however, for the abrupt vanishing of $\Delta_{s, B}$ at $d_{s, B}^{\mathrm{BCS}}$, which signals the breakdown of the g.c. approach once $d$ becomes of order $\tilde{\Delta}$ : canonical methods show that, regardless how large $d$ becomes, some remnants of pairing correlations survive and the pairing 
parameters $\left(\Delta_{s, B}\right)_{\text {can }}$ do not vanish [Sec. 9.4], in accordance with the rule of thumb that "in a finite system no abrupt phase transition can occur between a zero and nonzero order parameter."

\section{Softening of the $H$-induced transition to a paramagnetic state}

Since states with nonzero spin are favored by the Zeeman energy but have smaller correlation energy due to the blocking effect, a competition arises between Zeeman energy and correlation energy. The manifestations of the blocking effect can thus be probed by turning on a magnetic field; if it becomes large enough to enforce a large spin, excessive blocking will destroy all pairing correlations.

The situation is analogous to ultra-thin films in a parallel magnetic field 66, 67, where orbital diamagnetism is negligible for geometrical reasons and superconductivity is destroyed at sufficiently large $h$ by Pauli paramagnetism. This occurs via a first order transition to a paramagnetic state, as predicted by Clogston and Chandrasekhar (CC) [80, 81 by the following argument (for bulk systems): A pure Pauli paramagnet chooses its spin $s$ such that the sum of the kinetic and Zeeman energies, $s^{2} / \mathcal{N}\left(\varepsilon_{\mathrm{F}}\right)-2 h s$, is minimized, and hence has spin $s=h \mathcal{N}\left(\varepsilon_{\mathrm{F}}\right)$ and ground state energy $-h^{2} \mathcal{N}\left(\varepsilon_{\mathrm{F}}\right)$. When this energy drops below the bulk correlation energy $-\frac{1}{2} \tilde{\Delta}^{2} \mathcal{N}\left(\varepsilon_{\mathrm{F}}\right)$ of the superconducting ground state, which happens at the critical field $h_{\mathrm{CC}}=\tilde{\Delta} / \sqrt{2}$, a transition will occur from the superconducting to the paramagnetic ground state. The transition is first-order, since the change in spin, from 0 to $s_{\mathrm{CC}}=h_{\mathrm{CC}} \mathcal{N}\left(\varepsilon_{\mathrm{F}}\right)=\tilde{\Delta} /(d \sqrt{2})$, is macroscopically large $\left(\mathcal{N}\left(\varepsilon_{\mathrm{F}}\right)=1 / d \propto \mathrm{Vol}\right)$.

This transition has been directly observed by Meservey and Tedrow [66, 67] in ultra-thin $(5 \mathrm{~nm})$ superconducting Al films $(\tilde{\Delta}=0.38 \mathrm{meV})$, whose density of states [Fig. 10(a)] they measured via the tunnel conductance through an oxide layer between a normal metal and the film. They found that in a magnetic field the BCS quasiparticle peak splits up into two subpeaks, separated in energy by $2 \mu_{\mathrm{B}} H$ [Fig. 10(b)], which simply reflects the Zeeman splitting of quasiparticles states $\mathrm{f}^{\text {[ }}$ with spin up or down (and $g=2$ ). Remarkably, the tunneling threshold abruptly dropped to zero at a field of $4.7 \mathrm{~T}$ [Fig. 10(b)], which they associated with the field $H_{\mathrm{CC}}$ at which the phase transition from the superconducting to the paramagnetic ground state occurs. Indeed, Fig. 10(b) demonstrates clearly that the transition to the normal state is first order: the mean of the spin-up and spin-down spectral gaps, i.e. the pairing parameter $\tilde{\Delta}$, is constant until the critical field $H_{\mathrm{CC}}$ is reached, at which it abruptly drops to zero.

For the case of isolated ultrasmall grains, the above picture of the transition needs to be rethought in two respects due to the discreteness of the electronic spectrum: Firstly, the spin must be treated as a discrete (instead of continuous) variable, whose changes with increasing $h$ can only take on (parity-conserving) integer values. Secondly, one needs to consider more carefully the possibility of $h$-induced transitions to nonzero spin states that are still pair-correlated (instead of being purely paramagnetic), such as the variational states $|s, B\rangle$ discussed above. (In the bulk case, it is

\footnotetext{
${ }^{\mathrm{h}}$ Recall that the BCS quasiparticles $\gamma_{j, \sigma}^{\dagger}=u_{j} c_{j, \sigma}^{\dagger}-\sigma v_{j} c_{j,-\sigma}$ have well-defined spins.
} 

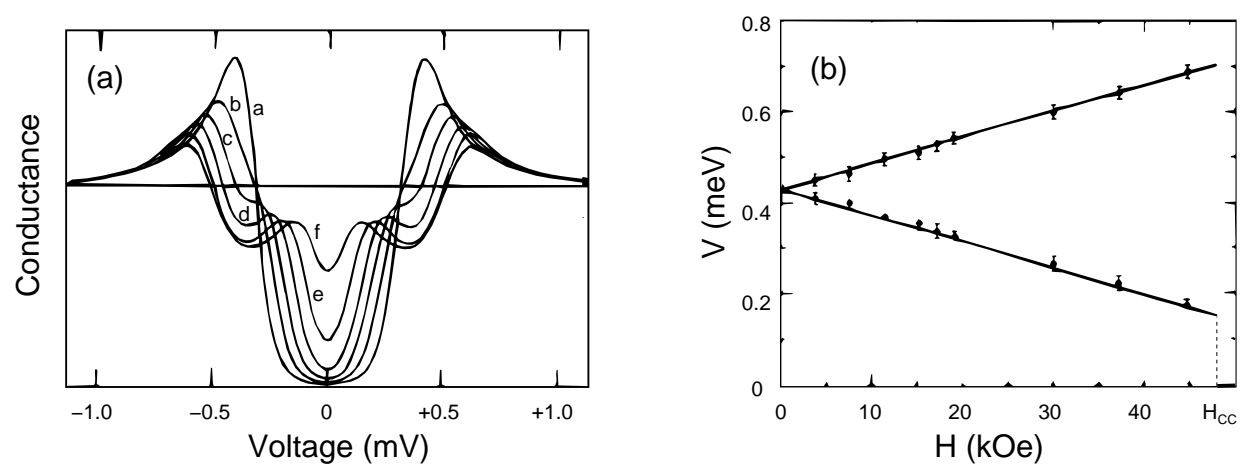

Fig. 10 Thin films in a magnetic field (Figs. 11 and 12 of 66 ). (a) Tunneling conductance from a normal metal through a tunnel barrier into a thin superconducting Al film, as function of voltage, for several magnetic fields labeled in increasing order "a" to "f". The conductance reflects the BCS quasiparticle density of states, whose single peak (for a given sign of $V$ ) for $H=0$ splits into two separate peaks for $H \neq 0$, corresponding to the Zeeman energy difference between quasiparticles with spin up and down. (b) Voltage corresponding to the maxima of spin-up and spin-down density of states as a function of magnetic field. At the critical field $H_{\mathrm{CC}}$ superconductivity is destroyed and the tunneling threshold drops abruptly to zero.

obvious that such states play no role: the lowest pair-correlated state with nonzero spin obtainable from the ground state by spin flips is a two-quasiparticle state, costing energy $2 \tilde{\Delta}-2 h$; when $h$ is increased from 0 , the paramagnetic transition at $h_{\mathrm{CC}}=\tilde{\Delta} / \sqrt{2}$ thus occurs before a transition to this state, which would require $h=\tilde{\Delta}$, can occur.)

Quite generally, the effect of increasing $h$ from 0 can be analyzed as follows: At given $d$ and $h$, the grain's ground state is the lowest-energy state among all possible spin- $s$ ground states $|s\rangle$ having the correct parity $p=2 s \bmod 2$. Since $\mathcal{E}_{s}(h, d)=$ $\mathcal{E}_{s}(0, d)-2 h s$, level crossings occur with increasing $h$, with $\mathcal{E}_{s^{\prime}}$ dropping below $\mathcal{E}_{s}$ at the level crossing field

$$
h_{s, s^{\prime}}(d)=\frac{\mathcal{E}_{s^{\prime}}(0, d)-\mathcal{E}_{s}(0, d)}{2\left(s^{\prime}-s\right)} .
$$

Therefore, as $h$ is slowly turned on from zero with initial ground state $\left|s_{0}=p / 2\right\rangle$, a cascade of successive ground-state changes (GSCs) to new ground states $\left|s_{1}\right\rangle,\left|s_{2}\right\rangle$, . . will occur at the fields $h_{s_{0}, s_{1}}, h_{s_{1}, s_{2}}, \ldots$ Let us denote this cascade by $\left(s_{0}, s_{1}\right) ;\left(s_{1}, s_{2}\right) ; \ldots$; for each of its GSCs the corresponding level-crossing fields $h_{s, s^{\prime}}(d)$ is shown in Fig. 11 . Generalizing CC's critical field to nonzero $d$, let us denote the (parity-dependent) field at which the first transition $\left(s_{0}, s_{1}\right)$ occurs by $h_{\mathrm{CC}}(d, p) \equiv h_{s_{0}, s_{1}}(d)$, which simply is the lower envelope of the level-crossing fields $h_{s_{0}, s_{1}}$ in Fig. 11 (shown as bold solid and dashed lines for $s_{0}=0$ and $s_{0}=\frac{1}{2}$, respectively). In the limit $d \rightarrow 0$ it is numerically found to reduce to the Clogston-Chandrasekhar value, i.e. $h_{\mathrm{CC}}(0, p)=\tilde{\Delta} / \sqrt{2}$, as expected.

In general, the order in which the GSCs occur with increasing $h$ within a cascade (i.e. the order of $h_{s, s^{\prime}}$ lines encountered when moving vertically upward in Fig. 11) 


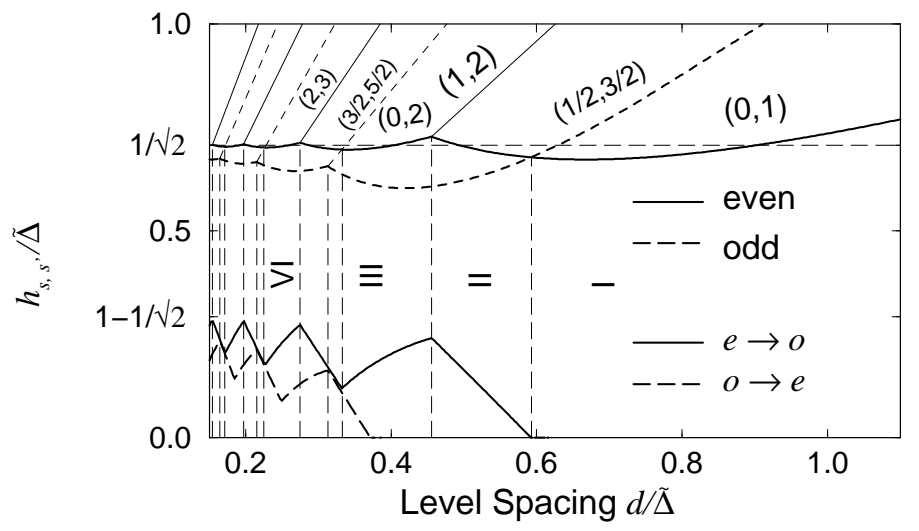

Fig. $11 d$-dependence of the level-crossing fields $h_{s, s^{\prime}}(d) / \tilde{\Delta}$ [Eq. (41)] at which $\mathcal{E}_{s^{\prime}}^{\mathrm{BCS}}$ drops below $\mathcal{E}_{s}^{\mathrm{BCS}}$ with increasing $h$. Only those level crossing fields are shown that belong to the cascade of ( fixed- $N)$ ground state changes (GSCs) $\left(s_{0}, s_{1}\right) ;\left(s_{1}, s_{2}\right) ; \ldots$ that occur as $h$ increases from 0 at given $d$. Solid (dashed) lines are used for even (odd) grains with integer (half-integer) spins, and some are labeled by the associated GSC $\left(s, s^{\prime}\right)$. (In contrast, in Fig. 12 the $N$-changing tunneling transitions in are labeled by $\left|s_{i}\right\rangle \rightarrow\left|s_{f}\right\rangle$.) The size $\left|\Delta \mathcal{E}_{s_{1}, f^{\prime}}-\Delta \mathcal{E}_{s_{0}, f}\right|$ of the first jump (occuring at the level-crossing field $h_{\mathrm{CC}}(p, d)=h_{s_{0}, s_{1}}$ ) in the lowest line of the tunneling spectra of Fig. 12 is shown by the lowest two (jagged) curves (solid for $e \rightarrow o$ and dashed for $o \rightarrow e$ tunneling spectra), which both approach the CC value $1-1 / \sqrt{2}$ as $d \rightarrow 0$.

depends sensitively on $d$ and an infinite number of distinct regimes (cascades) I, II, III, ... can be distinguished: Starting at large $d$ we find the typical normal-grain behavior $(0,1) ;(1,2) ;(2,3) ; \ldots$ for even grains and $\left(\frac{1}{2}, \frac{3}{2}\right) ;\left(\frac{3}{2}, \frac{5}{2}\right) ; \ldots$ for odd grains, with $h_{0,1}<$ (or $>$ ) $h_{\frac{1}{2}, \frac{3}{2}}$ in regimes I (or II). In regimes III and IV of somewhat smaller $d$, the order of GSCs is $(0,2) ;(2,3) ; \ldots$ and $\left(\frac{1}{2}, \frac{3}{2}\right) ;\left(\frac{3}{2}, \frac{5}{2}\right) ; \ldots$, etc, i.e. the spin $s_{1}$ attained after the first GSC $\left(s_{0}, s_{1}\right)$ has increased to 2 in the even case. This illustrates a general trend: the spin $s_{1}(d)$ after the first transition increases with decreasing $d$ and becomes macroscopically large in the $d \rightarrow 0$ limit, where $s_{1}=h_{\mathrm{CC}} / d=\tilde{\Delta} /(d \sqrt{2})$, as explained in recounting CC's argument above.

Furthermore, it turns out that $\Delta_{s_{1}}(d)=0$ and therefore $\mathcal{E}_{s_{1}}^{\mathrm{BCS}}=0$ for all $d$, implying that after the first GSC the new ground state $\left|s_{1}\right\rangle$ is always (not only in CC's bulk limit) a purely paramagnetic state, i.e. without any pairing correlations in the g.c. framework (canonical calculations would yield some weak remnant pairing correlations in the form of fluctuations). In this regard, CC's picture of the transition remains valid throughout as $d$ is increased: at $h_{\mathrm{CC}}(d, p)$, a transition occurs from the superconducting ground state to a paramagnetic, uncorrelated state $\left|s_{1}\right\rangle_{0}$, the transition being first-order in the sense that $\Delta_{s_{1}}(d)=0$; however, the first-order transition is "softened" with increasing d, in the sense that the size of the spin change, $s_{1}-s_{0}$, decreases from being macroscopically large in the bulk to being equal 1 at $d \gg \tilde{\Delta}$ (regimes I and II).

To conclude this section, we mention that the above analysis of the paramagnetic 
breakdown of superconductivity has recently been generalized to finite temperatures [28], using the so-called static path approximation [explained in Sec. 14.3] to treat fluctuation effects properly.

\section{Excitation spectrum in a magnetic field}

In this section we compare the theoretical tunneling spectra for a grain coupled to leads, calculated as functions of $h$ and $d$ [18, 19, 42], and compare these to RBT's measurements of Fig. 5 .

The form of the tunneling spectrum depends in a distinct way on the specific choice of level spacing $d$ and on the electron number parity $p$ of the final states $|f\rangle$ of the bottleneck tunneling processes $|i\rangle \rightarrow|f\rangle$ (or $\left|\alpha^{\prime}\right\rangle \rightarrow|\alpha\rangle$ in the notation of Sec. 2). However, for the uniformly spaced $\varepsilon_{j}$-levels used here, particle-hole symmetry ensures that there is no difference between electron addition or removal spectra $\left|i_{N \mp 1}\right\rangle \rightarrow\left|f_{N}\right\rangle$. To calculate the spectrum for given $d$ and $p$, Braun et al. 18, 19, 42] proceeded as follows: they first analyzed at each magnetic field $h$ which tunneling processes $|i\rangle \rightarrow|f\rangle$ are possible, then calculated the corresponding tunneling energy thresholds $\Delta \mathcal{E}_{i f}(h) \equiv \mathcal{E}_{f}(h)-\mathcal{E}_{i}(h)$ [cf. Eq. (田)] and plotted $\Delta \mathcal{E}_{i f}(h)-\Delta \mathcal{E}_{\min }(0)$ as functions of $h$ for various combinations of $|i\rangle$ and $|f\rangle$, each of which gives a line in the spectrum. Since the selection rule $s_{f}-s_{i}= \pm 1 / 2$ holds, only slopes of \pm 1 can occur. The reason for subtracting $\Delta \mathcal{E}_{\text {min }}(0)$, the $h=0$ threshold energy cost for the first (lowestlying) transition, is that in experiment, this energy depends on $V_{g}$ and hence yields no significant information (see also Sec. 2.4.6 of Ref. [16]). Neglecting nonequilibrium effects [7, 8, 9, 10] (which were minimized in the present experiment by tuning $V_{\mathrm{g}}$ ), the initial state is always taken to be the ground state of a given spin- $s$ sector. The appropriate $s_{i}(h, d)$ must be determined from Fig. 11 .

Fig. 12 shows four typical examples of such theoretical tunneling spectra, with some lines labeled by the corresponding $|i\rangle \rightarrow|f\rangle$ transitions. Whenever $h$ passes through one of the level-crossing fields $h_{s_{i}, s_{i^{\prime}}}$ of Eq. (41), the grain experiences a ground state change $\left(s_{i}, s_{i^{\prime}}\right)$, at which the set of allowed tunneling transitions changes from $\left|s_{i}\right\rangle \rightarrow\left\{\left|s_{f}\right\rangle\right\}$ to $\left|s_{i^{\prime}}\right\rangle \rightarrow\left\{\left|s_{f^{\prime}}\right\rangle\right\}$. Therefore, at $h_{s_{i}, s_{i^{\prime}}}$ one set of lines in the tunneling spectrum ends and another begins, producing kinks or discontinuities. A kink occurs if one of the new final states coincides with one of the old ones, $\left|f^{\prime}\right\rangle=|f\rangle$, meaning that it can be reached from both $\left|s_{i}\right\rangle$ and $\left|s_{i^{\prime}}\right\rangle$ [i.e. $s_{f}-s_{i}=-\left(s_{f}-s_{i^{\prime}}\right)$ ], in which case $\Delta \mathcal{E}_{i f}(h)$ and $\Delta \mathcal{E}_{i f^{\prime}}(h)$ have slopes of opposite sign. However, for most lines this is not the case, so that at $h_{s_{i}, s_{i}^{\prime}}$ the line $\left|s_{i}\right\rangle \rightarrow|f\rangle$ simply ends while new lines $\left|s_{i^{\prime}}\right\rangle \rightarrow\left|f^{\prime}\right\rangle$ begin. This results in discontinuities (or "jumps") in the spectrum at $h_{s_{i}, s_{i}^{\prime}}$ of size $\left(\Delta \mathcal{E}_{i^{\prime} f^{\prime}}-\Delta \mathcal{E}_{i f}\right)\left(h_{s_{i}, s_{i}^{\prime}}\right)$, unless by chance some other final state $\left|f^{\prime}\right\rangle$ happens to exist for which this difference equals zero.

Since the order in which the GSCs $\left(s_{i}, s_{i^{\prime}}\right)$ occur as functions of increasing $h$ depend on $d$ and $p$, as indicated by the distinct regimes I, II, III, ... in Fig. 11, one finds a distinct kind of tunneling spectrum for each regime, differing from the others in the positions of its jumps and kinks. In regime I, where the order of occurrence of GSCs with increasing $h$ is $(0,1) ;\left(\frac{1}{2}, \frac{3}{2}\right) ;(1,2) ;\left(\frac{3}{2}, \frac{5}{2}\right) ; \ldots$, there are no discontinuities in the evolution of the lowest line [see Fig. 12(a)]. For example, for the $e \rightarrow o$ spectrum, the 

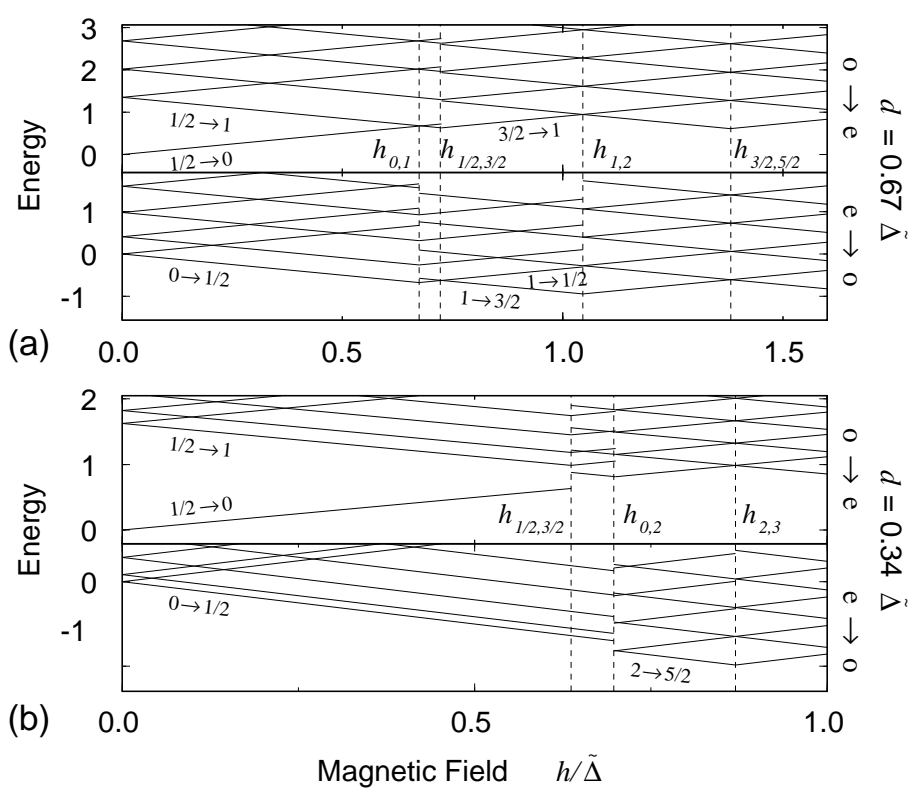

Fig. 12 The theoretical odd-to-even and even-to-odd tunneling spectra $\left(\Delta \mathcal{E}_{i f}-\Delta \mathcal{E}_{\min }(0)\right) / \tilde{\Delta}$ predicted for an ultrasmall superconducting grain as a function of magnetic field $h$, to be compared with the experimental spectra of Fig. 5, for two different level spacings: (a) $d=$ $0.67 \tilde{\Delta}$ and (b) $d=0.34 \tilde{\Delta}$ (corresponding to regimes I and III of Fig. 11, respectively). Some lines are labeled by the corresponding $s_{i} \rightarrow s_{i}^{\prime}$ tunneling transitions. Not all possible higher lines (corresponding to excited final states $|s, j\rangle$ ) are shown. Vertical dashed lines indicate those level-crossing fields $h_{s, s^{\prime}}$ [Eq. (41)] at which kinks or jumps occur, with $h_{0,1}<h_{1 / 2,3 / 2}<$ $h_{1,2}<h_{3 / 2,5 / 2}$ in (a) and $h_{1 / 2,3 / 2}<h_{0,2}<h_{2,3}$ in (b).

lowest $|0\rangle \rightarrow|1 / 2\rangle$ line changes continuously to $|1\rangle \rightarrow|1 / 2\rangle$ at $h_{0,1}$, since $\left|s_{f}-s_{i}^{\prime}\right|=1 / 2$. However, in all other regimes the first change in ground state spin (at $h_{0, s_{1}}$ from 0 to $s_{1}$ ) is $>1$, implying a jump (though possibly small) in all $e \rightarrow o$ lines, as illustrated by Fig. 12 (b).

The jump's magnitude for the tunneling thresholds, i.e. the lowest $e \rightarrow o$ and $o \rightarrow e$ lines, is shown as function of $d$ in the lower part of Fig. 11. It starts at $d=0$ from the CC value $\tilde{\Delta}(1-1 / \sqrt{2})$ measured for thin $\mathrm{Al}$ films [66, 67], and with increasing $d$ decreases to 0 (non-monotonically, due to the discrete spectrum). This decrease of the size of the jump in the tunneling threshold reflects the fact, discussed in Sec. 7, that the change in spin at the first ground state change $\left(s_{0}, s_{1}\right)$ decreases with increasing $d$ (as $s_{1}-s_{0} \sim h_{\mathrm{CC}} / d$ ), and signals the softening of the first-order superconducting-toparamagnetic transition.

The fact that the measured tunneling thresholds in Fig. 5 show no jumps at all, which might at first seem surprising when contrasted to the threshold jumps seen at $h_{\mathrm{CC}}$ in Fig. 10 for thin films in a parallel field [66, 67, can therefore naturally be explained 18, 19 by assuming the grain to lie in the "minimal superconductivity" regime I of Fig. 11 (where the jump size predicted in Fig. 11 is zero). Indeed, the 
overall evolution (i.e. order and position of kinks, etc.) of the lowest lines of Fig. 5 qualitatively agrees with those of a regime I tunneling spectrum, Fig. 19(a). This important result rather convincingly establishes the phenomenological success of the discrete BCS model. It also allows one to deduce the following values for the levelcrossing fields $H_{s_{i}, s_{i}^{\prime}}$ (indicated by vertical dashed lines in Figs. 5 and 12): $H_{0,1}=4 \mathrm{~T}$, $H_{1 / 2,3 / 2}=4.25 \mathrm{~T}, H_{1,2}=5.25 \mathrm{~T}$ and $H_{3 / 2,5 / 2}=6.5 \mathrm{~T}$. As corresponding uncertainties we take $\Delta H_{s_{i}, s_{i}^{\prime}}=0.13 \mathrm{~T}$, which is half the $H$ resolution of $0.25 \mathrm{~T}$ used in experiment.

By combining the above $H_{s_{i}, s_{i}^{\prime}}$ values with Fig. 11, some of the grain's less-wellknown parameters can be determined somewhat more precisely:

(i) To estimate the grain's "bulk $H_{\mathrm{CC}}$ ", note that since $H_{1 / 2,3 / 2} / H_{0,1} \simeq 1.06$, this grain lies just to the right of the boundary between regions II and $\mathrm{I}$ in Fig. 11 where $d / \tilde{\Delta} \simeq 0.63$, at which we have $h_{0,1} / h_{\mathrm{CC}} \simeq 0.95$, so that $H_{\mathrm{CC}}=$ $H_{0,1} / 0.95 \simeq 4.2 \mathrm{~T}$. This is quite close to the value $H_{\mathrm{CC}} \simeq 4.7 \mathrm{~T}$ found experimentally 66, 67 in thin films in a parallel field, confirming our expectation that these correspond to the "bulk limit" of ultrasmall grains as far as paramagnetism is concerned.

(ii) The grain's corresponding bulk gap is $\tilde{\Delta}=\sqrt{2} \mu_{B} H_{\mathrm{CC}} \simeq 0.34 \mathrm{meV}$, implying a coupling constant of $\lambda=0.189$ [by Eq. (8)]. A posteriori, these values can be regarded as being more appropriate for the present grain than the choices $\tilde{\Delta}=0.38 \mathrm{meV}$ and $\lambda=0.194$ made in Sec. 6.2, though the differences are evidently not significant ( $12 \%$ for $\tilde{\Delta}$ and $3 \%$ for $\lambda$ ).

(iii) The mean level spacing implied by $d / \tilde{\Delta} \simeq 0.63$ is $d \simeq 0.21 \mathrm{meV}$. The crude volume-based value $d \simeq 0.45 \mathrm{meV}$ cited in the caption of Fig. 2 thus seems to have been an overestimate. It would be useful if this determination of $d$ could be checked via an independent accurate experimental determination of $d$ directly from the spacing of lines in the excitation spectrum. Regrettably, this is not possible: the measured levels are shifted together by pairing interactions, implying that their spacing does not reflect the mean independent-electron level spacing $d$. Nevertheless, note that the measured spacing of $0.05 \mathrm{meV}$ between the lowest two states of the odd grain agrees quite well with the crude BCS estimate $\sqrt{\tilde{\Delta}^{2}+d^{2}}-\tilde{\Delta}$ [cf. Eq. (26)], which gives $0.06 \mathrm{meV}$ when evaluated for $d=0.21 \mathrm{meV}$ and $\tilde{\Delta}=0.34 \mathrm{meV}$.

The higher lines plotted in Fig. 12 correspond to transitions into spin- $s_{f}$ state of the form $\left|s_{f}, k\right\rangle$ [cf. Eq. (34) and Fig. 8(d)] (for simplicity these were the only ones considered in [18, 19, 42], though in general others are expected to occur too). The jumps in these lines, e.g. in Fig. 12(a) at $h_{1,2}$, occur whenever the two final excited states $\left|s_{f}, k_{f}\right\rangle$ and $\left|s_{f^{\prime}}, k_{f^{\prime}}\right\rangle$ before and after the GSC at $h_{s_{i}, s_{i}^{\prime}}$ have different correlation energies. (Recall that the correlation energy of an excited state $\left|s_{f}, B_{f}\right\rangle$ can be nonzero even if that of the corresponding ground state $\left|s_{f}\right\rangle$ is zero, since the former's unpaired electrons are further away from $\varepsilon_{\mathrm{F}}$, so that $\Delta_{s_{f}, B_{f}}>\Delta_{s_{f}}$, see point (vi) of Sec. G.) Experimentally, these jumps have not been observed. This may be because up-moving resonances lose amplitude and are difficult to follow [7] with increasing $h$, or because the widths of the excited resonances $(\simeq 0.13 \tilde{\Delta})$ limit energy resolution $[8,9,10]$. 
For somewhat larger grains, the present theory predicts jumps even in the lowest line, as illustrated in Fig. 12(b). It remains to be investigated, though, whether orbital effects, which rapidly increase with the grain size, would not smooth out such jumps.

To conclude this section, we emphasize once again that more than qualitative agreement between theory and experiment can not be expected, since both the model and our variational treatment thereof are very crude: the model neglects, for instance, fluctuations in level spacing and in pair-coupling constants, and the g.c. wave functions become unreliable for $d / \tilde{\Delta} \gtrsim 0.5$. Furthermore, we neglected nonequilibrium effects in the tunneling process and assumed equal tunneling matrix elements for all processes. In reality, though, random variations of tunneling matrix elements could suppress some tunneling processes which would otherwise be expected theoretically.

\section{Measurable consequences of the blocking effect: parity effects}

This section is devoted to various measurable manifestations of the blocking effect, in the form of parity effects, i.e. differences between a grain with an even or odd number of electrons.

\subsection{Bulk consequences of blocking}

The most obvious measurable manifestation of the blocking effect is the very existence of a spectral gap: "breaking a pair" and placing the two newly unpaired electrons in two singly-occupied levels costs a significant amount of correlation energy, because the unpaired electrons loose pairing energy themselves and also disrupt the pairing correlations of the other pairs. This, of course, is already present in standard bulk mean-field BCS theory via the energy cost of at least $2 \tilde{\Delta}$ involved in creating two quasiparticles, and is one of the hallmarks of superconductivity.

In the context of ultrasmall grains, let us denote the pair-breaking energies for an even (odd) grain, i.e. the minimum energy cost per electron for breaking a pair by flipping a single spin at $h=0$, by $\Omega_{e}\left(\Omega_{o}\right)$ :

$$
\Omega_{e} \equiv \frac{1}{2}\left(\mathcal{E}_{1}-\mathcal{E}_{0}\right)_{h=0}, \quad \Omega_{o} \equiv \frac{1}{2}\left(\mathcal{E}_{3 / 2}-\mathcal{E}_{1 / 2}\right)_{h=0} .
$$

The even pair-breaking gap $\Omega_{e}$ is of course strikingly visible in RBT's $h=0$ spectra as a large spectral gap for even grains [cf. Figs. 3 and 5 ; the latter gives $\Omega_{e}=0.26 \mathrm{meV}$ ]. Its presence is direct evidence for the existence of pairing correlations in the grain, which in that sense can still be called "superconducting".

In contrast, the odd pair-breaking gap $\Omega_{o}$ can not be obtained from $h=0$ spectra, since in an odd grain the lowest excitation does not involve breaking a pair, but simply exciting the unpaired electron, which does not require a correlation-induced gap to be overcome. To measure $\Omega_{o}$, a finite field is needed: by Eq. (41), $\Omega_{e}=h_{0,1}$ and $\Omega_{o}=h_{1 / 2,3 / 2}$, hence both spin-flip gaps are equal to level-crossing fields that can be deduced from $h \neq 0$ data, as explained in Sec. 8. For Fig. 5 this yields $\Omega_{e}=0.23 \pm 0.01 \mathrm{meV}$ and $\Omega_{o}=0.24 \pm 0.01 \mathrm{meV}$ [a result further discussed in Sec. 9.4]. The reason that the $\Omega_{e}$-value determined in this way is somewhat smaller than the above-mentioned $0.26 \mathrm{meV}$ determined at $h=0$ is presumably that the experimental 
spectral lines are not perfectly linear in $h$ (having a small $h^{2}$-contribution due to orbital diamagnetism, which should cause the spectroscopic gap to decrease faster with $h$ than in our model).

Another consequence of the blocking effect is that the condensation energies $E_{p / 2}^{\text {cond }}=$ $\mathcal{E}_{p / 2}-\mathcal{E}_{p / 2}^{0}$ for an even and odd grain differ: the unpaired electron of an odd grain weakens its pairing correlations relative to an even grain, so that $E_{1 / 2}^{\text {cond }}$ is less negative than $E_{0}^{\text {cond }}$. In the bulk limit their difference approaches $E_{1 / 2}^{\text {cond }}-E_{0}^{\text {cond }} \rightarrow \tilde{\Delta}$, the energy of a single quasiparticle. For large mesoscopic islands (with $d / \tilde{\Delta} \ll 1$ ) this energy difference has indeed been directly observed: it causes a change from $e$ - to $2 e$-periodicity in the gate-voltage dependence of Coulomb oscillations $533,82,83,84,85,86]$. For ultrasmall grains, however, ground state energy differences are currently not directly measurable, due to experimental difficulties/ explained in detail in Sec. 2.4.6 of Ref. [16].

The parity effects discussed above survive in the bulk limit. Let us now turn to parity effects that result from even-odd differences in the $d$-dependence of various quantities.

\subsection{Parity-dependent pairing parameters}

As is evident from Fig. 9(a,b), not only the condensation energies $E_{p / 2}$ are parity dependent; as soon as one leaves the bulk regime, the pairing parameters $\Delta_{p / 2}$ become parity-dependent too, with $\Delta_{0}>\Delta_{1 / 2}$. In the context of ultrasmall grains this was first emphasized by von Delft et al. [17, but it had been anticipated before by Janko, Smith and Ambegaokar [79] and Golubev and Zaikin [87, who had studied the first correction to the bulk limit, finding $\Delta_{0}-\Delta_{1 / 2}=d / 2$ to leading order in $d / \tilde{\Delta}$; and this result, in turn, had already been published by Soloviev in the nuclear physics literature as long ago as 1961 [74.

The g.c. results of Fig. 9(a), in particular the fact that the critical level spacing $d_{p / 2}^{\mathrm{BCS}}$ at which $\Delta_{p / 2}$ vanishes is smaller for odd than even grains $\left(d_{1 / 2}^{\mathrm{BCS}}<d_{0}^{\mathrm{BCS}}\right)$, suggest that "pairing correlations break down sooner in odd than even grains" [17. However, it should be remembered that the vanishing of $\Delta_{p / 2}$ signals the breakdown of the g.c. approach. A more accurate statement, that is born out by the canonical calculations reviewed in Sec. 9.4, is that the inequality $\mathcal{E}_{1 / 2}>\mathcal{E}_{0}$ persists for arbitrarily large $d$ (see Fig. 14 in Sec. 11), i.e. pairing correlations are always weaker for odd than even grains, although they never vanish altogether in either.

\subsection{Matveev-Larkin parity parameter}

To capture the difference between correlations in even and odd grains in terms of measurable quantities (which $\Delta_{p / 2}$ are not), Matveev and Larkin 24] proposed the parameter (sometimes called "pairing energy" in nuclear physics 46 )

$$
\Delta_{\mathrm{P}}^{\mathrm{ML}} \equiv \mathcal{E}_{1 / 2}^{N+1}-\frac{1}{2}\left(\mathcal{E}_{0}^{N}+\mathcal{E}_{0}^{N+2}\right) \quad \text { (where } N \text { is even) },
$$

\footnotetext{
${ }^{\mathrm{i}}$ In brief: when $V_{\mathrm{g}}$ is changed over a sufficiently large range to see Coulomb oscillations $\left(\sim e / C_{\mathrm{g}} \simeq\right.$ $1 \mathrm{~V}$ ), sudden rigid shifts in the background off-set charge $Q_{0}$ are encountered at random values of $V_{\mathrm{g}}$, which spoil the $2 e$-periodicity which would have been expected otherwise.
} 

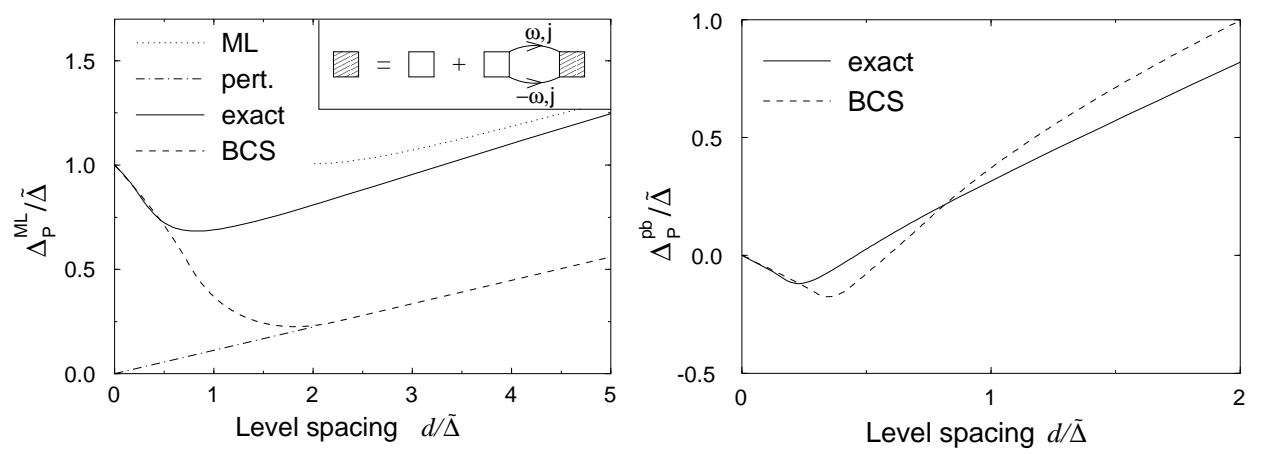

Fig. 13 The parity parameters (a) $\Delta_{\mathrm{P}}^{\mathrm{ML}}$ of Matveev-Larkin [Eq. 443)] and (b) $\Delta_{\mathrm{P}}^{\mathrm{pb}}$ for the pair-breaking energies [Eq. (48)], as functions of $d / \tilde{\Delta}$, calculated using the g.c. variational BCS approach of Sec. 6.1 (dashed lines), and Richardson's exact solution of Sec. 10 (solid lines). In (a), we also show the perturbative result for the uncorrelated Fermi sea, $\left(\Delta_{\mathrm{P}}^{\mathrm{ML}}\right)_{\text {pert }}=$ $\frac{1}{2} \lambda d$ (straight dash-dotted line); and the renormalized result $\left(\Delta_{\mathrm{P}}^{\mathrm{ML}}\right)_{\text {ren }} \simeq d /[2 \ln (a d / \tilde{\Delta})]$ of Eq. (47), in its range of validity $d / \tilde{\Delta} \gg 1$ (dotted line). The parameter $a=1.35$ is chosen to ensure quantitative agreement with the exact result in the limit $d / \tilde{\Delta} \gg 1$. For a summary of the results of various other canonical calculations of $\Delta_{\mathrm{P}}^{\mathrm{ML}}$, see Fig. 14. The inset of (a) shows the Dyson equation used to calculated the renormalized coupling $\lambda$ in Eq. (45).

i.e. the difference between the ground state energy of an odd grain and the mean of the ground state energies of the even grains obtained by removing or adding one electron. Fig. 13 (a) shows its behavior as function of $d / \tilde{\Delta}$. In the bulk limit we have $\mathcal{E}_{0}^{N} \simeq \mathcal{E}_{0}^{N+2}$ and $\Delta_{\mathrm{P}}^{\mathrm{ML}} \simeq \tilde{\Delta}$, which is simply the energy cost for having an unpaired electron on the odd grain. With increasing $d / \tilde{\Delta}$, this energy cost decreases since pairing correlations get weaker, hence $\Delta_{\mathrm{P}}^{\mathrm{ML}}$ initially decreases. It begins to increase again for $d \gtrsim \tilde{\Delta}$, since then pairing correlations are so weak that the behavior of $\Delta_{\mathrm{P}}^{\mathrm{ML}}$ is governed by the "self-energy" of the one extra pair in $\mathcal{E}_{0}^{N+2}$ relative to $\mathcal{E}_{0}^{N}$. For example, in the g.c. variational $\mathrm{BCS}$ result for $\Delta_{\mathrm{P}}^{\mathrm{ML}}$, namely

$$
\left(\Delta_{\mathrm{P}}^{\mathrm{ML}}\right)_{\mathrm{BCS}}=\mathcal{E}_{1 / 2}^{\mathrm{BCS}}-\mathcal{E}_{0}^{\mathrm{BCS}}+\lambda d / 2
$$

it is this self-energy which produces the $\lambda d / 2$ contribution.

A more careful calculation for the regime $d \gg \tilde{\Delta}$ was performed by Matveev and Larkin [24], who considered the renormalization of $\lambda$ due to "pairing fluctuations" about the uncorrelated Fermi ground state $|p / 2\rangle_{0}$. Summing up the leading logarithmic vertex corrections [88] [see inset of Fig. 13(a)], they obtained a renormalized coupling $\tilde{\lambda}$ given, with logarithmic accuracy, by

$$
\begin{aligned}
& \tilde{\lambda}=\lambda+\lambda d\left[\sum_{j}^{U} \int \frac{\mathrm{d} \omega}{2 \pi} \frac{1}{\left[\mathrm{i} \omega-\left(\varepsilon_{j}-\mu\right)\right]} \frac{1}{\left[-\mathrm{i} \omega-\left(\varepsilon_{j}-\mu\right)\right]}\right] \tilde{\lambda} \\
& \tilde{\lambda}=\frac{\lambda}{1-\lambda d \sum_{j}^{U} \frac{1}{2\left|\varepsilon_{j}-\mu\right|}} \simeq \frac{\lambda}{1-\lambda \log \left(\omega_{\mathrm{D}} / d\right)} .
\end{aligned}
$$


This result evidently is valid only if $d \gg \omega_{\mathrm{D}} \mathrm{e}^{-1 / \lambda} \simeq \tilde{\Delta} / 2$ (which, incidentally, is another way of seeing that $d \simeq \tilde{\Delta}$ defines the crossover between the fluctuationdominated and bulk regimes). Matveev and Larkin concluded that

$$
\left(\Delta_{\mathrm{P}}^{\mathrm{ML}}\right)_{\mathrm{ren}} \simeq \tilde{\lambda} d / 2=d /(2 \log d / \tilde{\Delta}) \quad \text { for } \quad d \gg \tilde{\Delta} .
$$

This logarithmic renormalization is beyond the reach of the g.c. variational BCS method, but was confirmed using exact methods [29, 42, 35] (see Sec. 11). Its occurrence, in a regime that in g.c. variational calculations appears to be "uncorrelated", can be regarded as the "first sign of pairing correlations", in particular since, by Eq. (46), the interaction strength increases upon renormalization only if the interaction is attractive $(\lambda<0$ would imply $|\tilde{\lambda}|<|\lambda|)$. The pairing fluctuations responsible for this renormalization will be discussed in more detail in Secs. 11 and 12.

Unfortunately, $\Delta_{\mathrm{P}}^{\mathrm{ML}}$ is at present not measurable in ultrasmall grains, for the same experimental reasons as apply to $\mathcal{E}_{1 / 2}-\mathcal{E}_{0}$ (see footnote $\mathrm{i}$ in Sec. 9.1).

\subsection{Parity effect for pairbreaking energies}

Braun and von Delft [31, 19, 42] discussed yet another parity effect, based on

$$
\Delta_{\mathrm{P}}^{\mathrm{pb}}=\Omega_{o}-\Omega_{e},
$$

the difference between the pair-breaking energies of an even and an odd grain [see Eq. (42)]. Fig. 13 (b) shows its behavior as function of $d$. In the bulk limit $\Omega_{e} \simeq \Omega_{o} \simeq \tilde{\Delta}$ and $\Delta_{\mathrm{P}}^{\mathrm{pb}} \simeq 0$. The most interesting feature of $\Delta_{\mathrm{P}}^{\mathrm{pb}}$ is that it initially becomes negative as $d / \tilde{\Delta}$ increases; this occurs because in an odd grain pairing correlations are weaker and hence breaking a pair costs less energy than in an even grain. $\Delta_{\mathrm{P}}^{\mathrm{pb}}$ becomes positive again for $d / \tilde{\Delta} \gtrsim 0.5$, since then pairing correlations are so weak that $\Delta_{\mathrm{P}}^{\mathrm{pb}}$ is governed by the kinetic energy cost of flipping a spin, which is $2 d$ for an odd grain but only $d$ for an even grain.

$\Delta_{\mathrm{P}}^{\mathrm{pb}}$ is directly measurable in RBT's grains, via the level-crossing fields $h_{0,1}=\Omega_{e}$ and $h_{1 / 2,3 / 2}=\Omega_{o}$ [Eq. (41)]. The measured values $\Omega_{e}=0.23 \pm 0.01 \mathrm{meV}$ and $\Omega_{o}=0.24 \pm 0.01 \mathrm{meV}$ cited in Sec. 9.1 give a positive value of $\Delta_{\mathrm{P}}^{\mathrm{pb}}=0.1 \mathrm{meV}$, implying that the grain under study was too small to fall in the most interesting regime where $\Delta_{\mathrm{P}}^{\mathrm{pb}}$ is negative. Braun and von Delft suggested that the latter should be observable in a somewhat larger grain with $h_{1 / 2,3 / 2}<h_{0,1}$, i.e. in Regime II of Fig. 11. (This suggestion assumes that despite the increased grain size, the complicating effect of orbital diamagnetism is still non-dominant in Regime II.) To look for negative $\Delta_{\mathrm{P}}^{\mathrm{pb}}$ experimentally would thus require good control of the ratio d/ $\tilde{\Delta}$, i.e. grain size. This might be achievable if recently-reported new fabrication methods, which allow systematic control of grain sizes by using colloidal chemistry techniques [89, 90, 91], could be applied to Al grains. 


\section{Part II: Crossover from the bulk to the limit of a few electrons}

Part II of this review is devoted to the question: How do pairing correlations change when the size of a superconductor is decreased from the bulk to the limit of only a few electrons? In particular, we shall attempt to refine the answer given by Anderson [41], namely that superconductivity as we know it breaks down for $d \gtrsim \tilde{\Delta}$.

First steps towards a more detailed answer were taken in the early 1970s by Strongin et al. [61] and by Mühlschlegel et al. [62], who calculated the thermodynamic properties of ensembles small superconducting grains. Experimental realizations of such ensembles were, e.g., the granular films studied by Giaver and Zeller [59, 60]. The interest of theorists was rekindled in 1995 by RBT's success in probing individual superconducting grains. Apart from motivating the phenomenological theory of Braun et al. reviewed in part I, these experiments also inspired a substantial and still growing number of theoretical studies [17]- 40 of how superconducting pairing correlations in such grains are affected by reducing the grains' size, or equivalently by increasing its mean level spacing $d \propto \mathrm{Vol}^{-1}$ until it exceeds the bulk gap $\tilde{\Delta}$.

In the earliest of these, von Delft et al. studied the discrete BCS model of Sec. A within a parity-projected g.c. BCS approach [17] closely related to the variational BCS method of Sec. 6. Their g.c. results suggested that pairing correlations, as measured by the pairing parameter or the condensation energy, vanish abruptly once $d$ exceeds a critical level spacing $d_{p / 2}^{\mathrm{BCS}}$ that depends on the parity $(p=0$ or 1$)$ of the number of electrons on the grain, being smaller for odd grains $\left(d_{1 / 2}^{\mathrm{BCS}} \simeq 0.89 \tilde{\Delta}\right)$ than even grains $\left(d_{0}^{\mathrm{BCS}} \simeq 3.6 \tilde{\Delta}\right)$. Parity effects were also found in a number of subsequent papers that used parity-projected g.c. methods to study the behavior of the BCS mean-field gap parameter $\Delta_{\mathrm{gc}}$ and related quantities as functions of level spacing [18][28], temperature and magnetic field. All these parity effects are consequences of the blocking effect (cf. Sec. 4.4): for odd grains, the unpaired electron somewhat disrupts the pairing correlations of the remaining paired ones, by reducing the phase space available for pair scattering.

A series of more sophisticated canonical approaches [29- 37] (summarized in Sec. 11) confirmed the parity dependence of pairing correlations, but established that the abrupt vanishing of pairing correlations at $d_{p / 2}^{\mathrm{BCS}}$ is an artifact of g.c. treatments: pairing correlations do persist, in the form of so-called fluctuations, to arbitrarily large level spacings [24, and the crossover between the bulk superconducting (SC) regime $(d \ll \tilde{\Delta})$ and the fluctuation-dominated (FD) regime $(d \gg \tilde{\Delta})$ is completely smooth [32]-[36]. Nevertheless, these two regimes are qualitatively very different [31]-[36]: the condensation energy, e.g., is an extensive function of volume in the former and almost intensive in the latter, and pairing correlations are quite strongly localized around the Fermi energy $\varepsilon_{\mathrm{F}}$, or more spread out in energy, respectively. Very recently, Di Lorenzo et al. [40] suggested that the remnant pairing correlations in the FD regime might be detectable via susceptibility measurements.

Toward the end of 1998 and after the appearance of most of these works, R.W. Richardson pointed out 92] to their various authors that the discrete BCS Hamiltonian on which they are based actually has an exact solution, discovered by him in 1963 [43 (and independently by Gaudin in 1968 [93). Richardson published his solution in the 
context of nuclear physics in a series of papers between 1963 and 1977 [43]- 51] which seem to have completely escaped the attention of the condensed matter community. Very recently, the model was also shown to be integrable [94, 95. The revival of this remarkably simple exact solution after such a long and undeserved period of neglect is perhaps one of the most important consequences of RBT's experimental breakthrough: Richardson's solution allows the elucidation and illustration by exact means of many important conceptual ingredients of the standard BCS theory of superconductivity, such as the nature of pairing correlations, the importance of phase coherence, the validity of using a mean-field approximation and a grand-canonical formulation for bulk systems, and the limitations of the latter approaches for ultrasmall systems. Moreover, it allows the exact calculation of essentially all quantities of interest for ultrasmall grains.

We shall therefore start part II by discussing the exact solution [Sec. 10]. We then summarize the other canonical approaches somewhat more briefly than they perhaps would have deserved had an exact solution not existed, and compare their results to those of the exact solution [Sec. 11]. Next we analyze the qualitative differences between the bulk and FD regimes [Sec. 12], then discuss the case of randomly (as opposed to uniformly) spaced energy levels $\varepsilon_{j}$ [Sec. 13], and finally discuss finite temperature parity effects [Sec. 14]. Throughout part II we set $\mu=0$, since canonical treatments make no reference to a chemical potential.

\section{Richardson's exact solution}

In this section we summarize some of the central results of Richardson's exact solution of the discrete BCS model.

\subsection{General eigenstates}

Consider $N=2 n+b$ electrons, $b$ of which are unpaired, as in Sec. 4.4. According to the general discussion there, the nontrivial aspect of solving the model is finding the eigenenergies $\mathcal{E}_{n}$ and corresponding eigenstates $\left|\Psi_{n}\right\rangle$ [Eq. (13)] of the pair Hamiltonian [Eq. (14), in which we set $\mu=0$ below]

$$
\hat{H}_{U}=\sum_{i j}^{U}\left(2 \varepsilon_{j} \delta_{i j}-\lambda d\right) b_{i}^{\dagger} b_{j}
$$

in the Hilbert space of all states containing exactly $n$ pairs $b_{j}^{\dagger}=c_{j+}^{\dagger} c_{j-}^{\dagger}$ of electrons, where $j$ runs over the set of all unblocked single-particle levels, $U=I \backslash B[I$ is the set of all interacting levels, $B$ the set of all blocked levels]. In general, degenerate levels are allowed in $I$, but are to be distinguished by distinct $j$-labels, i.e. they have $\varepsilon_{i}=\varepsilon_{j}$ for $i \neq j$.

Richardson showed that the sought-after eigenstates (with normalization $\left\langle\Psi_{n} \mid \Psi_{n}\right\rangle=1$ ) and eigenenergies have the general form

$$
\left|\Psi_{n}\right\rangle=\mathcal{N} \prod_{\nu=1}^{n} B_{\nu}^{\dagger}|0\rangle, \quad \mathcal{E}_{n}=\sum_{\nu=1}^{n} E_{\nu}, \quad \text { with } \quad B_{\nu}^{\dagger}=\sum_{j}^{U} \frac{b_{j}^{\dagger}}{2 \varepsilon_{j}-E_{\nu}} .
$$


Here $\mathcal{N}$ is a normalization constant and the $n$ parameters $E_{\nu}(\nu=1, \ldots, n)$ are a solution of the set of $n$ coupled algebraic equations

$$
\frac{1}{\lambda d}-\sum_{j}^{U} \frac{1}{2 \varepsilon_{j}-E_{\nu}}+\sum_{\mu=1(\neq \nu)}^{n} \frac{2}{E_{\mu}-E_{\nu}}=0, \quad \text { for } \quad \nu=1, \ldots, n
$$

which are to be solved (numerically, see App. B2 of Ref. [16]) subject to the restrictions $E_{\mu} \neq E_{\nu}$ if $\mu \neq \nu$. Richardson originally derived this remarkably simple result by solving the Schrödinger equation for the wave-function $\psi\left(j_{1}, \ldots, j_{n}\right)$ of Eq. (11). A simpler proof, also due to Richardson [96], may be found in Ref. 36] and in App. B.1 of Ref. [16]; its strategy is to verify that $\left(\hat{H}_{U}-\mathcal{E}_{n}\right)\left|\Psi_{n}\right\rangle=0$ by simply commuting $\hat{H}_{U}$ past the $B_{\nu}^{\dagger}$ operators in $(50)$.

Below we shall always assume the $\varepsilon_{j}$ 's to be all distinct (the more general case that degeneracies are present is discussed by Gaudin [93]). Then it can be shown explicitly [93 that (i) the number of distinct solutions of Eq. (51) is equal to the dimension of the $n$-pair Hilbert space defined on the set of unblocked levels $U$, namely $\left(\begin{array}{c}N_{U} \\ n\end{array}\right)$, where $N_{U}$ is the number of unblocked levels; and (ii) that the corresponding eigenstates (50) are mutually orthogonal to each other, thus forming an eigenbasis for this Hilbert space. This can easily be understood intuitively, since there exists a simple relation between the bare pair energies $2 \varepsilon_{j}$ and the solutions of Eqs. (51): as $\lambda$ is reduced to 0, it follows by inspection that each solution $\left\{E_{1}, \ldots, E_{n}\right\}$ reduces smoothly to a certain set of $n$ bare pair energies, say $\left\{2 \varepsilon_{j_{1}}, \ldots, 2 \varepsilon_{j_{n}}\right\}$; this particular solution may thus be labeled by the indices $j_{1}, \ldots, j_{n}$, and the corresponding eigenstate (50) written as $\left|\Psi_{n}\right\rangle \equiv$ $\left|j_{1}, \ldots j_{n}\right\rangle$. By inspection, its $\lambda \rightarrow 0$ limit is the state $\left|j_{1}, \ldots j_{n}\right\rangle_{U, 0} \equiv \prod_{\nu=1}^{n} b_{j_{\nu}}^{\dagger}|0\rangle$, thus there is a one-to-one correspondence between the sets of all states $\left\{\left|j_{1}, \ldots, j_{n}\right\rangle_{U}\right\}$ and $\left\{\left|j_{1}, \ldots j_{n}\right\rangle_{U, 0}\right\}$. But the latter constitute a complete eigenbasis for the $n$-pair Hilbert space defined on the set of unblocked levels $U$, thus the former do too.

\subsection{Ground state}

For a given set of blocked levels $B$, the lowest-lying of all states $\left|\Psi_{n}, B\right\rangle$ of the form (10), say $\left|\Psi_{n}, B\right\rangle_{\mathrm{G}}$, is obtained by using that particular solution $\left|j_{1}, \ldots j_{n}\right\rangle$ for which the total "pair energy" $\mathcal{E}_{n}$ takes its lowest possible value. The lowest-lying of all eigenstates with $n$ pairs, $b$ blocked levels and total spin $s=b / 2$, say $|n, s\rangle_{\mathrm{G}}$ with energy $\mathcal{E}_{s}^{\mathrm{G}}(n)$, is that $\left|\Psi_{n}, B\right\rangle_{\mathrm{G}}$ for which the blocked levels in $B$ all contain spin-up electrons and are all as close as possible to $\varepsilon_{\mathrm{F}}$, the Fermi energy of the uncorrelated $N$-electron Fermi sea $\left|\mathrm{F}_{N}\right\rangle$. The $E_{\nu}$ for the ground state $|n, s\rangle_{\mathrm{G}}$ coincide at $\lambda=0$ with the lowest $n$ energies $2 \varepsilon_{j}(j=1, \ldots, n)$, and smoothly evolve toward (initially) lower values when $\lambda$ is turned on, a fact that can be exploited during the numerical solution of Eq. (51). As $\lambda$ is increased further, some of the $E_{\nu}$ 's become complex; however, they always occur in complex conjugate pairs, so that $\mathcal{E}_{n}$ remains real 48]. For details, see Ref. 48] and App. B.2 of Ref. [16], where some algebraic transformations are introduced that render the equations less singular and hence simplify their numerical solution considerably. 


\subsection{General comments}

Since the exact solution provides us with wave functions, it is in principle straightforward to calculate arbitrary correlation functions of the form $\left\langle\Psi_{n}\left|b_{i}^{\dagger} b_{j}^{\dagger} \ldots b_{i^{\prime}} b_{j^{\prime}}\right| \Psi_{n}\right\rangle$, by simply commuting all $b$ 's to the right of all $b^{\dagger}$ 's. However, due to the hard-core boson commutation relations (16) of the $b$ 's, the combinatorics is rather involved. Nevertheless, Richardson succeeded to derive [47] explicit results for the normalization constant $\mathcal{N}$ of (50) and the occupation probabilities $\bar{v}_{j}^{2}$ and correlators $C_{i j}$ of Eq. (19) (summarized in App. B.3 of Ref. [16]). The exact result for the $C_{i j}$ 's show that they are all positive, in agreement with the requirement (ii) formulated in Sec. 5.2. It is also natural to ask whether in the bulk limit $(d \rightarrow 0$ at fixed $n d)$, the standard BCS results can be extracted from the exact solution. Indeed they can, as Richardson showed in [51] (following unpublished work by Gaudin 93]), by interpreting the problem of solving the eigenvalue equations (51) for the $E_{\nu}$ as a problem in two-dimensional electrostatics (see App. B.2 of Ref. [16]). Exploiting this analogy, he showed that in the bulk limit, Eqs. (51) reduce to the well-known BCS equations determining the gap and chemical potential at $T=0$ [Eqs. (38) and (39)], and the ground state condensation energy $\mathcal{E}_{0}^{\text {cond }}(n)[$ Eq. 150$]$ to its BCS result, namely $-\tilde{\Delta}^{2} / 2 d$.

Finally, let us mention that the Cambiaggio, Rivas and Saraceno have recently shown that the discrete BCS model is integrable and have constructed explicit expressions for all its constants of the motion [94]. The latter's relation to Richardson's solution was clarified by Sierra [95], who has also explored possible connections between the exact solution and conformal field theory. It would be an interesting challenge for mathematical physicists to try to exploit this integrability to calculate finitetemperature properties exactly - although these can in principle be obtained from Richardson's solution by "simply" computing the partition function over all states, this is forbiddingly tedious in practice for large temperatures, since the eigenenergy of each state requires a separate (non-trivial) numerical calculation.

\section{Comparison of other canonical methods with the exact solution}

In this section we briefly mention the various canonical methods by which the discrete BCS model had been investigated prior to the revival of Richardson's exact solution in 1999. All of these studies used a half-filled band with fixed width $2 \omega_{\mathrm{D}}$ of uniformlyspaced levels [i.e. $\varepsilon_{j}=j d+(1-p) d / 2$, as in Eq. (7)], containing $N=2 n+b$ electrons. Then the level spacing is $d=2 \omega_{\mathrm{D}} / N$ and the bulk gap is $\tilde{\Delta}=\omega_{\mathrm{D}} / \sinh (1 / \lambda)$. Following [31], we take $\lambda=0.224$ throughout this section. To judge the quality of the various approaches, we compare in Fig. 14 the results which they yield with those from Richardson's solution, for the even and odd $(s=0,1 / 2)$ condensation energies $E_{s}^{\text {cond }}$ and the Matveev-Larkin parity parameter $\Delta_{\mathrm{P}}^{\mathrm{ML}}[\mathrm{cf}$. Sec. 9.3]. In the notation of Sec. 10.2, these are given by

$$
\begin{aligned}
E_{s}^{\text {cond }}(n) & =\mathcal{E}_{s}^{\mathrm{G}}(n)-\left\langle\mathrm{F}_{N}|\hat{H}| \mathrm{F}_{N}\right\rangle, \\
\Delta_{\mathrm{P}}^{\mathrm{ML}}(n) & =\mathcal{E}_{1 / 2}^{\mathrm{G}}(n)-\left[\mathcal{E}_{0}^{\mathrm{G}}(n)+\mathcal{E}_{0}^{\mathrm{G}}(n+1)\right] / 2 .
\end{aligned}
$$

Following the initial g.c. studies [17, 18, 19, 20, 24] of the discrete BCS model, the 
first purely canonical study was that of Mastellone, Falci and Fazio (MFF) [29], who used Lanczos exact diagonalization. Despite being limited to $n \leq 12$, they managed to reach reasonably small ratios of $d / \tilde{\Delta}$ by using an ingenious scaling approach: for a given level spacing $d$, they increased the coupling constant $\lambda$ to about 0.5 , thereby decreasing $d / \tilde{\Delta}=d / \omega_{\mathrm{D}} \sinh (1 / \lambda)$ to values as small as 0.5 . This allowed them to probe, coming from the few-electron side, a remarkably large part of the crossover to bulk limit. They found, i.a., that the condensation energies are negative for all $d$, showing that the system can always gain energy by allowing pairing correlations, even for arbitrarily large $d$.

Berger and Halperin (BH) [30] showed that almost identical results can be achieved with less than 6 pairs, thus significantly reducing the calculational effort involved, by first performing a "poor man's scaling" renormalization: they reduce the bandwidth from $\omega_{\mathrm{D}} \approx n d$ to, say, $\bar{\omega}_{\mathrm{D}} \approx \bar{n} d$ (with $\bar{n} \leq 6$ ) and incorporate the effect of the removed levels by using a renormalized coupling constant,

$$
\bar{\lambda}=\lambda\left[1-\sum_{\bar{\omega}_{\mathrm{D}}<\left|\varepsilon_{j}\right|<\omega_{\mathrm{D}}} \frac{\lambda}{2\left|\varepsilon_{j}\right|}\right]^{-1} .
$$

The reduced system is then diagonalized exactly. Note that the renormalization of Matveev and Larkin [Eq. (46)] corresponds to taking $\bar{\omega}_{\mathrm{D}} \simeq d$ in Eq. (54), i.e. to integrating out the entire band. Also note that the renormalization prescription of (54) has the property that it would leave the bulk gap invariant in the limit $d / \tilde{\Delta} \rightarrow 0$, for which Eq. (54) would imply $\bar{\omega}_{\mathrm{D}} e^{-1 / \bar{\lambda}} \simeq \omega_{\mathrm{D}} e^{-1 / \lambda} \simeq \tilde{\Delta}$.

To access larger values of $n$, Braun and von Delft [31] used a fixed-n projected BCS approach (PBCS), in which BCS-like variational wavefunctions are projected to fixed particle number, as in Eq. (30). The projection integrals occurring in Eq. (30) were evaluated numerically for $n \leq 600$, using tricks developed in the nuclear physics literature by Bayman [97], Dietrich, Mang and Pradal [98] and Ma and Rasmussen [99], and summarized in part in the book of Ring and Schuck [76]. (A much simpler way of dealing with the projection, using recursion relations, was recently found by Dukelsky and Sierra [33].) The PBCS method gives condensation energies that (i) are significantly lower than the grand-canonical ones [see Fig. 14], thus the projection much improves the variational Ansatz, and that (ii) are negative for all $d$, confirming that the abrupt vanishing of the g.c. condensation energies is indeed an artifact of the g.c. treatment. The PBCS method is able to fully recover the bulk limit, but the crossover is not completely smooth and shows a remnant of the g.c. breakdown of pairing correlations: the $d$-dependence of the condensation energy $\left(E_{s}^{\text {cond }}\right)^{\mathrm{PBCS}}$ changes rather abruptly [kinks in the short-dashed lines in Fig. 14(a)] from being extensive $(\sim 1 / d)$ to being practically intensive (almost $d$ independent).

It should be mentioned here that a generalization of the PBCS method to finite temperatures has been worked out by Essebag and Egido in the context of nuclear physics 100. The PBCS method has recently also been applied to the attractive Hubbard model in one dimension by Tanaka and Marsiglio [39], who found even-odd and super-even effects. The latter consist of differences between the number of pairs being equal to $n=2 m$ or $2 m+1$, and arise if boundary conditions are used that produce doubly-degenerate levels $\left(\varepsilon_{\vec{k}}=\varepsilon_{-\vec{k}}\right)$ near the Fermi surface. 


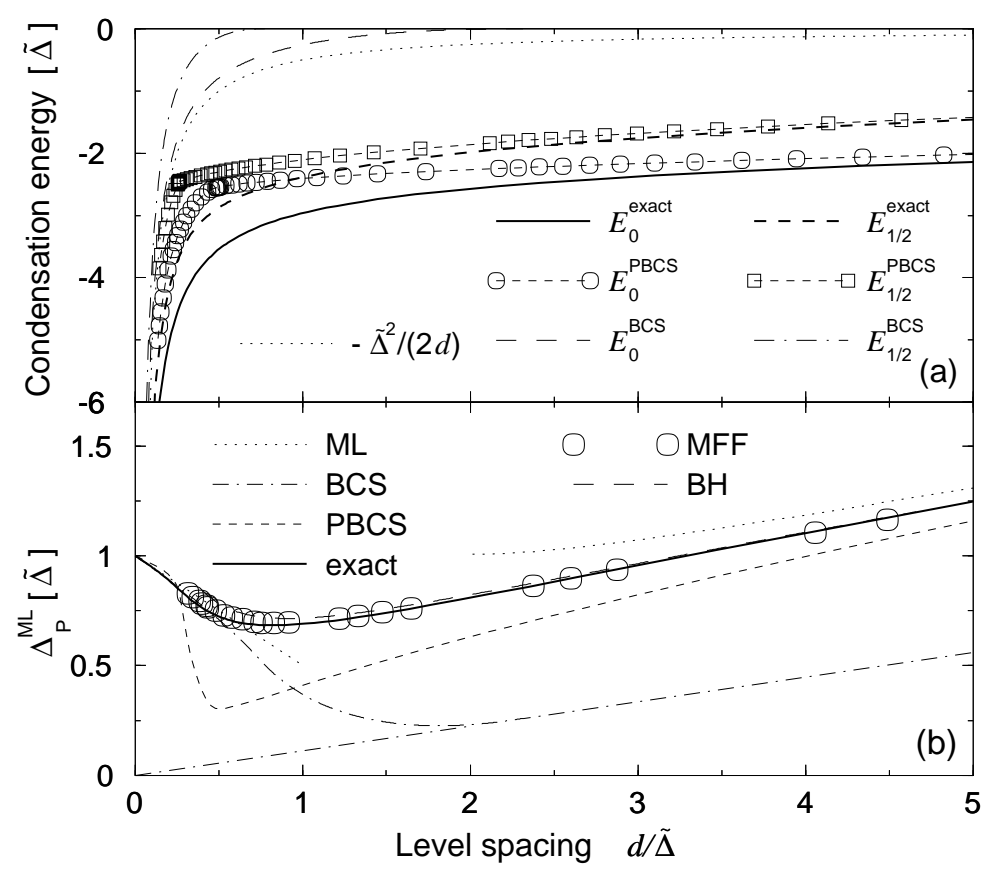

Fig. 14 (a) The even and odd $(s=0,1 / 2)$ condensation energies $E_{s}^{\text {cond }}$ of Eq. (52) [in units of $\tilde{\Delta}$ ], calculated with BCS, PBCS and exact wave functions 35, as functions of $d / \tilde{\Delta}=2 \sinh (1 / \lambda) /(2 n+2 s)$, for $\lambda=0.224$. For comparison, the dotted line gives the "bulk" result $E_{0}^{\text {bulk }}=-\tilde{\Delta}^{2} /(2 d)$. (b) Comparison 35] of the parity parameters $\Delta_{\mathrm{P}}^{\mathrm{ML}}$ [24 of Eq. (53) [in units of $\tilde{\Delta}$ ] obtained by various authors: ML's analytical result (dotted lines) $[\tilde{\Delta}(1-d / 2 \tilde{\Delta})$ for $d \ll \tilde{\Delta}$, and $d / 2 \log (a d / \tilde{\Delta})$ for $d \gg \tilde{\Delta}$, with $a=1.35$ adjusted to give asymptotic agreement with the exact result]; grand-canonical BCS approach (dash-dotted line) [the naive perturbative result $\frac{1}{2} \lambda d$ is continued to the origin]; PBCS approach (shortdashed line); Richardson's exact solution (solid line); exact diagonalization and scaling by MFF (open circles) and BH (long-dashed line).

Dukelsky and Sierra 32, 33] used the density matrix renormalization group (DMRG) (with $n \leq 400$ ) to achieve significant improvements over the PBCS results for the discrete BCS model, in particular in the regime of the crossover, which they found to be completely smooth. In general, the DMRG approach is applicable to systems that can be divided into two pieces, called block and environment, which interact via a preferably rather small number of states. One starts with a small block and environment, computes their combined density matrix, then enlarges both and recomputes the density matrix, etc, until a large part of the system has been treated. Dukelsky and Sierra chose the block and environment to consist, respectively, of all particle or hole states relative to the Fermi sea (for a detailed description of the method, see [33]). Since the pairing correlations involving coherent superpositions of particle and hole states are peaked in a rather small regime of width $\tilde{\Delta}$ around the Fermi energy [compare Figs. 0 or 16], the "interaction" between block and environment is "localized", so that the 
DMRG can a priori be expected to work rather well for this problem.

Finally, Dukelsky and Schuck [37] showed that a self-consistent RPA approach, which in principle can be extended to finite temperatures, describes the FD regime rather well (though not as well as the DMRG).

To check the quality of the above methods, Braun 42, 35] computed $E_{s}^{\text {cond }}$ (for $s=0,1 / 2$ ) and $\Delta_{\mathrm{P}}^{\mathrm{ML}}$ using Richardson's solution (Fig. 14). The exact results

(i) quantitatively agree, for $d \rightarrow 0$, with the leading $-\tilde{\Delta}^{2} / 2 d$ behavior for $E_{s}^{\text {cond }}$ obtained in the g.c. BCS approach [17, 31, 19], which in this sense is exact in the bulk limit, corrections being of order $d^{0}$;

(ii) confirm that the even ground state energy always lies below the odd one (this had independently been proven rigoroulsy by Tian and Tang [38]);

(iii) confirm that a completely smooth [32, 33] crossover occurs around the scale $d \simeq \tilde{\Delta}$ at which the g.c. BCS approach breaks down;

(iv) show that the PBCS crossover [31] is qualitatively correct, but not quantitatively, being somewhat too abrupt;

(v) are reproduced remarkably well by the approaches of MFF [29] and BH [30];

(vi) are fully reproduced by the DMRG of [32, 33] with a relative error of $<10^{-4}$ for $n \leq 400$; our figures don't show DMRG curves, since they are indistinghuishable from the exact ones and are discussed in detail in [32, 33].

The main conclusion we can draw from these comparisons is that the two approaches based on renormalization group ideas work very well: the DMRG is essentially exact for this model, but the band-width rescaling method of $\mathrm{BH}$ also gives remarkably (though not quite as) good results with rather less effort. In contrast, the PBCS approach is rather unreliable in the crossover region. To study generalizations of the discrete BCS model, e.g. using state-dependent couplings of the form $d \sum_{i j} \lambda_{i j} b_{i}^{\dagger} b_{j}$, the DMRG would thus be the method of choice.

\section{Qualitative differences between the bulk and the few-electron regimes}

Does the fact that the exact condensation energy $E_{s}^{\text {cond }}$ is always negative, even for arbitrarily large $d / \tilde{\Delta}$, mean that the system stays "superconducting" even if it is arbitrarily small? The answer is certainly no, since in the fluctuation-dominated (FD) regime, the pairing correlations are qualitatively different than in the bulk, superconducting regime. In this section we shall try to make this statement more precise by analyzing the qualitative differences between the two regimes, with regard to the $\lambda$ and $d$-dependence of $E_{s}^{\text {cond }}$, and the behavior of the occupation probabilities $\bar{v}_{j}^{2}$.

Fig. 15(a) shows, on a log-log plot, the $d$-dependence of the even condensation energy $E_{0}^{\text {cond }}(d)$. Note that even on the log-log plot, the crossover of the exact $E_{0}^{\text {cond }}$ from the bulk to the FD regime is completely smooth. According to Sierra and Dukelsky [33], the exact result for $E_{0}^{\text {cond }}(d)$ can be fitted very well to the form

$$
E_{0}^{\text {cond }}(d)=-\tilde{\Delta}^{2} /(2 d)-\eta_{0}(\ln 2) \omega_{\mathrm{D}} \lambda^{2}+\gamma_{0}\left(\tilde{\Delta} d / 2 \omega_{\mathrm{D}}\right) \log \left(2 \omega_{\mathrm{D}} / d\right),
$$




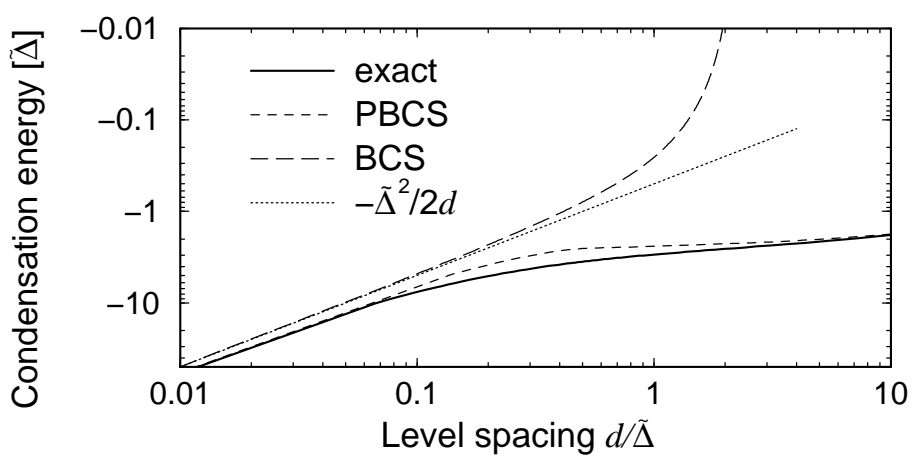

Fig. 15 Log-log plot 42] of some of the curves of Fig. 14(a) for the even condensation energy $E_{0}^{\text {cond }}$ [in units of $\tilde{\Delta}$ ], for $\lambda=0.224$; its asymptotic $-\tilde{\Delta}^{2} /(2 d)$ behavior for $d / \tilde{\Delta} \rightarrow 0$ is shown by the dotted line.

where $\eta_{0}$ and $\gamma_{0}$ are constants of order unity [33. The first term is extensive $(\propto \mathrm{Vol})$ and dominates in the bulk limit; its standard heuristic interpretation [58 is that roughly $\tilde{\Delta} / d$ levels (those within $\tilde{\Delta}$ of $\varepsilon_{\mathrm{F}}$ ) are strongly affected by pairing, with an average energy gain per level of $-\tilde{\Delta} / 2$. The second term, which is intensive and dominates in the FD limit, is equal (up to the numerical factor $\eta_{0}$ ) to the result from second-order perturbation theory [33], namely $(\lambda d)^{2} \sum_{i=1}^{n} \sum_{j=n+1}^{2 n}\left(2 \varepsilon_{i}-2 \varepsilon_{j}\right)^{-1}$. This subleading term's $d$-independence (which was anticipated in [41, 78]) may be interpreted by arguing that in the FD regime, the number of levels that contribute significantly to $E_{0}^{\text {cond }}$ is no longer of order $\tilde{\Delta} / d$ : instead, fluctuations affect all $n \simeq$ $2 \omega_{\mathrm{D}} / d$ unblocked levels within $\omega_{\mathrm{D}}$ of $\varepsilon_{\mathrm{F}}$ (this is made more precise below), and each of these levels contributes an amount of order $-(\lambda d)^{2} / d$ (corresponding, in a way, to its selfenergy). Finally, the third term contains the small parameter $\tilde{\Delta} / \omega_{\mathrm{D}}$ and thus represents a very small correction.

The $\lambda$ - and volume-dependencies of $E_{0}^{\text {cond }}$ in Eq. (55) strikingly illustrate the qualitative differences between the bulk and FD regimes: in the bulk regime, dominated by the first term, $E_{0}^{\text {cond }}$ is nonperturbative in $\lambda$ (since $\tilde{\Delta} \simeq 2 \omega_{D} e^{-1 / \lambda}$ ) and extensive, as expected for a strongly-correlated state; in constrast, in the FD regime, dominated by the second term, $E_{0}^{\text {cond }}$ is perturbative in $\lambda$ and practically intensive (up to the weak $\log d$ dependence of the third term).

Perhaps the most vivid way of illustrating the qualitative difference between the bulk and FD regimes is to study properties of the ground state wavefunction. We shall consider here the correlators [31]

$$
\bar{C}_{j}^{2}(d)=\left\langle b_{j}^{\dagger} b_{j}\right\rangle\left\langle b_{j} b_{j}^{\dagger}\right\rangle,
$$

which measure the probability that a level can be "both occupied and empty", and vanish identically for states without pairing correlations. For the discrete BCS model $\bar{C}_{j}^{2}$ identically equals $\left\langle b_{j}^{\dagger} b_{j}\right\rangle-\left\langle b_{j}^{\dagger} b_{j}\right\rangle^{2}=\bar{v}_{j}^{2}-\bar{v}_{j}^{4}$ [by Eqs. (16) and (19)], which measures the fluctuations in the pair occupation number of level $j$, and it vanishes for any 


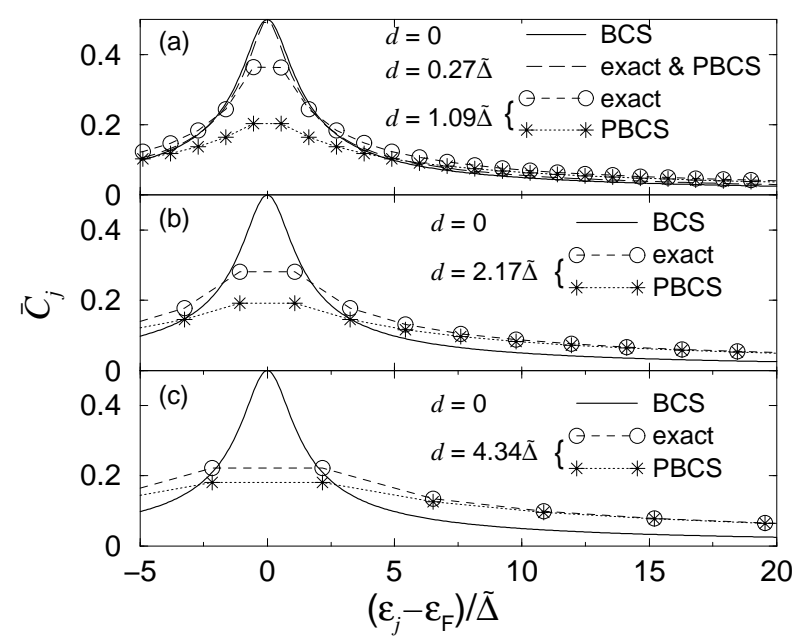

Fig. 16 The occupation probabilities $\bar{C}_{j}$ of Eq. (56) for $d / \tilde{\Delta}=0,0.27,1.09,2.17$ and 4.34 42]. In all three figures, the thick solid lines give the $d=0$ bulk BCS result, whereas circles and stars represent $\bar{C}_{j}$-values evaluated for discrete $j$ 's using the exact solution and PBCS method, respectively. For $d=0.27 \tilde{\Delta}$, the PBCS and exact results are indistinguishable, and are shown in (a) as a single long-dashed line, which is also virtually identical to the bulk curve. For small $d$, pairing correlations are evidently localized within a few $\tilde{\Delta}$ of $\varepsilon_{\mathrm{F}}$. With increasing $d$ more and more weight is shifted away from $\varepsilon_{\mathrm{F}}$ into the tails; compared to the exact results, the PBCS method somewhat overemphasizes this delocalization, which is one of the reasons why it produces a somewhat too abrupt crossover.

blocked single-particle level. Note that $\bar{C}_{j}^{2}(d)$ also equals $\left\langle b_{j}^{\dagger} b_{j}\right\rangle-\left\langle c_{j+}^{\dagger} c_{j+}\right\rangle\left\langle c_{j-}^{\dagger} c_{j-}\right\rangle$; this form, which was used in [31] and corresponds to the diagonal terms under the sum in Eq. (22) for $\Delta_{\text {can }}$, can be interpreted as the probability enhancement for finding a pair of electrons instead of two uncorrelated electrons in a single-particle level $|j, \pm\rangle$.

When evaluated using the grand-canonical BCS wavefunction, $\left(\bar{C}_{j}^{2}\right)_{\text {BCS }}$ is equal to $u_{j}^{2} v_{j}^{2}=\frac{1}{4} \tilde{\Delta}^{2} /\left(\varepsilon_{j}^{2}+\tilde{\Delta}^{2}\right)$ [thick solid lines in Fig. 16, the same function as that plotted in Fig. [1. The $\left(\bar{C}_{j}\right)_{\mathrm{BCS}}$ 's thus have a characteristic peak of width $\propto \tilde{\Delta}$ around $\varepsilon_{\mathrm{F}}$, implying that pairing correlations are "localized around $\varepsilon_{\mathrm{F}}$ in energy space", which may be taken to be the defining property of "BCS-like correlations". Moreover, in the bulk regime $d \ll \tilde{\Delta}$, the $\left(\bar{C}_{j}\right)_{\text {BCS }}$ are virtually identical to $\left(\bar{C}_{j}\right)_{\text {exact }}$ [long-dashed line of Fig. 16(a)], vividly illustrating why the grand-canonical BCS approximation is so successful: not performing a canonical projection hardly affects the parameters $\bar{u}_{j}$ and $\bar{v}_{j}$ if $d \ll \tilde{\Delta}$, but tremendously simplifies their calculation.

As one enters the FD regime $d \gtrsim \tilde{\Delta}$, the character of the correlator $\left(\bar{C}_{j}\right)_{\text {exact }}$ changes [Fig. 16.(b), circles]: weight is shifted into the tails far from $\varepsilon_{\mathrm{F}}$ at the expense of the vicinity of the Fermi energy. Thus pairing correlations become delocalized in energy space (as also found in [29, 32, 33), so that referring to them as mere "fluctuations" is quite appropriate. In the extreme case $d \gg \tilde{\Delta}$, the $\left(\bar{C}_{j}\right)_{\text {exact }}$ for all interacting levels 


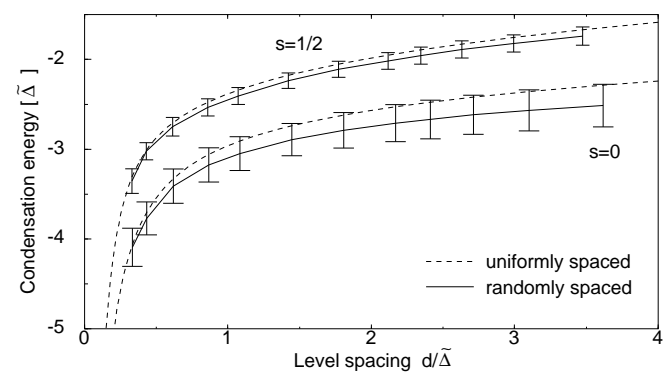

Fig. 17 Exact even and odd condensation energies $E_{s}^{\text {cond }}$ [in units of $\tilde{\Delta}$ ] for equally spaced levels (dashed line), and the ensemble-average $\left\langle E_{s}^{\text {cond }}\right\rangle$ for randomly-spaced levels (solid line) [35]. The height of the fluctuation bars gives the variances $\delta E_{s}^{\text {cond }}$.

are roughly equal.

Richardson's solution can also be used to calculate, for a given set $B$ of blocked levels, the $d$-dependence of the canonical order parameter $\Delta_{\text {can }}^{B}(d)$ of Eq. (22). Schechter has found [101] that it can be fit to the form $\Delta_{\text {can }}^{B}(d)=\tilde{\Delta}\left(1+\tilde{\gamma}_{B} d / \Delta\right)$, where $\tilde{\gamma}_{B}$ is a positive numerical constant, and the linear term essentially reflects the factor of $d$ in the definition of $\Delta_{\text {can }}^{B}$. The fact that $\Delta_{\text {can }}^{B}$ is a strictly increasing function of $d$ is in very striking contrast to the behavior of the grand-canonical pairing parameters $\Delta_{s}(d)$ shown in Fig. 9 (a).

\section{Effect of level statistics}

Smith and Ambegaokar investigated the effect of level statistics on the crossover between the bulk and FD regimes [20]. In contrast to the uniform level spacing used in previous works, they employed a random spacing of levels, distributed according to the gaussian orthogonal ensemble. Using a g.c. mean-field BCS approach, they found, interestingly, that randomness enhances pairing correlations: compared to uniform spacings (u.s.) [17], it (i) on average lowers the condensation energy $E_{s}^{\text {cond }}$ to more negative values, $\left\langle E_{s}^{\text {cond }}\right\rangle<E_{s}^{\text {cond }}$ (u.s.), but (ii) these still are parity dependent, $\left\langle E_{0}^{\text {cond }}\right\rangle\left\langle\left\langle E_{1 / 2}^{\text {cond }}\right\rangle\right.$. These results can readily be understood intuitively: pairing correlations become stronger the higher the density of levels around $\varepsilon_{\mathrm{F}}$, where pair-mixing costs the least energy. When determining the amount of pairing correlations for a set of randomly-spaced levels, fluctuations that increase the level density near $\varepsilon_{\mathrm{F}}$ are thus weighted more than those that decrease it, so that randomness enhances pairing correlations.

Although the g.c. mean-field treatment of Smith and Ambegaokar breaks down for mean level spacings much larger than $\tilde{\Delta}$, just as was the case in [17, 18, 19], their main conclusions (i) and (ii) are robust. Indeed, these were recently confirmed by Sierra et al. [35], who used Richardson's exact solution to calculate $E_{s}^{\text {cond }}$ for ensembles of random levels [Fig. 17]. Moreover, they found that the blocking effect responsible for (ii) manifests itself in the fluctuations too, which likewise are parity dependent: for example, Fig. 17 shows that both the variances $\delta E_{s}^{\text {cond }} \equiv\left[\left\langle\left(E_{s}^{\text {cond }}\right)^{2}\right\rangle-\left\langle E_{s}^{\text {cond }}\right\rangle^{2}\right]^{1 / 2}$ 
and the randomness-induced changes in condensation energies $\mid\left\langle E_{s}^{\text {cond }}\right\rangle-E_{s}^{\text {cond }}($ u.c $) \mid$ were larger for even than for odd grains.

\section{Finite temperature parity effects}

Although finite-temperature studies of the discrete BCS model are not of direct relevance for spectroscopic measurements of the BRT-type (a finite $T$ would simply smear out the discrete spectra, thereby blurring their most interesting features), they are important in their own right for extending our understanding of superconductivity in ultrasmall grains. We hence review several recent finite- $T$ developments below.

To begin, let us note that parity effects are of course not restricted to the $T=0$ limit discussed so far. To be observable [82]- 86 ], they only require the temperature to be smaller than the free energy difference $\delta \mathcal{F} \simeq \tilde{\Delta}-k_{\mathrm{B}} T \ln \left[N_{\text {eff }}(T)\right]$ between an odd and even grain. Here $N_{\text {eff }}(T)$ is the effective number of states available for quasiparticle excitations at temperature $T$, and for $d \ll \tilde{\Delta}$ is given by $N_{\text {eff }}(T)=\sqrt{8 \pi T \tilde{\Delta} / d^{2}}$ [82]. Below the corresponding crossover temperature where $\delta \mathcal{F}=0$, determined by $k_{\mathrm{B}} T_{\mathrm{cr}}^{*}=\tilde{\Delta} \ln \left[N_{\mathrm{eff}}\left(T_{\mathrm{cr}}^{*}\right)\right]$ and roughly equal to $\tilde{\Delta} / \ln \sqrt{8 \pi \tilde{\Delta}^{2} / d^{2}}$, the single unpaired electron begins to matter: it causes a crossover from $e$-periodicty to $2 e$-periodicity in the $I$ - $V$ characteristics of mesoscopic superconducing SET's 82, 83, 84, 85, 86], due to the ground state energy difference $\mathcal{E}_{1 / 2}-\mathcal{E}_{0} \simeq \tilde{\Delta}$. Since $T_{\text {cr }}^{*}$ becomes of order $\tilde{\Delta}$ in nanoscopic grains with $d \simeq \tilde{\Delta}$, parity effects should survive to temperatures as high as the (bulk) superconducting transition temperature $T_{\mathrm{c}}$ itself.

Regrettably, the canonical methods discussed in the preceding sections become impractical at finite temperatures, since the number of states that need to be considered increases rapidly for $T \gtrsim d, \tilde{\Delta}$. On the other hand, g.c. finite- $T$ methods, some of which we review below, are, in principle, inherently unreliable for $d \gtrsim \tilde{\Delta}$. This applies in particular to the simplest of these, parity-projected mean-field theory 79,87 (Sec. 14.1) and certain variational generalizations thereof [21, 22] (Sec. 14.2): they yield the same sharp phase transition as function of temperature for finite systems as for bulk systems, whereas on general grounds no sharp transitions are possible in finite systems. The reason for this problem is that they neglect fluctuations in the order parameter, which become very important in the transition region. The sharp transition is smoothed out once fluctuations are included. A rather efficient way of doing this is the so-called static path approximation (Sec. 14.3). Its use is illustrated in Sec. 14.4 for a calculation of the spin susceptibility, which shows an interesting parity effect that should be measurable in ensembles of ultrasmall grains.

\subsection{Parity-projected mean-field theory}

The simplest finite- $T$ approach that is able to keep track of parity effects is parityprojected mean-field theory, first used in nuclear physics by Tanabe, Tanabe and Mang [102], and, indepedently, introduced to the condensed-matter community by Jankó, Smith and Ambegaokar [79 and Golubev and Zaikin 87]. One projects the g.c. partition function exactly onto a subspace of Fock space containing only even or 


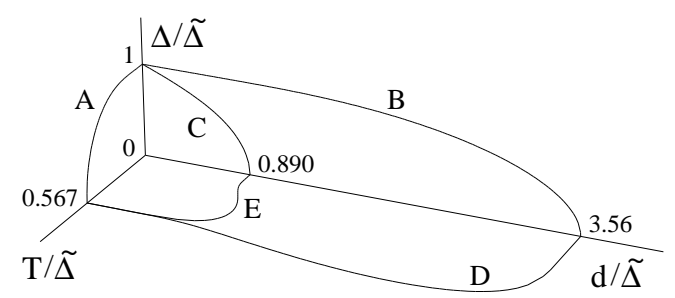

Fig. $18 d$-and $T$-dependence of the pairing parameter $\Delta_{p / 2}(d, T)$, calculated using parityprojected mean-field theory [17]. Curve A gives the bulk gap $\Delta(0, T)$, with $\Delta(0,0) \equiv \tilde{\Delta}$; curves B-E give $\Delta_{p / 2}(d, T) / \bar{\Delta}$ as a function of $d / \tilde{\Delta}$ and $T / \tilde{\Delta}$ for $p=0$ (B, D) and $p=1$ $(\mathrm{C}, \mathrm{E})$. The critical spacings $d_{0}^{\mathrm{BCS}}=3.56 \tilde{\Delta}$ and $d_{1 / 2}^{\mathrm{BCS}}=0.890 \tilde{\Delta}$ given here differ somewhat from those in Fig. 9(a), because the present mean-field approach differs in minor details (via terms that vanish when $d \rightarrow 0$ ) from the variational approach of Sec. 6.2 .

odd $(p=0,1)$ numbers of particles, using the parity-projector $\hat{P}_{p}$ :

$$
Z_{p}^{\mathrm{gc}} \equiv \operatorname{Tr}^{\mathrm{gc}} \hat{P}_{p} e^{-\beta\left(\hat{H}-\mu_{p} \hat{N}\right)}, \quad \hat{P}_{0,1} \equiv \frac{1}{2}\left[1 \pm(-1)^{\hat{N}}\right] .
$$

One then makes the mean-field replacement $b_{j} \rightarrow\left\{b_{j}-\left\langle b_{j}\right\rangle_{p}\right\}+\left\langle b_{j}\right\rangle_{p}$, neglects terms quadratic in the fluctuations represented by \{\} , and diagonalizes $\hat{H}$ in terms of the Bogoljubov quasiparticle operators $\gamma_{j \sigma}$ of Eq. (28). The self-consistency condition $\Delta_{p / 2} \equiv \lambda d \sum_{j}\left\langle b_{j}\right\rangle_{p}$, evaluated in a parity-projected g.c. ensemble according to Eq. (57), leads to a gap equation of the standard form,

$$
\frac{1}{\lambda}=d \sum_{\left|\varepsilon_{j}\right|<\omega_{\mathrm{D}}} \frac{1}{2 E_{j}}\left(1-\sum_{\sigma} f_{p j \sigma}\right), \quad E_{j} \equiv \sqrt{\left(\varepsilon_{j}-\mu\right)^{2}+\left|\Delta_{p / 2}\right|^{2}},
$$

which is parity-dependent, via the occupation function $f_{p j \sigma}=\left\langle\gamma_{j \sigma}^{\dagger} \gamma_{j \sigma}\right\rangle_{p}$ for quasiparticles. Since their number parity is restricted to be $p, f_{p j \sigma}$ differs from the usual Fermi function $f_{j \sigma}^{0}$. The condition $2 n+p=\langle\hat{N}\rangle_{p}$ fixes the chemical potential $\mu$ to lie exactly half-way between the last filled and first empty levels if $p=0$, and exactly on the singly-occupied level if $p=1$, implying $\mu=0$ in both cases [by Eq. (7)].

von Delft et al. 17 applied this approach to the discrete BCS model with uniformlyspaced levels, and solved Eq. (58) for the parity parameter $\Delta_{p / 2}(d, T)$ as function of both level spacing and temperature. Fig. 18 summarizes their results. At zero temperature, $\Delta_{p / 2}(d, 0)$ corresponds to the spin-dependent parity parameters $\Delta_{s=p / 2}$ discussed in Sec. 6.2 [cf. Fig. 9(a)], and drops to zero at a critical level spacing $d_{s}^{\mathrm{BCS}}$. The $\Delta_{p / 2} \rightarrow 0$ limit of Eq. (58) defines the parity-dependent "critical temperature" $T_{c, p}(d)$, which can be viewed as another measure of how rapidly pair-mixing correlations break down as function of level spacing (although ultrasmall grains of course cannot undergo a sharp thermodynamic phase transition, which can only occur if $n \rightarrow \infty)$. In both the even and odd cases, the behavior of $T_{c, P}(d)$ shows direct traces of the parity projection:

In the even case, $T_{c, 0}(d)$ [Fig. 18, curve $\mathrm{D}$ ] is non-monotonic as function of increasing $d$, initially increasing slightly before dropping to zero very rapidly as $d \rightarrow d_{0}^{\mathrm{BCS}}$. The intuitive reason for the initial increase is that the difference between the actual 
and usual quasiparticle occupation functions is $f_{p j \sigma}-f_{j \sigma}^{0}<0$ for an even grain (becoming significant when $d \simeq \tilde{\Delta}$ ), reflecting the fact that exciting quasiparticles two at a time is more difficult than one at a time. Therefore the quasiparticle-induced weakening of pairing correlations with increasing $T$ will set in at slightly higher $T$ if $d \simeq \tilde{\Delta}$.

In the odd case, the critical level spacing $d_{1 / 2}^{\mathrm{BCS}}(T)$ [Fig. 18, curve E] is nonmonotonic as a function of increasing $T$, first increasing to a maximum before beginning to decrease toward $d_{1 / 2}^{\mathrm{BCS}}\left(T_{c}\right)=0$. The intuitive reason for this is that for $0<\Delta_{1 / 2} \ll T, d$, the odd $j=0$ function $f_{p 0 \sigma}(T)$ becomes somewhat smaller than its $T=0$ value of $1 / 2$, because with increasing $T$ some of the probability for finding a quasiparticle in state $j$ "leaks" from $j=0$ to higher states with $j \neq 0$, for which $E_{j}^{-1}<E_{0}^{-1}$ in Eq. (58). Thus, the blocking-of-pair-scattering effect of the odd quasiparticle becomes slightly less dramatic as $T$ is increased, so that $d_{1 / 2}^{\mathrm{BCS}}$ increases slightly.

It should be noted, however, that although the non-monotonicities of $T_{c, 0}(d)$ and $d_{1 / 2}^{\mathrm{BCS}}(T)$ are intuitively plausible within the g.c. framework in which they were derived, their physical significance is doubtful, since they fall in the regime where $d / \Delta_{s} \gtrsim 1$ and the g.c. approach is unreliable, due to its neglect of fluctuations.

\subsection{Variational extensions of BCS theory}

The above-mentioned results of von Delft et al. 117] were reproduced and extended to finite magnetic fields by Balian, Flocard and Vénéroni, using a more general g.c. variational BCS approach [21, 22]. It is designed to optimize the characteristic function $\varphi(\xi) \equiv \ln \operatorname{Tr} \hat{P}_{p} \mathrm{e}^{-\beta(\hat{H}-\mu \hat{N})} \hat{A}(\xi)$, where $\hat{P}_{p}$ is the parity projector of Eq. (57) and $\hat{A}(\xi) \equiv \exp \left(-\sum_{\gamma} \xi_{\gamma} \hat{Q}_{\gamma}\right), \hat{Q}_{\gamma}$ being observables of interest (e.g. the total spin) and $\xi_{\gamma}$ the associated sources (e.g. the magnetic field). This approach goes beyond the usual minimization of the free energy [1], since it optimizes not only thermodynamic quantities but also equilibrium correlation functions, which can be obtained by differentiating $\mathrm{e}^{\varphi(\xi)}$ with respect to $\xi_{\gamma}$. However, its g.c. version also suffers from the drawback of yielding abrupt, spurious phase transitions even though the systems are finite. Presumably this problem would be cured if an exact projection to fixed particle number were incorporated into this approach, but this is technically difficult and has not yet been worked out.

\subsection{Static path approximation}

For finite systems, in contrast to infinite ones, fluctuations of the order parameter about its mean-field value are very important in the critical regime, causing the phase transition to be smeared out; conversely, the spurious sharp transition found in the g.c. approaches above is a direct consequence of the neglect of such fluctuations. A rather successful way of including fluctuations is the so-called static path approximation (SPA), pioneered by Mühlschlegel, Scalapino and Denton [62] and developed by various nuclear theorists 103]- 119], while recently an exact parity projection has also been incorporated [25]-28]. A detailed and general discussion, including a complete list 

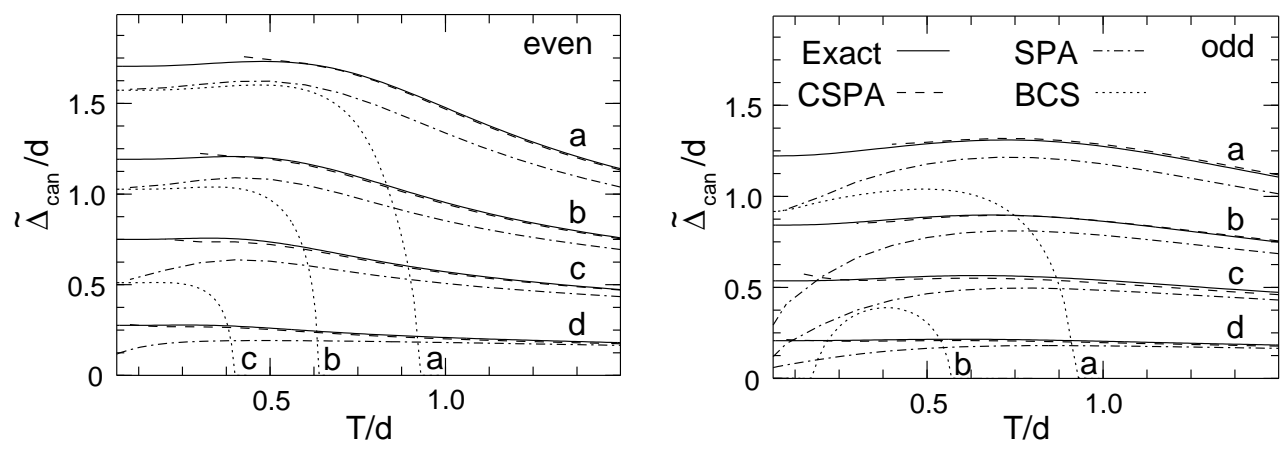

Fig. 19 Temperature dependence of the pairing correlation energy $\tilde{\Delta}_{\text {can }}$ of Eq. (59) [in units of level spacing $d$ ], as calculated in [26] using parity-projected mean-field BCS theory (dotted lines), SPA (dash-dotted), CSPA (dashed) and exact diagonalization (solid lines). A system of 10 equally-spaced, doubly-degenerate, levels was studied, containing 10 (left panel) or 11 (right panel) electrons. $\tilde{\Delta}_{\text {can }}(T)$ is shown at four fixed values of $d / \tilde{\Delta}$ (thus this figure elaborates Fig. 18), namely $0.60,0.91,1.7$, and 15 , labeled by a,b,c and d, respectively (calculated using $\lambda=0.55,0.45,0.35$ and 0.2 ). CSPA data are shown only above the CPSA breakdown, which occurs at $T_{\mathrm{CSPA}}<\tilde{\Delta} / 4$ for the cases considered. The absence of dotted lines for the cases d (even) and c,d (odd) means that for these no nontrivial mean-field BCS solution exists. Of course, $\left(\tilde{\Delta}_{\text {can }}\right)_{\text {exact }}$ is nonzero nevertheless. The abrupt BCS transition is completely smeared out for the SPA, CSPA and exact results, for which the asymptotic decay at $T \gg \tilde{\Delta}$ can be shown 26] to be $\tilde{\Delta}_{\text {can }} \sim \frac{1}{4}\left(\lambda^{3} d / T\right)^{1 / 2}$.

of relevant references, was given very recently by Rossignoli, Canosa and Ring [26]. We therefore confine ourselves below to stating the main strategies of the SPA and illustrating its capabilities by showing its results [Fig. 19] for the quantity

$$
\tilde{\Delta}_{\text {can }}^{2}=(\lambda d)^{2} \sum_{i j}\left[C_{i j}-\left(C_{i j}\right)_{\lambda=0}\right]
$$

$\tilde{\Delta}_{\text {can }}$ is reminiscent ${ }^{j}$ of $\Delta_{\text {can }}$ of Eq. $(22)$, and measures the increase in pairing correlation energy due to a nonzero coupling strength $\lambda$.

One starts by decoupling the quartic interaction $H_{\text {red }}$ of Eq. (6) into a quadratic form using a Hubbard-Stratonovich transformation with a complex auxiliary field $\Delta(\tau)=\Delta^{+}(\tau)+\mathrm{i} \Delta^{-}(\tau)$, with Matsubara-expansion $\Delta^{ \pm}(\tau)=\sum_{n} \Delta_{n}^{ \pm} \mathrm{e}^{\mathrm{i} 2 \pi n \tau / \beta}$ in the interval $\tau \in(0, \beta)$. The parity-projected partition function of Eq. (57) then has the following path integral representation (our notation is deliberately schematic; see 26] for a precise version):

$$
Z_{p} \propto \int \prod_{n} \mathrm{~d} \Delta_{n}^{+} \mathrm{d} \Delta_{n}^{-} \mathcal{Z}_{p}[\Delta], \quad \mathcal{Z}_{p}[\Delta]=\operatorname{Tr}\left\{\hat{P}_{p} \hat{\mathcal{T}} \mathrm{e}^{-\int_{0}^{\beta} \mathrm{d} \tau h[\Delta(\tau)]}\right\},
$$

\footnotetext{
jThe definitions of $\tilde{\Delta}_{\text {can }}$ and $\Delta_{\text {can }}$ differ by terms of order $\left(d / \omega_{H}\right)^{1 / 2}$; for example, when evaluating both using $|\mathrm{BCS}\rangle$ of Eq. (17) and comparing to $\Delta_{\mathrm{gc}}$ of Eq. (18), one finds $\left(\Delta_{\mathrm{can}}\right)_{\mathrm{BCS}}=\Delta_{\mathrm{gc}}=$ $\left(\tilde{\Delta}_{\text {can }}\right)_{\mathrm{BCS}}+\mathcal{O}\left[\left(d / \omega_{D}\right)^{1 / 2}\right]$.
} 


$$
h[\Delta]=\sum_{j \sigma}\left(\varepsilon_{j}-\mu-\lambda d / 2\right) c_{j \sigma}^{\dagger} c_{j \sigma}-\sum_{j}\left(b_{j}^{\dagger} \Delta+\Delta^{*} b_{j}\right)+\frac{|\Delta|^{2}}{\lambda d} .
$$

The path integral can be treated at several levels of sophistication:

(i) In the simplest, one uses a "fixed-phase saddle-point approximation" for the "static" $n=0$ modes and neglects all $n \neq 0$ modes, i.e. one fixes the phase of $\Delta_{0}^{+}+$ $\mathrm{i} \Delta_{0}^{-}=\left|\Delta_{0}\right| \mathrm{e}^{\mathrm{i} \phi_{0}}$ by, say, setting $\phi_{0}=0$, so that $\int \mathrm{d} \Delta_{0}^{+} \mathrm{d} \Delta_{0}^{-}$is replaced by $\int \mathrm{d}\left|\Delta_{0}\right|$, and approximates this integral by its saddle-point value. The saddle-point condition for maximizing $\mathcal{Z}_{p}\left[\left|\Delta_{0}\right|\right]$ then yields the gap equation (58), thus this approach simply reproduces the parity-projected mean-field approach of Sec. 14.1, including its sharp phase transition (Fig. 19, dotted lines).

(ii) The next-best approximation is obtained if one writes $\int \mathrm{d} \Delta_{0}^{+} \mathrm{d} \Delta_{0}^{-}=\int_{0}^{2 \pi} \mathrm{d} \phi_{0}$ $\int_{0}^{\infty}\left|\Delta_{0}\right| \mathrm{d}\left|\Delta_{0}\right|$ and performs the phase integral fully. Remarkably, "liberating" the phase degree of freedom in this way already suffices to smooth out the phase transition 117, 26, even if the $\int \mathrm{d}\left|\Delta_{0}\right|$ integral is again replaced by its saddle-point value, provided that the latter is found by now maximizing $\left|\Delta_{0}\right| \mathcal{Z}_{p}\left[\left|\Delta_{0}\right|\right]$ (i.e. including the factor $\left|\Delta_{0}\right|$ from the integration measure). This yields a modified gap equation with a nontrivial solution for arbitrarily large $T$, i.e. no abrupt transition.

(iii) For finite systems, fluctuations about the saddle become large in critical regions. To obtain an improved description of the latter (Fig. 19, dash-dotted lines), the static path approximation (SPA) [62], [103]-[109 incorporates all static fluctuations exactly, via a (numerical) evaluation of the full integral $\int_{0}^{\infty}\left|\Delta_{0}\right| \mathrm{d}\left|\Delta_{0}\right| \int_{0}^{2 \pi} \mathrm{d} \phi_{0}$ over all "static paths".

(iv) In the so-called correlated static path approximation (CSPA) (also called SPA+RPA), small-amplitude quantum fluctuations around each static path are included too, by performing the remaining $\int \mathrm{d} \Delta_{n \neq 0}^{ \pm}$integrals in the gaussian approximation [110]- 116], [25]-[28]. The CSPA yields qualitatively similar but quantitatively more reliable results (Fig. 19, dashed lines) than the SPA, but breaks down below a temperature $T_{\mathrm{CSPA}}$, below which the fluctuations of the $\Delta_{n \neq 0}^{ \pm}$modes become large at unstable values of $\left|\Delta_{0}\right|$, causing the gaussian approximation to fail.

(v) Finally, in the so-called canonical CSPA one projects the partition function not only to fixed number parity (as done throughout above) but also to fixed particle number, by performing an integration over the chemical potential (before performing any of the $\Delta_{n}^{ \pm}$integrals) 100, 107, 108, 109. However, this too is usually done only in the gaussian approximation (and would produce negligible corrections to the CPSA results for the quantities shown in Fig. 19).

Comparisons with exact diagonalization results 118] (Fig. 19, solid lines) show that in its regime of validity ( $\left.T>T_{\mathrm{CSPA}}\right)$, the CSPA produces results that are qualitatively completely similar and also quantitatively very close to the exact ones, whereas the quantitative agreement is significantly worse if only the SPA is used. Since the CSPA is conceptually simple, well-documented [26] and straightforward to implement, it seems to be the method of choice for not too low temperatures. A possible alternative is a quantum Monte Carlo evaluation of the path integral (60] [120, 121], but the numerics is much more demanding than for the CPSA, while the convergence at low $T$ is in general rather poor, due to the familiar sign problem of Monte Carlo methods.

The development of canonical finite- $T$ methods that remain quantitatively reliable 
for $d \gtrsim \tilde{\Delta}$ and arbitrarily small $T$ is one of the open challenges in this field. It would be very interesting if progress in this direction could be made by exploiting the integrability [94, 95] of the model, using Bethe Ansatz techniques. For the FD regime, another possibility would be to develop a finite- $T$ generalization of the self-consistent RPA approach of Dukelsky and Schuck [37].

\subsection{Re-entrant spin susceptibility}

For grains so small that $d \gg \tilde{\Delta}$, the spectroscopic transport measurements of BRT are not able, in principle, to reliably detect the effect of pairing correlations, since in this regime these cause only small changes to the eigenspectrum of a normal metallic grain, whose spectrum is, however, irregular to begin with. In contrast, thermodynamic quantities do have the potential to measurably reveal the existence of pairing correlations for $d \gg \tilde{\Delta}$. Since very recently parity effects for the spin susceptibility have been observed experimentally for an ensemble of small, normal metallic grains [122], it is an interesting and experimentally relevant question to investigate how pairing correlations affect its behavior in superconducting grains.

This question was worked out in detail by Di Lorenzo et al. 40], whose results are summarized in Fig. 20. The spin susceptibility for an isolated grain is defined as

$$
\chi_{p}(T)=-\left.\frac{\partial^{2} \mathcal{F}_{p}(T, H)}{\partial H^{2}}\right|_{H=0},
$$

where $\mathcal{F}_{p}=-k_{\mathrm{B}} T \ln Z_{p}^{\mathrm{can}}$ is the free energy of a grain with parity $p$ and $Z_{p}^{\mathrm{can}}$ is the canonical partition function.

In the bulk limit [Fig. 20(a)], it is well-known 1223 that the spin susceptibility decreases below its Pauli value $\chi_{\text {Pauli }}=2 \mu_{\mathrm{B}}{ }^{2} / d$ once $T$ drops below the superconducting transition temperature $T_{\mathrm{c}}$, since the electrons tend to bind into Cooper pairs, which are spin singlets and do not contribute to the spin susceptibility. Interestingly, however, the spin susceptibility becomes parity-dependent as $T$ is lowered below the crossover temperature $T_{\mathrm{cr}}^{*}$ mentioned in the opening paragraphs of Sec. 14: In the even case, $\chi_{0}$ exponentially drops to zero for sufficiently small temperatures, $T \ll \max (\tilde{\Delta}, d)$, for reasons that are intuitively obvious in the two limits $\tilde{\Delta} \gg d$ (all electrons bound into Cooper pairs) and $\tilde{\Delta} \ll d$ (no Cooper pairs, but all levels doubly occupied). In contrast, in the odd case $\chi_{1}$ shows a re-entrant behavior, in that it increases as $\mu_{\mathrm{B}}{ }^{2} / T$ for low temperatures, due to a Curie-like contribution from the unpaired odd electron. As a result, $\chi_{1}(T)$ has a minimum somewhat below $T_{\mathrm{cr}}^{*}$, which can be viewed as a "smoking gun" for pairing correlations, since it is absent for odd normal grains. For these, $\chi_{1}(T)$ [long-dashed $\lambda=0$ curve in Fig. 20(b)] also has the Curie-like increase at very low $T$, but lacks the initial pairing-induced decrease as $T$ is reduced below $T_{\mathrm{c}}$.

Remarkably, Di Lorenzo et al. found that this reentrance of $\chi_{1}$ survives also for $d \gtrsim \tilde{\Delta}$ [Fig. 20(b)]: although pairing correlations survive here only as fluctuations, these are evidently sufficiently strong to still significantly reduce $\chi_{1}(T)$ relative to $\chi_{\text {Pauli }}[$ by several percent even for $d / \tilde{\Delta} \simeq 50(!)]$, before the Curie-like increase sets in at low $T$. Di Lorenzo et al. established this result by considering the limits $T \gg d$ and $T \ll 2 d$ analytically, using a static path approximation to capture the crossover numerically, and checking the results for $T \lesssim d$ using Richardson's exact solution 

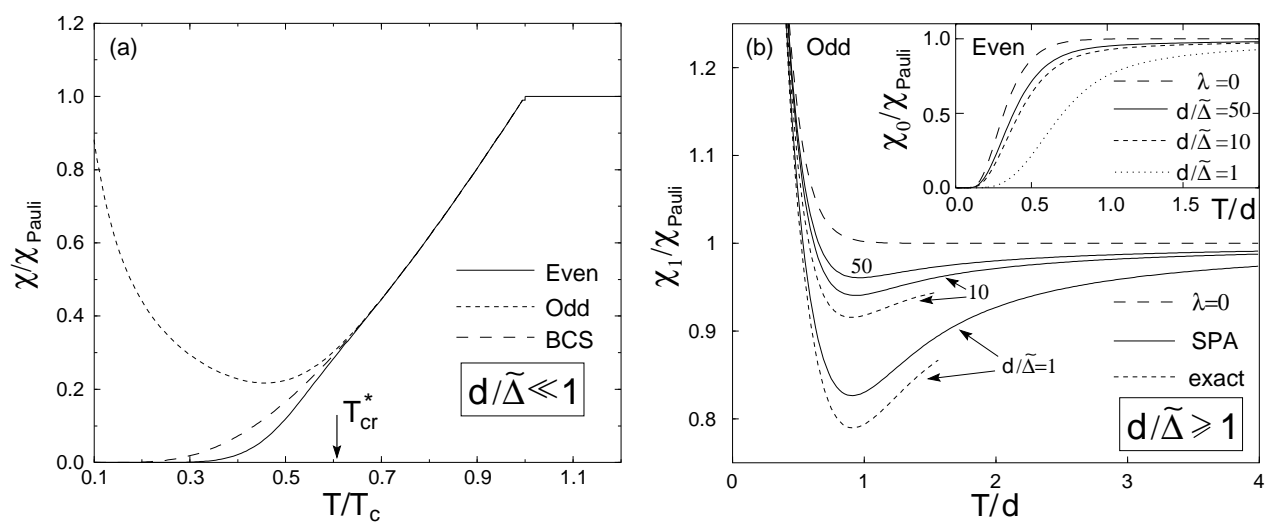

Fig. 20 Spin susceptibility $\chi_{0}\left(\chi_{1}\right)$ of an even (odd) superconducting grain as function of $T$, plotted in units of its bulk high- $T$ value $\chi_{\text {Pauli }}=2 \mu_{\mathrm{B}}{ }^{2} / d$. (a) Bulk limit $(d / \tilde{\Delta} \ll$ $1)$ : the even (solid) and odd (short-dashed) curves were calculated using parity-projected mean-field theory, the long-dashed curve using standard (unprojected) BCS theory. (b) Fluctuation-dominated regime $(d / \tilde{\Delta} \gtrsim 1)$ for $\chi_{1}$ (main figure) and $\chi_{0}$ (inset): All curves were calculated using the static path approximation, except the short-dashed curves in the main figure, calculated using Richardson's exact solution, and the long-dashed curves for the non-interacting case $(\lambda=0)$.

(they considered all eigenstates with excitation energy up to a cutoff $\Lambda \sim 40 d$, for grains with $N \leq 100$ electrons). This check shows that the static path approximation somewhat underestimates the amount of pairing correlations (its minima for $\chi_{1}(T)$ are too shallow), but in general is in good qualitative agreement with the exact results, confirming that it is a useful and qualitatively reliable tool for describing the crossover regime.

\section{Summary and outlook}

The technique of single-electron-tunneling spectroscopy on ultrasmall metallic grains, applied to $\mathrm{Al}$ grains, has proved to be a very fruitful way of probing electron pairing correlations, and the way in which these are modified by level discreteness. It has, in particular, inspired theoretical attempts to quantitatively understand how pairing correlations change during the crossover from the bulk the limit of a few electrons. Let us briefly summarize the main conclusions reached in the preceding sections:

Part I: For largish Al grains, the observation of a distinct spectral gap in even grains and its absence in odd grains is clear evidence for the presence of superconducting pairing correlations. These can be satisfactorily described using the simple discrete BCS model introduced in Sec. 1 . The blocking of some levels by unpaired electrons leads to various measurable parity effects; among these, a pairbreaking-energy parity effect should be observable in experiments of the present kind, provided the grain size can be better controlled. In ultrasmall grains, the effect of a magnetic field on orbital motion is negligible. The dominant mechanism by which a magnetic field destroys 
pairing correlations in ultrasmall grains is Pauli paramagnetism. Decreasing the grain size softens the first-order transition observed for thin films in a parallel field, by reducing the number of spins flipped from being macroscopically large for $d \ll \tilde{\Delta}$ to being of order one for $d \simeq \tilde{\Delta}$. The grand-canonical variational BCS approach fails for $d \gtrsim \tilde{\Delta}$; nevertheless, it yields a useful framework for a qualitative analysis of the experiments, which had $d \lesssim \tilde{\Delta}$.

Part II: The crossover of the behavior of superconducting pairing correlations from the bulk limit $(d \ll \tilde{\Delta})$ to the fluctuation-dominated regime $(d \gg \tilde{\Delta})$ is parity dependent and completely smooth. This remains true for systems with non-uniform rather than uniform level spacings. - Very remarkably, the discrete BCS model has an exact solution, due to Richardson, with which $T=0$ properties can be calculated rather easily. Finite-temperature properties for finite-sized systems can be calculated quite reliably with the correlated static-path approximation (provided $T>T_{\mathrm{CSPA}}$ ). However, the development of canonical finite- $T$ methods that remain quantitatively reliable for $d \gtrsim \tilde{\Delta}$ is still an open problem. - The spin susceptibility $\chi(T)$ of an odd grain shows an interesting reentrant behavior even for $d \gg \tilde{\Delta}$, which might be a way to detect remnants of pairing correlations in the fluctuation-dominated regime.

Finally, we would like to mention two further aspects of pairing correlations in ultrasmall metallic grains that have been discussed in the literature: the effect on the measured excitation spectrum of nonequilibrium excitations [7, 9], and of spin-orbit interactions [11]. These topics have not been included here for lack of space, but have been reviewed in detail in sections 6.2 and 7.6 of Ref. [16].

Prospects for future work:

Experiment: In the current generation of experiments, the grain's actual size and shape cannot be determined very accurately. It would be a great advance if fabrication techniques could be developed to the point that grains can be used which have been custom-made, by chemical techniques, to have well-defined sizes and shapes (e.g. spherical). This would significantly reduce the uncertainties which one presently encounters when estimating characteristic parameters of the grain, such as the single-particle mean level spacing $d$ or the Thouless energy. Moreover, it would allow systematic studies of the dependence of various quantities on grain size or mean level spacing [for example, it would be interesting to try to do this for the pairbreaking energies $\Omega_{e}, \Omega_{o}$ of Fig. 13(b)]. Encouragingly, the feasibility of using chemically-prepared grains in SETs has already been demonstrated several times [89, 90, 91, though the resulting devices have not yet been used for single-electron-tunneling spectroscopy.

Theory: The behavior of superconducting pairing correlations in an individual ultrasmall grain can now be regarded as a subject that is well understood. It would be interesting to try to use the insights that have been gained for a single grain in order to now study systems of several coupled grains: what, for example, is the fate of the Josephson effect between two coupled grains as their sizes are reduced to the point that $d \sim \tilde{\Delta}$ ?

It is a pleasure to thank all my colleagues who actively collaborted in my research on various aspects of the physics of ultrasmall grains: C. Dobler, S. Kleff, J. Kroha, M. Pirmann, G. Schön, W. Thimm, W. Tichy, and A. Zaikin and in particular F. Braun, all in Karlsruhe, and J. Dukelsky, G. Dussel, D. Golubev, D. Ralph, M. Schechter, G. Sierra, and M. Tinkham from other parts of the world. I owe a great deal to D. Ralph for generous amounts of 
enthusiam, expertise and unpublished data on ultrasmall grains, and for critically reading various drafts of the entire review. I also thank F. Braun, P. Brouwer, D. Davidović, M. Greiter, R. Rossignoli, M. Schechter, G. Schön, X. Waintal, for reading and commenting on various parts of the draft. Furthermore I acknowledge helpful and stimulating discussions with O. Agam, I. Aleiner, B. Altshuler, V. Ambegaokar, S. Bahcall, C. Black, Y. Blanter, S. Böcker, P. Brouwer, C. Bruder, T. Costi, M. Deshmuck, F. Evers, G. Falci, R. Fazio, P. Fulde, A. Garg, L. Glazman, D. Golubev, S. Guéron, B. Halperin, B. Janko, J. König, K. Likharev, A. Mastellone, K. Matveev, A. Mirlin, E. Myers, Y. Oreg, T. Pohjola, A. Rosch, J. Siewert, R. Smith, M. Tinkham, F. Wilhelm, N. Wingreen, P. Wölfle, G. Zaránd, F. Zawadowski, and W. Zwerger. This work was supported in part by the Deutsche Forschungsgesellschaft through Sonderforschungsbereich 195. I also acknowledge support from the DFG-Program, "Semiconductor and Metallic Clusters", and from the DAAD-NSF. I thank the editors of Physics Reports and Annalep der Physik for the permission to publish the present review, which is an exerpt of Ref. [16], separately, thereby enabling me to fulfil the habilitation requirements at the University of Karlsruhe.

\section{References}

[1] L. N. Cooper, J. Bardeen, and J. R. Schrieffer, Phys. Rev. 108 (1957) 1175

[2] D. C. Ralph, C. T. Black, and M. Tinkham, Phys. Rev. Lett. 74 (1995) 3241

[3] C. T. Black, D. C. Ralph, and M. Tinkham, Phys. Rev. Lett. 76 (1996) 688

[4] D. C. Ralph, C. T. Black, and M. Tinkham, Physica (Amsterdam) 218B (1996) 258

[5] C. T. Black, PhD thesis, Harvard University, Cambridge 1996

[6] D. C. Ralph, C. T. Black, J. M. Hergenrother, J. G. Lu, and M. Tinkham, p. 447 of Ref. 52

[7] D. C. Ralph, C. T. Black, and M. Tinkham, Phys. Rev. Lett. 78 (1997) 4087

[8] O. Agam, N. S. Wingreen, B. L. Altshuler, D. C. Ralph, and M. Tinkham, Phys. Rev. Lett. 78 (1997) 1956

[9] O. Agam and I. L. Aleiner, Phys. Rev. B 56 (1997) 5759

[10] O. Agam, in Supersymmetry and Trace Formulae: Chaos and Disorder edited by I.V. Lerner, J.P. Keating and D.E. Khmelnitskii, Plenum Press 1999, p. 13

[11] D. G. Salinas, S. Guéron, D. C. Ralph, C. T. Black, and M. Tinkham, Phys. Rev. B 60 (1999) 6137

[12] D. Davidović and M. Tinkham, Phys. Rev. Lett. 83 (1999) 1644

[13] D. Davidović and M. Tinkham, cond-mat/9910396

[14] R. Desmicht and G. Faini, V. Cros, A. Fert, F. Petroff, and A. Vaurés, Appl. Phys. Lett. 72 (1998) 386

[15] S. Guéron and M. M. Deshmukh, E. B. Myers, and D. C. Ralph, Phys. Rev. Lett. 83 (1999) 4148

[16] J. von Delft and D. C. Ralph, to appear in Physics Reports (2001) condmat/0101019.

[17] J. von Delft, A. D. Zaikin, D. S. Golubev, and W. Tichy, Phys. Rev. Lett. 77 (1996) 3189

[18] F. Braun, J. von Delft, D. C. Ralph, and M. Tinkham, Phys. Rev. Lett. 79 (1997) 921

[19] F. Braun and J. von Delft, Phys. Rev. B 59 (1999) 9527

[20] R. A. Smith and V. Ambegaokar, Phys. Rev. Lett. 77 (1996) 4962

[21] R. Balian, H. Flocard, and M. Vénéroni, cond-mat/9802006

[22] R. Balian, H. Flocard, and M. Vénéroni Phvs. Rep. 317 1999) 251

[23] M. C. B $\phi$ nsager and A. H. MacDonald, cond-mat/9812042

[24] K. A. Matveev and A. I. Larkin, Phys. Rev. Lett. 78 (1997) 3749

[25] R. Rossignoli, N. Canosa, and P. Ring, Phys. Rev. Lett. 80 (1998) 1853

[26] R. Rossignoli, N. Canosa, and P. Ring, Ann. Phys. (N.Y.), 275 (1999) 1

[27] R. Rossignoli, J. P. Zagorodny, and N. Canosa, Phys. Lett. A 258 (1999) 188 
[28] R. Rossignoli, private communication (2000)

[29] A. Mastellone, G. Falci and R. Fazio, Phys. Rev. Lett. 80 (1998) 4542

[30] S. D. Berger and B. I. Halperin, Phys. Rev. B 58 (1998) 5213

31] F. Braun and J. von Delft, Phys. Rev. Lett. 81 (1998) 4712

[32] J. Dukelsky and G. Sierra, Phys. Rev. Lett. 83 (1999) 172

[33] J. Dukelsky and G. Sierra, cond-mat/9906166

[34] F. Braun and J. von Delft, in Advances in Solıd State Physics edited by B. Kramer, vol. 39, Vieweg, Braunschweig, 1999, p. 341

[35] G. Sierra, J. Dukelsky, G. G. Dussel, J. von Delft, and F. Braun, Phys. Rev. B 61 (2000) 11890

[36] J. von Delft and F. Braun, cond-mat/9911058, to be published in the Proceedings of the NATO ASI Quantum Mesoscopic Phenomena and Mesoscopic Devices in Microelectronics in Ankara/Antalya, Turkey, June 1999, edited by I.O. Kulik, R. Ellialtioglu, Kluwer Ac. Publishers 2000

[37] J. Dukelsky and P. Schuck, to appear in Phys. Lett. B

[38] G.-S. Tian and L.-H. Tang, Phys. Rev. B 58 (1998) 12333

[39] K. Tanaka and F. Marsiglio, cond-mat/9902173

[40] A. Di Lorenzo, R. Fazio, F. W. Hekking, G. Falci, A. Mastellone, and G. Giaquinta, Phys. Rev. Lett. 84, 550 (2000)

[41] P.W. Anderson, J. Phys. Chem. Solids 11 (1959) 28

[42] F. Braun, PhD thesis, Universität Karlsruhe, Karlsruhe 1999

[43] R. W. Richardson, Phys. Lett. 3 (1963) 277

[44] R. W. Richardson, Phys. Lett. 5 (1963) 82

[45] R. W. Richardson and N. Sherman, Nucl. Phys. 52 (1964) 221

[46] R. W. Richardson, Phys. Lett. 14 (1965) 325

[47] R. W. Richardson, J. Math. Phys. 6 (1965) 1034

[48] R. W. Richardson, Phys. Rev. 141 (1966) 949

[49] R. W. Richardson, Phys. Rev. 144 (1966) 874

[50] R. W. Richardson, Phys. Rev. 159 (1967) 792

[51] R. W. Richardson, J. Math. Phys. 18 (1977) 1802

[52] A series of reviews on various aspects of mesoscopic systems may be found in: Mesoscopic Electron Transport edited by L. L. Sohn, L. P. Kouwenhoven, and G. Schön, NATO ASI Series Vol. 345, Kluwer Ac. Publishers, Dordrecht 1997

[53] A. E. Hanna and M. Tinkham, Phys. Rev. 41 (1991) 5919

[54] D. V. Averin and K. K. Likharev, in Mesoscopic Phenomena in Solids edited by B. L. Altshuler, P. A. Lee, R. A. Webb, Elsevier, New York 1991, p. 169

[55] Single Charge Tunneling edited by H. Grabert and M. H. Devoret, Plenum Press, New York 1992

[56] G. Schön, in Quantum Transport and Dissipation, edited by T. Dittrich, P. Hänggi, G. Ingold, B. Kramer, G. Schön, and W. Zwerger, Wiley-VCH Verlag, 1998, chapter 3

[57] D. V. Averin and A. N. Korotkov, J. Low. Temp. Phys. 80 (1990) 173

[58] M. Tinkham, Introduction to Superconductivity, 2nd Edition, McGraw-Hill, New York 1996

[59] I. Giaever and H. R. Zeller, Phys. Rev. Lett. 20 (1968) 1504

[60] H. R. Zeller and I. Giaever, Phys. Rev. 181 (1969) 789

[61] M. Strongin, R. S. Thompson, O. F. Kammerer, and J. E. Crow, Phys. Rev. B 1 (1970) 1078

62] B. Mühlschlegel, D.J. Scalapino, and R. Denton, Phys. Rev. B 6 (1972) 1767

[63] B. Mühlschlegel, Physica B 203 (1994) 444

[64] A. Kawabata, J. Low Temp. Phys. 38 (1980) 191

[65] A. Kawataba, Surf. Sience, 106 (1981) 358

[66] R. Meservey, P. M. Tedrow, and P. Fulde, Phys. Rev. Lett. 25 (1970) 1270

[67] R. Meservey and P. M. Tedrow, Phys. Rep. 238 (1994) 173

[68] A. Larkin, Sov. Phys. JETP, 21, 153 (1965) [JETP (USSR) 48, 232 (1965)]

[69] V. E. Kravtsov and M. R. Zirnbauer, Phys. Rev. B 46 (1992) 4332

[70] L. I. Glazman, private communication 
[71] K. A. Matveev, L. I. Glazman, and A. I. Larkin, cond-mat/0001431

[72] N. W. Ashcroft and N. D. Mermin, Solid State Physics, Saunders, Philadelphia 1976

[73] J. W. Garland, K. H. Bennemann, and F. M. Mueller, Phys. Rev. Lett. 21 (1968) 1315

[74] V. G. Soloviev, Mat. Fys. Skrif. Kong. Dan. Vid. Selsk. 1 (1961) 1

[75] G. Rickayzen, Theory of Superconductivity, Wiley, New York 1965, chapter 4

[76] P. Ring, P. Schuck, The Nuclear Many-Body Problem, Springer-Verlag, 1980

77] H. J. Lipkin, Ann. Phys. (NY), 9 (1960) 272

[78] B. Mühlschlegel, J. Math. Phys. 3 (1962) 522

[79] B. Jankó, A. Smith, and V. Ambegaokar, Phys. Rev. B 50 (1994) 1152

[80] A. M. Clogston, Phys. Rev. Lett. 9 (1962) 266

81] B. S. Chandrasekhar, Appl. Phys. Lett. 1 (1962) 7

[82] M. T. Tuominen, J. M. Hergenrother, T. S. Tighe, and M. Tinkham, Phys. Rev. Lett. 69 (1992) 1997

[83] M. T. Tuominen, J. M. Hergenrother, T. S. Tighe, and M. Tinkham, Phys. Rev. B 47 (1993) 11599

[84] M. Tinkham, J. M. Hergenrother, and J. G. Lu, Phys. Rev. B 51 (1995) 12649

[85] P. Lafarge, P. Joyez, D. Esteve, C. Urbina, and M.H. Devoret, Phys. Rev. Lett. 70 (1993) 994

[86] T. M. Eiles, J. M. Martinis, and M. H. Devoret, Phys. Rev. Lett. 70, (1993) 1862

[87] D. S. Golubev and A. D.Zaikin, Phys. Lett. A 195, (1994) 380; in Quantum Dynamics of Submicron Structures edited by H. A. Cerdeira et al., Kluwer Ac. Publishers, 1995, p. 473

[88] A. A. Abrikosov, L. P. Gorkov, and I. E. Dzyaloshinksii, Methods of Quantum Field Theory in Statistical Physics, Prentice-Hall, Englewood Cliffs, NJ 1963

[89] D. L. Klein, R. Roth, A. K. L. Lim, A. P. Alivisatos, and P. L. McEuen, Nature 389 (1997) 699

[90] S. Sun and C. B. Murray, J. Appl. Phys. 85 (1999) 4325

[91] A. Bezryadin, C. Dekker, and G. Schmid, Appl. Phys. Lett. 71 (1997) 1273

[92] R. W. Richardson, private communication (1998)

[93] M. Gaudin, Etats et Valeurs Propres de l'Hamiltonien d'Appariement, internal report D.Ph.T/DOC-11/DD, Service de Physique Théorique, Centre d'Etudes Nucléaires de Saclay, BP. no 2 - 92 - GIF-sur-YVETTE 1968

[94] M. C. Cambiaggio, A. M. F. Rivas, and M. Saraceno, Nucl. Phys. A 624 (1997) 157

[95] G. Sierra, Nucl. Phys. B 572 (2000) 517

[96] R. W. Richardson, private communication (1999)

[97] B. F. Bayman, Nucl. Phys. 15 (1960) 33

[98] K. Dietrich, H. J. Mang, and J. H. Pradal, Phys. Rev. 135 (1964) B22

[99] C. W. Ma and J. O. Rasmussen, Phys. Rev. C 16 (1977) 16

[100] C. Essebag and J. L. Egido, Nucl. Phys. A 522 (1993) 205

[101] Moshe Schechter, private communication.

[102] K. Tanabe, S. Tanabe, and H. J. Mang, Nucl. Phys. A 357 (1981) 20

[103] Y. Alhassid and J. Zingman, Phys. Rev. C 30 (1984) 684

[104] Y. Alhassid and B. Bush, Nucl. Phys. A 549 (1992) 43

[105] P. Arve, G. F. Bertsch, B. Lauritzen, and G. Puddu, Ann. Phys. (N.Y.) 183 (1988) 309

[106] B. Lauritzen, P. Arve, and G. F. Bertsch, Phys. Rev. Lett. 61 (1988) 2835

[107] R. Rossignoli, P. Ring, and N. Dang, Phys. Lett. B 297 (1992) 1061

[108] R. Rossignoli, A. Ansari, and P. Ring, Phys. Rev. Lett. B 70 (1993) 9

[109] R. Rossignoli and P. Ring, Ann. Phys. (N.Y.) 235 (1994) 350

[110] G. Puddu, P. F. Bortignon, and R. A. Broglia, Phys. Rev. C 42 (1990) 1830

[111] B. Lauritzen et al. Phys. Lett. B 246 (199) 329

[112] G. Puddu, P. F. Bortignon, and R. A. Broglia, Ann. Phys. (N.Y.), 206 (1991) 409

[113] G. Puddu, Phys. Rev. B 45 (1992) 9882

[114] H. Attias and Y. Alhassid, Nucl. Phys. A 625 (1997) 565

[115] R. Rossignoli and N. Canosa, Phys. Lett. B 394 (1997) 242 
[116] R. Rossignoli and N. Canosa, Phys. Rev. C 56 (1997) 791

[117] R. Rossignoli, N. Canosa, and P. Ring, Nucl. Phys. A 591 (1995) 15

[118] R. Rossignoli, Phys. Rev. C 54 (1996) 1230

[119] R. Rossignoli, N. Canosa, and J. L. Egido, Nucl. Phys. A 605 (1996) 1

[120] G. H. Lang, C. W.Johnson, S. E. Koonin, and W. E. Ormand, Phys. Rev. C 48 (1993) 1518

[121] S.E. Koonin D. J. Dean, and K. Langanke, Phys. Rep. 278 (1997) 1

[122] Y. Volotikin et al. Nature 384 (1996) 621

[123] K. Yosida, Phys. Rev. 110 (1958) 769 